\title{
Epidemiology of paediatric head and facial injuries in Queensland, Australia
}

\author{
Fatima Alsayad \\ College of Public Health, Medical, and Veterinary Sciences, Townsville, Australia \\ Submitted: 11 March 2020 \\ Accepted: 25 April 2020 \\ Arch Med Sci Civil Dis 2020; 5: e58-e91 \\ DOI: https://doi.org/10.5114/amscd.2020.103519 \\ Copyright $\odot 2020$ Termedia \& Banach \\ Corresponding author: \\ Fatima Alsayad \\ College of Public Health \\ Medical and \\ Veterinary Sciences \\ James Cook University \\ 1 James Cook Dr \\ Douglas, 4811 \\ Townsville QLD, Australia \\ E-mail: drfatima2006@yahoo. \\ com
}

\section{Abstract}

Introduction: Head and facial injuries in paediatric patients are a major public health problem in Queensland, Australia, where control, minimisation, or prevention play key roles. Although many studies have been reported, few can be found concerning the epidemiology of paediatric maxillofacial injuries in Queensland, Australia. The aim of this study was to retrospectively assess and report the aetiology, patterns, distribution, and associated factors of head and facial injuries in children in Queensland, Australia.

Material and methods: Data were obtained from Queensland injury Surveillance Unit (QISU). A retrospective study included 10,723 injured children (from less than 1 year old to 12 years old) in the years 2012, 2013, 2014, 2015, and 2016. The analyses involved descriptive statistics and $\chi^{2}$ test.

Results: The number of patients injured varied among age groups. The nature and mechanism of injuries were different among age groups as well. Boys were more prone to maxillofacial injuries compared to females. The highest rate of injuries was in the 1-2-year age group for both genders. Contact with a static object was the main aetiology of maxillofacial injuries during leisure activity (playing). The home was the main site where the injuries occurred.

Conclusions: Preventive measures should be strengthened both indoors and outdoors to reduce the incidence and severity of paediatric injuries in Queensland, Australia. In addition, children in the growing phase should be monitored periodically. Furthermore, increasing awareness can be attained by increasing safety education.

Key words: injuries, children, paediatric, maxillofacial injuries, risk factors.

\section{Introduction}

Head and facial injuries represent a serious public health concern worldwide, according to the World Health Organisation (WHO) [1]. In Australia and other developed countries, paediatric head and facial injuries are common causes of paediatric morbidity and mortality, despite advancements in child safety [2].

Head and face injuries, which are a common presentation to every general practice and every hospital emergency department in Queensland, Australia [3], represent a group of injures on the head and face commonly resulting from various insults to the head and face by external force, classified as intentional and unintentional injuries [4]. 
These injuries, comprising hard tissue as well as soft tissue injuries, exhibit different patterns and clinical features based on the severity and mechanism of the injury [5]. Additionally, they occur as isolated injuries or in combination with other severe injuries, including spinal, brain, and upper and lower body injuries, depending also on the aetiology and severity of the injuries [3].

Injuries to these regions may be particularly disabling because they are regions of specialised functions such as vision, hearing, olfaction, respiration, mastication, and speech [6]. Additionally, important vascular and neural structures that are closely associated are present in these regions and might be affected by such injuries. For example, damage to the central nervous system may occur, and injuries in this region can result in serious dysfunction and might cause disability [6]. Also, the psychological impact of scars or disfigurement in the face after recovery may add to the level of resulting morbidity [7].

Moreover, severe head and face injuries can expose patients to life-threatening complications such as airway obstruction and haemorrhagic shock or intracranial and brain injuries [8].

The incidence and an aetiology of maxillofacial injuries varies from one country to another, and even from one region to another in the same country, depending on prevailing socio-economic, cultural environmental factors, lifestyle, and legislative measures [9]. Additionally, there are other factors (injury risk factors) affecting the incidence and the outcome of head and facial injuries. For example, the type and part of place where the child is injured, the activity being undertaken the when the injury occurred, the temporal influence factor, the severity of the injuries, the nature of the injuries, and the management of the patient (admission status). Thus, an understanding of these factors can aid in establishing clinical and research priorities for effective treatment of these injuries. Also, such factors can help to guide the development of new methods of injury prevention and preventative measures [10].

However, head and face injuries in paediatric patients are different from those in adults, although both children and adults are subjected to similar types of injuries [5]. Children are more susceptible to craniofacial trauma due to their greater cranial mass-to-body ratio [11]. Additionally, the head and face of children are not fully formed, and future growth will be a factor in how the child heals and recovers. There are certain types of injuries that may cause a delay in the growth or further complicate recovery [12]. Furthermore, difficult cases of such injuries require physicians and surgeons with great skill to make a repair that will grow with the child [12].
Moreover, the management of paediatric patients with head and facial injuries should take into consideration the differences in anatomy and physiology between children and adults, the presence of concomitant injuries, the particular stage in growth and development (anatomic, physiology, and psychology), the specific injuries, and anatomic sites that the injuries affect [12, 13]. Further, clear diagnosis and early treatment are keys to prevent complications and undesirable results [5].

Paediatric head and face injuries are not only a leading cause of morbidity and mortality, but also have negative effects on quality of life, functional status, and social functioning. Furthermore, they have a range of economic impacts, including health care costs, productivity, and insurability [14].

Thus, epidemiological analysis of paediatric head and face injuries is important to identify the burden of such injuries and to help develop more efficient ways to plan resource allocation, to deliver care, and guide preventive measures [14, 15]. Furthermore, knowing the epidemiology profile of children with head and face injuries allows us to monitor and control certain risk factors that would prevent or lessen the incidence of such injuries [16-18].

Although many overseas studies have been reported, there are few reports detailing the causes and incidences of paediatric head and face injuries in Queensland, Australia. This retrospective report presents a study investigating the aetiology and incidence of paediatric patients with head and face injuries in Queensland, Australia in a period of time (2012, 2013, 2014, 2015, and 2016).

\section{Material and methods}

Data were accessed from the Queensland Injury Surveillance Unit (QISU) database, which collects and collates injury data from a number of Queensland public hospital emergency departments (EDs).

Data were included for children aged from birth to 12 years, with year of presentation 2012-2016 inclusive. To extract information about children with head and facial injuries, the following terms were used: BODY_REGIONS_CODE: 22 (body location not required) or 02 (Face (excludes eye)) or 01 (Head) or 21 (Multiple injuries involving more than one bodily location) and Like "*face" Or Like “* lip *” Or Like “* lip*” Or Like “*lip *” Or Like "*nose*" Or Like "*eye*" Or Like "*mandibular"” Or Like “*VERMIL" Or Like “*nare*”.

\section{Statistical analysis}

Data from QISU had already been cleaned no duplicate entries were present, and all entries were sufficiently complete. 
Values within variables were coded and labelled as necessary, using the information contained within the dataset and with reference to National Data Standards for Injury Surveillance, Version 2.1 (Ref).

Some variables were collapsed to reduce the number of categories to allow meaningful summarisation and for purposes of statistical analysis. Decisions about collapsing were based on combining logically similar categories, using information contained within the dataset, the National Data Standards for Injury Surveillance, Version 2.1 (Ref), and information about ICD coding from the World Health Organisation ICD-10 Online Version (Ref).

The patients were grouped into five age bands: $<1,1-2,3-4,5-9$, and $10-12$ years.

The time of presentation was grouped into 3-hour periods, except for 9 p.m.-6 a.m., which was analysed as one block due to a lower number of presentations.

Some values were coded as 'missing' for the purpose of conducting statistical tests. For example, indigenous status coded as 'not stated/unknown' was recoded as 'missing' for the purpose of statistical analyses, leaving two collapsed categories of 'not indigenous' and indigenous and/or Torres Strait Islander'.

Descriptive and analytical analyses were conducted using Statistical Package for Social Sciences (SPSS) version 25.

The majority of variables were categorical; therefore $\chi^{2}$ tests were performed when investigating associations between two variables. The assumptions for a valid $\chi^{2}$ test were considered to be fulfilled when the following criteria were met: - Assumption 1: No more than $20 \%$ of cells can have an expected count of less than 5 , and

- Assumption 2: The minimum expected count is 1 or greater.

If assumptions were not fulfilled, the test was not conducted. No Exact tests were performed. A $\chi^{2}$ test for trend was used with cross-tabulations involving age groups. The $\chi^{2}$ tests for goodness of fit were also conducted to compare time data on time of day, day of week, month of year, season of year, and calendar year against a uniform distribution. When analysing calendar year, month of year, and season of year, data from 2016 were excluded because only 6 months of data were available for this year (Table I).

\section{Results}

\section{Sample characteristics}

\section{Demographic characteristics}

\section{Gender}

There were a total of 10,723 head and facial injury cases over the duration of the study. Males comprised $62.5 \%$ of cases $(n=6702)$ (Table II), yielding a male-to-female ratio of $1.7: 1$.

\section{Age}

Mean age was $4.34 \pm 3.38$ years. Figure 1 shows the distribution of age in years. The most commonly presenting age was 1-year-olds ( $n=1976$,

Table I. Variables analysed in this study

\begin{tabular}{|c|c|}
\hline Age group & Infant ( 0 years), $1-2,3-4,5-9$, and $10-12$ years age groups \\
\hline Indigenous status & Not indigenous; indigenous (Aboriginal and/or Torres Strait Islander) \\
\hline Country & Country of birth \\
\hline Language & Preferred language \\
\hline Time & Time of day of presentation to emergency department on which the injury was sustained \\
\hline Day & Day of week of presentation to emergency department \\
\hline Weekend and weekdays & Weekend and weekdays of presentation to emergency department \\
\hline Season & Season of presentation to emergency department \\
\hline Calendar of year & Year of presentation to emergency department $(2012,2013,2014,2015$, and 2016) \\
\hline Triage & Triage score \\
\hline Mechanism of injury & The way the injury was sustained (aetiology) \\
\hline ICD of injury & International classification of diseases applied for injury \\
\hline Mode of separation & Management of patient following emergency department attendance (Admission status) \\
\hline Nature of injuries & Type of injury sustained \\
\hline Body regions & The region or part of the body where the injury is located \\
\hline Intent & The role of the patient in case of injury \\
\hline Type of place & Type of place where the child was injured (first tier classification) \\
\hline Part of place & Part of place where the child was injured (second tier classification to 'Type of place') \\
\hline Activity & The activity the injured child was engaged in when the injury occurred \\
\hline
\end{tabular}


Table II. Demographic characteristics

\begin{tabular}{|c|c|c|c|}
\hline \multicolumn{2}{|c|}{ Demographic characteristics } & \multirow{2}{*}{$\begin{array}{c}\text { Number } \\
10723\end{array}$} & \multirow{2}{*}{$\begin{array}{c}\% \\
100\end{array}$} \\
\hline Total number & & & \\
\hline \multirow[t]{2}{*}{ Gender: } & Male & 6702 & 62.5 \\
\hline & Female & 4021 & 37.5 \\
\hline \multirow[t]{5}{*}{ Age groups: } & Infants (0 years) & 695 & 6.5 \\
\hline & $1-2$ years & 3511 & 32.7 \\
\hline & $3-4$ years & 2201 & 20.5 \\
\hline & $5-9$ years & 3120 & 29.1 \\
\hline & $10-12$ years & 1196 & 11.2 \\
\hline \multirow[t]{3}{*}{ Indigenous status: } & Not Indigenous & 9832 & 91.7 \\
\hline & Aboriginal and/or TSI & 777 & 7.2 \\
\hline & $\begin{array}{c}\text { Indigenous status not } \\
\text { stated/unknown }\end{array}$ & 114 & 1.1 \\
\hline \multirow[t]{7}{*}{ Country of birth: } & Australia & 9956 & 92.8 \\
\hline & Not stated & 259 & 2.4 \\
\hline & New Zealand & 97 & 0.9 \\
\hline & England & 72 & 0.7 \\
\hline & India & 39 & 0.4 \\
\hline & United States of America & 35 & 0.3 \\
\hline & Other countries & 265 & 2.5 \\
\hline \multirow[t]{3}{*}{ Spoken language } & English only & 10,493 & 97.9 \\
\hline & $\begin{array}{l}\text { Language(s) in addition to } \\
\text { English }\end{array}$ & 169 & 1.5 \\
\hline & Not stated & 61 & 0.5 \\
\hline
\end{tabular}

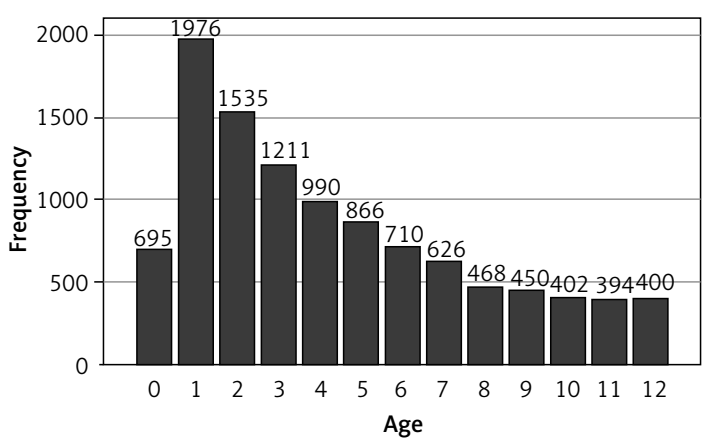

Figure 1. Distribution of ages in years

$18.4 \%$ ), followed by 2 -year-olds ( $n=1535,14.3 \%$ ) and 3 -year-olds ( $n=1211,11.3 \%$ ), with the number of cases decreasing with each year of age until 10-12-years-olds (each approximately 400 cases and $3.7 \%)$. There were 695 (6.5\%) children who presented as infants less than 1 year old.

The majority of cases were non-indigenous 9832 (91.7\%), while 777 (7.2\%) identified as Aboriginal or Torres Strait Islander or both, and 114 (1.1\%) had unknown indigenous status (Table II). Of the 10,609 children reported, 9832 (91.7\%) were non-indigenous, and 777 (7.2\%) identified as Aboriginal or Torres Strait Islander or both. Of the 10,723 patients 114 (1.1\%) were stated as missing data (indigenous status not stated/unknown), and only 10,609 (98.9\%) children were valid.
Additionally, most patients (9956, 92.8\%) were born in Australia. The next most frequently represented countries are presented in Table II. For $97.9 \%$ of cases ( $n=10,493)$, English was the only language spoken, while $1.5 \%(n=169)$ spoke additional languages.

\section{Injury characteristics}

Mechanism of injury

Figure 2 shows the mechanism of injury for children who sustained head and face injuries. Of the 16 categories, contact with static objects was the leading aetiology, occurring in 2238 (20.9\%) patients. Cutting or tearing was the second most frequent aetiology, affecting 1359 (12.7\%) patients, and other falls (height not specified) was the third most frequent aetiology (1085, 10.1\%). Other causes of injury included fall by stumbling, tripping on the same level (1056, 9.8\%), fall/jump from a lesser height (less than $1 \mathrm{~m})(980,9.1 \%)$, contact with a moving object $(956,8.9 \%)$, contact with a person (895, 8.3\%), miscellaneous and unspecified mechanism of injury $(469,4.4 \%)$, bite, sting by animal, human, or insect (380, 3.5\%), fall on or from stairs (292, 2.7\%), other/unspecified contact $(231,2.2 \%)$, burns $(201,1.9 \%)$, contact with animal (180, 1.7\%), fall/jump from a greater height (more than one metre) $(139,1.8 \%)$, and 
contact, splash, or spill $(46,0.4 \%)$. In total, falls of some kind represented $33.63 \%$ of head and facial injuries in children aged $0-12$ years.

Injury severity (Triage score)

The triage score is the urgency of the person's need for medical and nursing care. The majority of the injury cases in this study needed semi-urgent intervention within $60 \mathrm{~min}(n=7315,68.2 \%)$ followed by urgent triage within $30 \mathrm{~min}(n=2284,21.3 \%)$. Only $5.5 \%$ of cases required emergency treatment (< $10 \mathrm{~min} ; n=593)$, and $4.9 \%$ required non-urgent treatment $(<120 \mathrm{~min} ; n=530)$ (Table III).

Intent

Nearly all injuries were unintentional ( $n=$ 10542, 98.3\%). A total of 101 (0.9\%) injuries were of unclassified or undetermined intent, and $80(0.7 \%)$ were due to assault/harm by another person, as shown in Table III.

Nature of injuries

The most frequent injuries were open wound (excludes eye) (4527, 42.25\%), followed by superficial (incl. bruise; excl. eye) (3303, 30.8\%). The third most common injury was eye injury (excl. foreign body in external eye) (1790, 16.7\%).

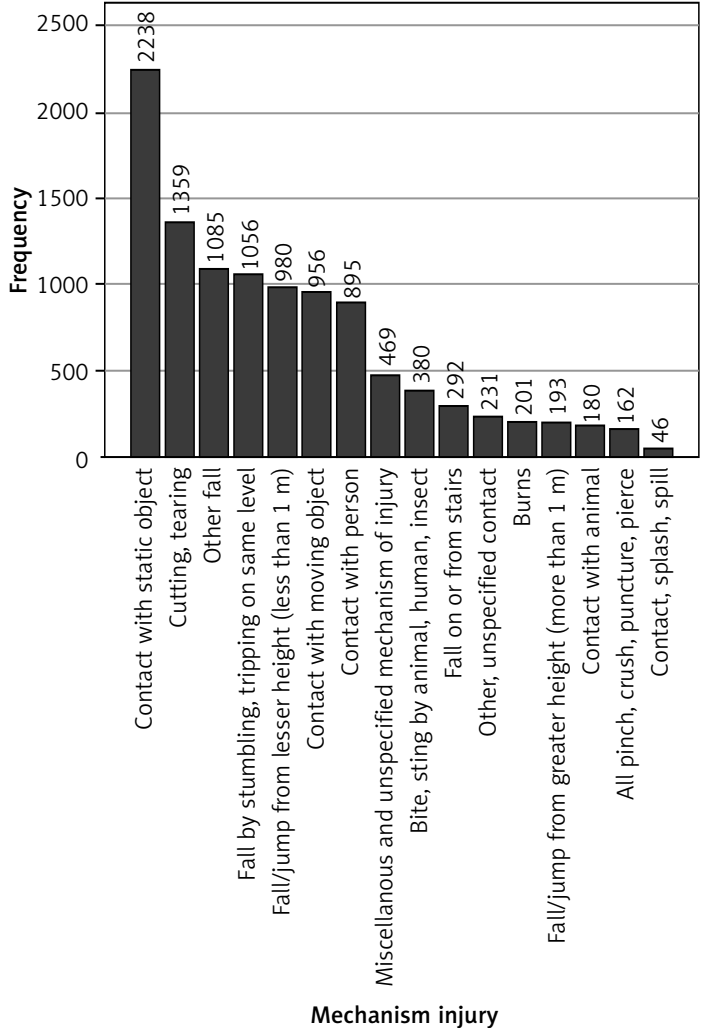

Figure 2. Mechanism of injury

Table III. Injury characteristics

\begin{tabular}{|c|c|c|}
\hline \multirow[t]{4}{*}{ Triage (severity)* } & Semi urgent (60 min) & $7315,68.2 \%$ \\
\hline & Urgent (30 min) & $2284,21.3 \%$ \\
\hline & Emergency & $593,5.5 \%$ \\
\hline & Non urgent (120 min) & $530,4.9 \%$ \\
\hline \multirow[t]{3}{*}{ Intent } & Accident; injury was not intended & $10542,98.3 \%$ \\
\hline & Event of unclassified or undetermined intent & $101,0.9 \%$ \\
\hline & Assault/harm by another person & $80,0.7 \%$ \\
\hline \multirow{9}{*}{ Nature of injury } & Open wound (excludes eye) & $4527,42.2 \%$ \\
\hline & Superficial (incl. bruise; excl. eye) & $3303,30.8 \%$ \\
\hline & Eye injury (excl. foreign body in external eye; includes burns) & $1790,16.7 \%$ \\
\hline & Miscellaneous, esp. foreign body & $313,2.9 \%$ \\
\hline & Fracture and dislocation (excludes tooth) & $237,2.2 \%$ \\
\hline & Dental injury (incl. fractured tooth) & $227,2.1 \%$ \\
\hline & Burn or corrosion (excl. eye) & $176,1.6 \%$ \\
\hline & Intracranial injury (includes concussion) & $94,0.9 \%$ \\
\hline & Effect of venom; any insect bite & $56,0.5 \%$ \\
\hline \multirow[t]{3}{*}{ Mode of separation } & ED service event completed discharged & $8904,83.0 \%$ \\
\hline & Admitted to same or other hospital & $1661,15.5 \%$ \\
\hline & Did not wait/left after treatment commenced & $158,1.5 \%$ \\
\hline \multirow[t]{4}{*}{ Body regions injured } & Face (excludes eye) & $4391,40.9 \%$ \\
\hline & Head (excludes face) & $4082,38.1 \%$ \\
\hline & Body location not required & $2082,19,4 \%$ \\
\hline & Multiple injuries (involving more than one body location) & $168,1.6 \%$ \\
\hline
\end{tabular}

${ }^{\star}$ Total valid responses for triage score, $n=10,722$. 
Fracture (excludes tooth) and dental injury (incl. fractured tooth) amounted to 237 (2.2\%) and 227 (2.1\%), respectively, of the total number (Table III).

The mode of separation describes how the episode of treatment ended. Most cases were treated in the ED and were discharged home $(n=8904$, 83.0\%). Approximately 1 in 6 cases $(n=1661$, $15.5 \%)$ were admitted to the same or another hospital. Additionally, 158 (1.5\%) patients did not wait or left after treatment commenced.

Body region injured

Because this study examines paediatric head and facial injury, the majority of the injuries were coded as involving the face (4391, 40.9\%) and head (excluding the face) (4082, 38.1\%). Eye injuries are not coded within the 'face' code, instead falling within 'body location not required' (2082, $19.4 \%$ ). The 'body location not required' code is a grouping that includes injuries for which the injury location is evident from the nature of the injury, such as eye injury or concussion. Also included in the study were 168 (1.6\%) cases with multiple injuries (involving more than one bodily location).

\section{Distribution of injury by time, month,} season, year, time of day, and day of week

Table IV shows the temporal characteristics of head and face injuries. Injuries most frequently occurred in the three-hour time period between 3 and 6 p.m. ( $n=3016,28.1 \%)$, and least frequently between 6 and 9 a.m. ( $n=765,7.1 \%)$.

The frequency of presentation of head and facial injuries by day of week varied significantly from a uniform distribution $\left(\chi^{2}=201.67, \mathrm{~d} f=6\right.$, $p<0.001)$. The greatest number of cases present- ed on Sundays ( $n=1925,18.0 \%$ ), and the least on Tuesdays ( $n=1289,12.0 \%)$. Additionally, significantly more injuries on a per-day basis occurred on weekend days ( $n=3683$, equivalent to $17.2 \%$ per day) than weekdays ( $n=7040$, equivalent to $13.1 \%$ per day; $\left.\chi^{2}=175.08, \mathrm{~d} f=1, p<0.001\right)$.

\section{Calendar year, month, and season}

Of the four complete calendar years in the study period, there were significance differences in the number of presentations per year, with the greatest number of cases occurring in the year $2015(n=2529,26.9 \%)$ and the least in $2012(n=$ $2099,22.3 \%)\left(\chi^{2}=52.46, \mathrm{~d} f=3, p<0.001\right)$. Also, when analysed with the complete years only, the number of presentations per month differed significantly $\left(\chi^{2}=31.87, \mathrm{~d} f=11, p<0.001\right)$, with the maximum in August $(852,9.0 \%)$ and the minimum in February $(665,7.1 \%)$ (Table V).

There was no significant difference in presentations by season, when analysed with complete years only $\left(\chi^{2}=2.75, \mathrm{~d} f=3, p=0.432\right)$.

\section{Event characteristics}

As shown in Table VI, the event characteristics included the activity the child was engaged in when the injury occurred, and the type and part of place where the injury occurred.

\section{Sub-analyses of injury and event characteristics}

\section{Injury and event characteristics by gender}

The proportion of males and females varied significantly with age group $\left(\chi^{2}=32.64, \mathrm{~d} f=4\right.$, $p<0.001)$. Males were over-represented in each

Table IV. Head and facial Injuries by time, day, season, and year

\begin{tabular}{|c|c|c|c|}
\hline Injury characteristics & & Number & $\%$ \\
\hline \multirow{6}{*}{$\begin{array}{l}\text { Time of injury: } \\
p<0.001\end{array}$} & 6-9 a.m. & 765 & 7.1 \\
\hline & 9 a.m. - midday & 1794 & 16.7 \\
\hline & Midday - 3 p.m. & 1902 & 17.7 \\
\hline & 3-6 p.m. & 3016 & 28.1 \\
\hline & 6-9 p.m. & 2283 & 21.3 \\
\hline & 9 p.m. -6 a.m. & 963 & 9 \\
\hline \multirow{7}{*}{$\begin{array}{l}\text { Days of week: } \\
p<0.001\end{array}$} & Mon & 1398 & 13.0 \\
\hline & Tue & 1289 & 12.0 \\
\hline & Wed & 1428 & 13.3 \\
\hline & Thu & 1390 & 13.0 \\
\hline & Fri & 1535 & 14.3 \\
\hline & Sat & 1758 & 16.4 \\
\hline & Sun & 1925 & 18.0 \\
\hline \multirow{2}{*}{$\begin{array}{l}\text { Weekdays and weekends: } \\
p<0.001\end{array}$} & Weekdays & 7040 & 65.7 \\
\hline & Weekend & 3683 & 34.3 \\
\hline
\end{tabular}


Table V. Head and facial injuries by month and season (analysed for 2012-2015, calendar years with complete data)

\begin{tabular}{|c|c|c|}
\hline Calendar year & Number & $\%$ \\
\hline 2012 (12 months) & 2099 & 22.3 \\
\hline 2013 (12 months) & 2283 & 24.2 \\
\hline 2014 (12 months) & 2505 & 26.6 \\
\hline 2015 (12 months) & 2529 & 26.9 \\
\hline \multicolumn{3}{|l|}{ Months: } \\
\hline January & 802 & 8.5 \\
\hline February & 665 & 7.1 \\
\hline March & 787 & 8.4 \\
\hline April & 754 & 8.0 \\
\hline May & 790 & 8.4 \\
\hline June & 757 & 8.0 \\
\hline July & 789 & 8.4 \\
\hline August & 852 & 9.0 \\
\hline September & 783 & 8.3 \\
\hline October & 826 & 8.8 \\
\hline November & 778 & 8.3 \\
\hline December & 833 & 8.8 \\
\hline Total & 9416 & 100 \\
\hline \multicolumn{3}{|l|}{ Seasons: } \\
\hline Spring (Sept-Nov) & 2387 & 25.4 \\
\hline Summer (Dec-Feb) & 2300 & 24.4 \\
\hline Autumn (March-May) & 2331 & 24.8 \\
\hline Winter (June-Aug) & 2398 & 25.5 \\
\hline Total & 9416 & 100 \\
\hline
\end{tabular}

age group, but the over-representation was lowest in the first year of life ( $57.8 \%$ male, $42.2 \%$ female). The gender disparity in each age group increased with age, as shown in Table VII.

\section{Mechanism of injury by gender}

There was a significant association between gender and mechanism of injury $\left(\chi^{2}=79.036\right.$, $\mathrm{d} f=15, p<0.001)$. Table VIII shows that males were particular overrepresented in the mechanism of 'all pinch, crush, puncture, pierce' (71.0\%); 'contact with person' (68.7\%); and 'cutting, tearing' (63.6\%). Females were overrepresented in 'contact with animal' (53.3\%); and less underrepresented in 'bite or sting by animal, human, insect' (47.1\%); and 'fall on or from stairs' (46.9\%).

\section{Activity by gender}

There was a significant association between gender and activity engaged by children at the time of injury $\left(\chi^{2}=187.29, \mathrm{~d} f=18, p<0.001\right)$. Table IX shows that males were particularly overrepresented in the activity of 'football, rugby, soccer' (94.4\%); 'roller blading, skateboarding' (76\%); and 'bicycling' (73\%). Females were overrepresented in 'horse riding' (92.9\%); 'gymnastics \& martial arts' (57.5\%); and 'basketball, netball' (54\%).

\section{Nature of injury by gender}

There was a significant association between nature of injury and gender $\left(\chi^{2}=28.43, \mathrm{~d} f=8, p<\right.$ $0.001)$. Table $X$ shows that males were particularly overrepresented in 'dental injury (incl. fractured tooth)' (65.2\%), 'eye injury (excl. foreign body in external eye)' (65\%), and 'open wound (excludes eye)' (63.9\%). Females were less underrepresented in 'effect of venom; any insect bite' (50\%), 'superficial' (40.3\%), and 'intracranial injury (includes concussion)' (37.2\%).

\section{Type of place by gender}

There was a significant association between nature of injury and gender $\left(\chi^{2}=94.92, \mathrm{~d} f=13\right.$, $p<0.001$ ). Table XI shows that males were particularly overrepresented in childcare, preschool centre (68.2\%); bush, remote or undeveloped place $(66.7 \%)$; and hospital, medical clinic, or other health service (66.7\%), where injuries occurred. Females were overrepresented in farm (excl. farmhouse) (50.0\%); other and unspecified sports area (43.6\%); and trade or service area, incl. shopping centre, restaurant $(41.5 \%)$ as the type of place where injuries occurred within children.

\section{Other gender associations:}

There were no associations between gender and triage code $(p=0.110)$, mode of separation $(p=0.539)$, body region $(p=0.073)$, or intent (0.134).

\section{Injury and event characteristics by age group}

In all age groups, the vast majority of injuries were considered unintentional (98.3\%), as shown in Table XII. Also, consistently across all age groups, most cases were triaged as semi-urgent (requiring treatment within $60 \mathrm{~min})(68.2 \%)$, and in most cases the ED service event was completed and the patient was discharged home (83.0\%) (Table XII). There were significant associations between age group and intent $\left(\chi^{2}=126.67, \mathrm{~d} f=8\right.$, $p<0.001)$, severity $\left(\chi^{2}=236.63, \mathrm{~d} f=12, p<0.001\right)$, body region $\left(\chi^{2}=582.44, \mathrm{~d} f=12, p<0.001\right)$, and outcome of visit $\left(\chi^{2}=36.99, \mathrm{~d} f=8, p=0.003\right)$.

For example, 10-12-year-olds were more likely to have an injury through assault/harm by another person (3.2\%), while infants were more likely to have an event of unclassified or undetermined intent (1.6\%). 10-12-year-olds were more likely to require emergency $(10.1 \%)$ or urgent $(30.4 \%)$ treatment, while infants were less likely to be classified as requiring non-urgent treatment (3.5\%). 10-12-year-olds were most likely to have their ED event completed and be discharged (85.6\%), while infants were most like- 
Table VI. Event characteristics

\begin{tabular}{|c|c|c|}
\hline $\begin{array}{c}\text { Activity } \\
(10723,100 \%)\end{array}$ & $\begin{array}{l}\text { Type of place } \\
(10723,100 \%)\end{array}$ & $\begin{array}{l}\text { Part of place } \\
(10723,100 \%)\end{array}$ \\
\hline Leisure activity: 4815, 44.9\% & Home: 6986, 65.1\%, & $\begin{array}{l}\text { Unspecified part of place: } 4099 \text {, } \\
38.2 \%\end{array}$ \\
\hline $\begin{array}{c}\text { Resting, sleeping, eating, other: } \\
1583,14.8 \%\end{array}$ & Unspecified place: $834,7.8 \%$. & $\begin{array}{c}\text { Garden, park, national park } 794, \\
7.4 \%\end{array}$ \\
\hline Unspecified activity: 1452, 13.5\% & $\begin{array}{c}\text { Schools primary or secondary: } \\
741 \text { cases } 6.9 \%\end{array}$ & Other interior $784,7.3 \%$ \\
\hline Being nursed or cared for: $988,9.2 \%$ & Childcare/preschool: $428,4.0 \%$ & Bedroom: $783,7.3 \%$ \\
\hline Other specified activity: $909,8.5 \%$ & Public parks: $372,3.5 \%$ & Living, family, rumpus room: $747,7 \%$ \\
\hline $\begin{array}{c}\text { Engaged in formal education activity } \\
\text { as a student: } 507,4.7 \%\end{array}$ & Oval/field/pitch: $277,2.6 \%$ & Other exterior: $576,5.4 \%$ \\
\hline Sports activity: $455,4.2 \%$ & Street/highway: $236,2.2 \%$ & Bathroom: $354,3.3 \%$ \\
\hline \multirow[t]{19}{*}{$\begin{array}{l}\text { Other type of work incl. unpaid } \\
\text { housework: } 14,0.1 \%\end{array}$} & $\begin{array}{c}\text { Recreation areas, including } \\
\text { amusement park, aquatic recreation } \\
\text { centre: } 229,2.1 \%\end{array}$ & $\begin{array}{l}\text { Playground with or without play } \\
\text { equipment: } 534,5 \%\end{array}$ \\
\hline & $\begin{array}{c}\text { Miscellaneous specified place: } \\
229,2.1 \%\end{array}$ & Stairs: $335,3.1 \%$ \\
\hline & $\begin{array}{c}\text { Trade or service area, including } \\
\text { shopping centre and restaurant: } \\
200,1.9 \%\end{array}$ & Kitchen: $304,2.8 \%$ \\
\hline & $\begin{array}{l}\text { Other and unspecified sports areas: } \\
\qquad 78,0.7 \%\end{array}$ & Oval/field: 278, 2.6 \\
\hline & Farm excluding farmhouse: $44,0.4 \%$ & Swimming pool: $180,1.7 \%$ \\
\hline & $\begin{array}{l}\text { Bush, remote or undeveloped place: } \\
\qquad 2,0.4 \%\end{array}$ & Car park/driveway: $164,1.5 \%$ \\
\hline & $\begin{array}{l}\text { Hospital, medical clinic or other } \\
\text { health service: } 27,0.3 \%\end{array}$ & Road, sealed or unsealed: $158,1.5 \%$ \\
\hline & & Footpath/path/foot track: $117,1.1 \%$ \\
\hline & & Classroom: $103,1 \%$ \\
\hline & & Veranda or balcony, ramp: $67,0.6 \%$ \\
\hline & & Court tennis, squash, etc.: $57,0.5 \%$ \\
\hline & & $\begin{array}{l}\text { Paddock/field/camping ground: } \\
\qquad 40,0.4 \%\end{array}$ \\
\hline & & Miscellaneous: $38,0.4 \%$ \\
\hline & & Skate park/ice rink: $37,0.35$ \\
\hline & & Hall foyer: $33,0.3 \%$ \\
\hline & & Garage, workshop, shed: $29,0.3 \%$ \\
\hline & & $\begin{array}{l}\text { Area beneath a building or structure: } \\
\qquad 22,0.2 \%\end{array}$ \\
\hline & & Beach: $21,0.2 \%$ \\
\hline & & Toilet: $20,0.2 \%$ \\
\hline
\end{tabular}

Table VII. Presentations of proportions of males and females within age groups

\begin{tabular}{|lccc|}
\hline Age group categories & Male & Female & Total \\
\hline Infants (0 years) & $402,57.8 \%$ & $293,42.2 \%$ & 695 \\
\hline $1-2$ years & $2144,61.1 \%$ & $1367,38.9 \%$ & 3511 \\
\hline $3-4$ years & $1364,62.0 \%$ & $837,38.0 \%$ & 2201 \\
\hline $5-9$ years & $1965,63.0 \%$ & $1155,37.0 \%$ & 3120 \\
\hline $10-12$ years & $827,69.1 \%$ & $369,30.9 \%$ & 1196 \\
\hline Total & $6702,62.5 \%$ & $402,37.5 \%$ & $10,723,100 \%$ \\
\hline
\end{tabular}


Table VIII. Mechanism of injury by gender, $p<0.001$

\begin{tabular}{|lcccc|}
\hline Mechanism of injury & N, $\%$ & Male & Female & Total \\
\hline Contact with static object & $2238,20.9 \%$ & $1431,63.9 \%$ & $807,36.1 \%$ & $2238,100 \%$ \\
\hline Cutting, tearing & $1359,12.7 \%$ & $874,64.3 \%$ & $485,35.7 \%$ & $1359,100 \%$ \\
\hline Other fall & $1085,10.1 \%$ & $690,63.6 \%$ & $395,36.4 \%$ & $1085,100 \%$ \\
\hline Fall by stumbling, tripping on same level & $(1056,9.8 \%)$ & $(653,61.8 \%)$ & $(403,38.2 \%)$ & $(1056,100 \%)$ \\
\hline Fall/jump from lesser height (less than 1 m) & $(980,9.1 \%)$ & $(584,59.6 \%)$ & $(137,46.9 \%)$ & $(980,100 \%)$ \\
\hline Contact with moving object & $(956,8.9 \%)$ & $(604,63.2 \%)$ & $(352,36.8 \%)$ & $(956,100 \%)$ \\
\hline Contact with person & $(895,8.3)$ & $(615,68.7 \%)$ & $(280,31.3 \%)$ & $(895,100 \%)$ \\
\hline Miscellaneous and unspecified mechanism of & $(469,4.4 \%)$ & $(297,63.3 \%)$ & $(172,36.7 \%)$ & $(469,100 \%)$ \\
injury & $(380,3.5 \%)$ & $(201,52.9 \%)$ & $(179,47.1 \%)$ & $(380,100 \%)$ \\
\hline Bite, sting by animal, human, insect & $(292,2.7 \%)$ & $(155,53.1 \%)$ & $(137,46.9 \%)$ & $(292,100 \%)$ \\
\hline Fall on or from stairs & $(231,2.2 \%)$ & $(145,62.8 \%)$ & $(86,37.2 \%)$ & $(231,100 \%)$ \\
\hline Other, unspecified contact & $(201,1.9 \%)$ & $(112,55.7 \%)$ & $(89,44.3 \%)$ & $(201,100 \%)$ \\
\hline Burns & $(193,1.8 \%)$ & $(117,60.6 \%)$ & $(76,39.4 \%)$ & $(193,100 \%)$ \\
\hline Fall/jump from greater height (more than 1 m) & $(180,1.7 \%)$ & $(84,46.7 \%)$ & $(96,53.3 \%)$ & $(180,100 \%)$ \\
\hline Contact with animal & $(162,1.5)$ & $(115,71.0 \%)$ & $(47,29.0 \%)$ & $(162,100 \%)$ \\
\hline All pinch, crush, puncture, pierce & $(46,0.4 \%)$ & $(25,54.3 \%)$ & $(21,45.7 \%)$ & $(46,100 \%)$ \\
\hline Contact, splash, spill & $(10723,100 \%)$ & $(6702,62.5 \%)$ & $(4021,37.5 \%)$ & $(10723,100 \%)$ \\
\hline Total & & & &
\end{tabular}

Table IX. Activity by gender, $p<0.001$

\begin{tabular}{|lcccc|}
\hline Activities & $\boldsymbol{N}, \boldsymbol{\%}$ & Male & Female & Total \\
\hline Playing & $3809,35.5 \%$ & $2402,63.1 \%$ & $1407,36.9 \%$ & $3809,100 \%$ \\
\hline Resting, sleeping, eating, other & $1583,14.8 \%$ & $951,60.1 \%$ & $632,39.9 \%$ & $1583,100 \%$ \\
\hline Unspecified activity & $1452,13.5 \%$ & $875,60.3 \%$ & $577,39.7 \%$ & $1452,100 \%$ \\
\hline Other specified activity & $1083,10.1 \%$ & $635,58.6 \%$ & $448,41,4 \%$ & $1083,100 \%$ \\
\hline Being nursed or care for & $988,9.2 \%$ & $577,58.4 \%$ & $411,41,6 \%$ & $988,100 \%$ \\
\hline Engaged in formal education & $507,4.7 \%$ & $329,64.9 \%$ & $178,35.1 \%$ & $507,100 \%$ \\
\hline Roller blading, skateboarding & $272,2.5 \%$ & $208,76.5 \%$ & $64,23.5 \%$ & $272,100 \%$ \\
\hline Bicycling & $262,2.4 \%$ & $193,73.7 \%$ & $69,26.3 \%$ & $262,100 \%$ \\
\hline Football, rugby, soccer & $216,2 \%$ & $204,94.4 \%$ & $12,5.6 \%$ & $216,100 \%$ \\
\hline Swimming & $162,1.5 \%$ & $101,62.3 \%$ & $61,37.7 \%$ & $162,100 \%$ \\
\hline Trampolining & $141,1.3 \%$ & $85,60.3 \%$ & $56,39.7 \%$ & $141,100 \%$ \\
\hline $\begin{array}{l}\text { Ball sport with racquet, bat } \\
\text { (baseball, cricket, hockey) }\end{array}$ & $99,0.9 \%$ & $65,65.7 \%$ & $34,34.3 \%$ & $99,100 \% 0$ \\
\hline Gymnastics and martial arts & $40,0.4 \%$ & $17,42.5 \%$ & $23,57.5 \%$ & $940,100 \%$ \\
\hline Basketball, netball & $31,0.3 \%$ & $14,45.2 \%$ & $17,54.8 \%$ & $31,100 \%$ \\
\hline $\begin{array}{l}\text { Motorised sports (go-kart, } \\
\text { minibike, motor cross) }\end{array}$ & $30,0.3 \%$ & $21,70 \%$ & $9,30 \%$ & $30,100 \%$ \\
\hline Fishing and boating & $21,0.2 \%$ & $15,71.4 \%$ & $6,28.6 \%$ & $21,100 \%$ \\
\hline Horse riding & $14,0.1 \%$ & $1,7.1 \%$ & $13,92.9 \%$ & $14,100 \%$ \\
\hline Jogging, walking & $9.0 .1 \%$ & $5,55.6 \%$ & $4,44.4 \%$ & $9,100 \%$ \\
\hline Water skiing & $4,0 \%$ & $4,100 \%$ & $0,0 \%$ & $4,100 \%$ \\
\hline Total & $10,723,100 \%$ & $6702,62.5 \%$ & $4021,37.5 \%$ & $10,723,100 \%$ \\
\hline
\end{tabular}


Table X. Nature of injury by gender, $p<0.001$

\begin{tabular}{|lcccc|}
\hline Nature of injury & $\boldsymbol{N}, \%$ & Male & Female & Total \\
\hline Open wound (excludes eye) & $4527,42.2 \%$ & $2895,63.9 \%$ & $1632,36.1$ & $4527,100 \%$ \\
\hline Superficial (incl. bruise; excl. eye) & $3303,30.8 \%$ & $1971,59.7 \%$ & $1332,40.3 \%$ & $3303,100 \%$ \\
\hline $\begin{array}{l}\text { Eye injury (excl. foreign body in external eye } \\
{[14.1] \text {; includes burn) }}\end{array}$ & $1790,16.7$ & $1164,65.0 \%$ & $626,35.0 \%$ & $1790,100 \%$ \\
\hline Miscellaneous or unspecified & $313,2.9 \%$ & $188,60.1 \%$ & $125,39.9 \%$ & $313,100 \%$ \\
\hline Fracture and dislocation (excludes tooth) & $237,2.2 \%$ & $150,63.3 \%$ & $87,36.7 \%$ & $237,100 \%$ \\
\hline Dental injury (incl. fractured tooth & $227,2.1 \%$ & $148,65.2 \%$ & $79,34.8 \%$ & $227,100 \%$ \\
\hline Burn or corrosion (excl. eye) & $176,1.6$ & $99,56.3 \%$ & $77,34 \%$ & $176,100 \%$ \\
\hline Intracranial injury (includes concussion) & $94,0.9 \%$ & $59,62.8 \%$ & $35,37.2 \%$ & $94,100 \%$ \\
\hline Effect of venom; any insect bite & $56,0.5 \%$ & $28,50 \%$ & $28,50 \%$ & $65,100 \%$ \\
\hline Total & $10,723,100 \%$ & $6702,62.5 \%$ & $4021,37.5 \%$ & $10,723,100 \%$ \\
\hline
\end{tabular}

Table XI. Type of place by gender, $p<0.001$

\begin{tabular}{|lcccc|}
\hline Type of place & N, $\%$ & Male & Female & Total \\
\hline Home (includes farmhouse) & $9686,65.1 \%$ & $4257,60.9 \%$ & $2729,39.1 \%$ & $6986,100 \%$ \\
\hline Unspecified place & $834,7.8 \%$ & $499,59.8 \%$ & $335,40.2 \%$ & $834,100 \%$ \\
\hline Primary, secondary school & $741,6.9 \%$ & $485,65.5 \%$ & $256,34.5 \%$ & $741,100 \%$ \\
\hline Childcare, preschool centre & $428,4.0 \%$ & $292,68.2 \%$ & $136,31.8 \%$ & $428,100 \%$ \\
\hline Public park & $372,3.5 \%$ & $249,66.9 \%$ & $123,33.1 \%$ & $372,100 \%$ \\
\hline Miscellaneous specified place & $229,2.1 \%$ & $148,64.6 \%$ & $81,35.4 \%$ & $229,100 \%$ \\
\hline $\begin{array}{l}\text { Recreation area (informal), specified, incl. } \\
\text { amusement park, aquatic recreation centre }\end{array}$ & $299,2.1 \%$ & $151,65.9 \%$ & $78,34.1 \%$ & $299,100 \%$ \\
\hline Oval, fields, pitch & $277,2.6 \%$ & $238,85.9 \%$ & $39,14.1 \%$ & $277,100 \%$ \\
\hline Street or highway (Public road) & $236,2.2 \%$ & $154,65.3 \%$ & $82,34.7 \%$ & $236,100 \%$ \\
\hline $\begin{array}{l}\text { Trade or service area, incl. shopping centre, } \\
\text { restaurant }\end{array}$ & $200,1.9 \%$ & $117,58.5 \%$ & $83,41.5 \%$ & $200,100 \%$ \\
\hline Other and unspecified sports area & $78,0.7 \%$ & $44,56.4 \%$ & $34,43.6 \%$ & $78,100 \%$ \\
\hline Farm (excl. farmhouse) & $44,0.4 \%$ & $22,50.0 \%$ & $22,50.0 \%$ & $44,100 \%$ \\
\hline Bush, remote or undeveloped place & $42,0.4 \%$ & $28,66.7 \%$ & $14,33.3 \%$ & $42,100 \%$ \\
\hline Hospital, medical clinic, or other health service & $27,0.3 \%$ & $18,66.7 \%$ & $9,33.3 \%$ & $27,100 \%$ \\
\hline Total & $10,723,100 \%$ & $6702,100 \%$ & $4021,100 \%$ & $10,723,100 \%$ \\
\hline
\end{tabular}

ly to be admitted (20.0\%). Infants were more likely to have head injuries compared to face injuries (55.7\%), while 10-12-year-olds were more likely to have injuries coded as 'body location not required' (31.9\%), which are injuries specific to a part of the body such as foreign body in eye, or concussion.

Mechanisms of injuries by age groups

There was an association between mechanism of injury and age group $\left(\chi^{2}=1873.67, \mathrm{~d} f=60\right.$, $p<0.001$ ). Fall/jump from lesser height (less than $1 \mathrm{~m}$ ) was the most common mechanism of injury among infants, whereas contact with static object was the most frequent cause of injuries in 1-2, 3-4, and 5-9 years age groups. Contact with another person was the most common mechanism of injury in the 10-12 years age group. Cutting and tearing was the second most common mechanism of injury in both the 1-2 years and 3-4 years age groups, while contact with a moving object was the second most common mechanism of injury in both the 5-9 years and $10-12$ years age groups. Contact with a static object was the second mechanism of injury in infants (Table XIII).

Type of place where the injuries occurred

The association between type of place where the children were injured and age group was significant $\left(\chi^{2}=2713.20, \mathrm{~d} f=52, p<0.001\right)$. The primary area where injury occurred was found to be in the home in all age groups, followed by unspecified place for both infants and the 1-2 years age group, whereas 'childcare preschool' centre was the second main type of place for the 3-4 years age group. 'Primary, secondary school' was 
Table XII. Intent, triage, mode of separation, and body region by age groups

\begin{tabular}{|c|c|c|c|c|c|c|c|}
\hline Variables & & $\begin{array}{l}\text { Infants } \\
\text { (0 years) }\end{array}$ & $\begin{array}{c}1-2 \\
\text { years }\end{array}$ & $\begin{array}{c}3-4 \\
\text { years }\end{array}$ & $\begin{array}{c}5-9 \\
\text { years }\end{array}$ & $\begin{array}{l}10-12 \\
\text { years }\end{array}$ & Total \\
\hline \multirow[t]{3}{*}{$\begin{array}{l}\text { Intent } \\
p<0.001\end{array}$} & $\begin{array}{l}\text { Accident; injury was } \\
\text { not intended }\end{array}$ & $\begin{array}{c}679 \\
97.7 \%\end{array}$ & $\begin{array}{l}3481 \\
99.1 \%\end{array}$ & $2179,99 \%$ & $3059,98 \%$ & $\begin{array}{l}1144, \\
95.7 \%\end{array}$ & $\begin{array}{l}10542 \\
98.3 \%\end{array}$ \\
\hline & $\begin{array}{c}\text { Event of unclassified or } \\
\text { undetermined intent }\end{array}$ & $11,1.6 \%$ & $22,0.6 \%$ & $17,0.8 \%$ & $37,1.2 \%$ & $14,1.2 \%$ & $101,0.9 \%$ \\
\hline & $\begin{array}{c}\text { Assault/harm by other } \\
\text { person }\end{array}$ & $5,0.7 \%$ & $8,0.2 \%$ & $5,0.2 \%$ & $24,0.8 \%$ & $38,3.2 \%$ & $80,0.7 \%$ \\
\hline \multirow{4}{*}{$\begin{array}{l}\text { Severity } \\
\text { (Triage) } \\
p<0.001\end{array}$} & Emergency & $43,6.2 \%$ & $151,4.3 \%$ & $87,4 \%$ & $191,6.1 \%$ & $\begin{array}{c}121 \\
10.1 \%\end{array}$ & $593,5.5 \%$ \\
\hline & Urgent (30 min) & $\begin{array}{c}190 \\
27.3 \%\end{array}$ & $\begin{array}{c}611 \\
17.4 \%\end{array}$ & $\begin{array}{l}387 \\
17.6 \%\end{array}$ & $\begin{array}{c}733 \\
23.5 \%\end{array}$ & $\begin{array}{l}363 \\
30.4 \%\end{array}$ & $\begin{array}{l}2284 \\
21.3 \%\end{array}$ \\
\hline & Semi urgent (60 min) & $438,63 \%$ & $\begin{array}{l}2581 \\
73.5 \%\end{array}$ & $\begin{array}{l}1604 \\
72.9 \%\end{array}$ & $\begin{array}{l}2035 \\
65.2 \%\end{array}$ & $\begin{array}{l}657 \\
54.9 \%\end{array}$ & $\begin{array}{l}7315 \\
68.2 \%\end{array}$ \\
\hline & $\begin{array}{l}\text { Non urgent } \\
(120 \mathrm{~min})\end{array}$ & $24,3.5 \%$ & $168,4.8 \%$ & $123,5.6 \%$ & $160,5.1 \%$ & $55,4.6 \%$ & $530,4.9 \%$ \\
\hline \multirow{3}{*}{$\begin{array}{l}\text { Outcome } \\
\text { or ED visit } \\
\text { (mode- of } \\
\text { separation) } \\
p<0.001\end{array}$} & $\begin{array}{c}\text { ED service event } \\
\text { completed-discharged }\end{array}$ & $\begin{array}{c}543 \\
78.1 \%\end{array}$ & $\begin{array}{l}2863 \\
81.5 \%\end{array}$ & $\begin{array}{l}1824 \\
82.9 \%\end{array}$ & $\begin{array}{l}2650 \\
84.9 \%\end{array}$ & $\begin{array}{l}1024 \\
85.6 \%\end{array}$ & $\begin{array}{l}1661 \\
15.5 \%\end{array}$ \\
\hline & $\begin{array}{c}\text { Admitted to same or } \\
\text { other hospital }\end{array}$ & $\begin{array}{c}139 \\
20.0 \%\end{array}$ & $\begin{array}{c}582, \\
16.6 \%\end{array}$ & $352,16 \%$ & $\begin{array}{c}435 \\
13.9 \% \\
\end{array}$ & $\begin{array}{c}153 \\
12.8 \%\end{array}$ & $\begin{array}{l}8904, \\
83.0 \%\end{array}$ \\
\hline & $\begin{array}{l}\text { Did not wait/left after } \\
\text { treatment commenced }\end{array}$ & $13,1.9 \%$ & $66,1.9 \%$ & $25,1.1 \%$ & $35,1.1 \%$ & $19,1.6 \%$ & $158,1.5 \%$ \\
\hline \multirow{4}{*}{$\begin{array}{l}\text { Body } \\
\text { region } \\
p<0.001\end{array}$} & Head (excludes face) & $\begin{array}{c}387 \\
55.7 \%\end{array}$ & $\begin{array}{l}1499 \\
42.7 \%\end{array}$ & $\begin{array}{c}863 \\
39.2 \%\end{array}$ & $\begin{array}{c}987, \\
31.6 \%\end{array}$ & $\begin{array}{c}346 \\
28.9 \%\end{array}$ & $\begin{array}{l}4082 \\
38.1 \%\end{array}$ \\
\hline & Face (excludes eye) & $\begin{array}{l}232 \\
33.4 \%\end{array}$ & $\begin{array}{l}1555, \\
44.3 \%\end{array}$ & $\begin{array}{l}977 \\
44.4 \%\end{array}$ & $\begin{array}{l}1193 \\
38.2 \%\end{array}$ & $\begin{array}{l}434 \\
36.3 \%\end{array}$ & $\begin{array}{l}4391 \\
40.9 \%\end{array}$ \\
\hline & $\begin{array}{l}\text { Multiple injuries } \\
\text { (involving more than } \\
\text { one bodily location }\end{array}$ & $11,1.6 \%$ & $45,1.3 \%$ & $13,0.6 \%$ & $63,2.1 \%$ & $35,2.9 \%$ & $168,1.6 \%$ \\
\hline & $\begin{array}{l}\text { Body location not } \\
\text { required }\end{array}$ & $65,9.4 \%$ & $\begin{array}{l}412, \\
11.7 \%\end{array}$ & $\begin{array}{l}348, \\
15.8 \%\end{array}$ & $\begin{array}{l}876 \\
28.1 \%\end{array}$ & $\begin{array}{c}381 \\
31.9 \%\end{array}$ & $\begin{array}{l}2012 \\
19.4 \%\end{array}$ \\
\hline
\end{tabular}

the second most frequent type of place for both 5-9 years and $10-12$ years age groups (Table XIV).

Part of place where injuries occurred

There was an association between part of place and age group $\left(\chi^{2}=2490.74, \mathrm{~d} f=116, p<0.001\right.$, but $\chi^{2}$ assumptions were not fulfilled, and it was not possible to perform an exact test). Children sustained most head and facial injuries in 'unspecified part of place' for all age groups, followed by the bedroom for both infants and 3-4 years age groups, while 'living, family, rumpus room' and 'garden, park, national park/backyard' were the second most common part of place where injury occurred for both 1-2 years and 5-9 year age groups, respectively, as shown in Table XV. Oval/field was the second most common part of place where children sustained injury for 10-12 years age group.

Activities when injuries occurred

The association between activity during which children were injured and age group was significant $\left(\chi^{2}=4165.61, \mathrm{~d} f=72, p<0.001\right.$, but $\chi^{2}$ assumptions were not fulfilled, and it was not possible to perform an exact test). The majority of children were injured during playing in the 1-2 years, 3-4 years, 5-9 years, and 10-12 years age groups, and most infants were injured when being nursed or cared for. 'Resting, sleeping, eating, other' was the second most common activity when injury occurred for infants in the 1-2 years and 3-4 years age groups, while unspecified activity was the second most common activity for children aged 5-9 and 10-12 years (Table XVI).

Nature of injuries by age group

The association between nature of injury and age group was significant $\left(\chi^{2}=1173.10, \mathrm{~d} f=32\right.$, $p<0.001)$. For infants and 10-12-year-olds, superficial injuries were the most common $(59.6 \%$, 29.85\%), while for 1-2- and 3-4-year-olds open wounds were the most common nature of injury $(49.0 \%, 51.7 \%, 38.85 \%) .10-12$-year-olds had proportionally the most eye injuries and fractures ( $27.4 \%, 6.1 \%$ of total injuries among $10-12$-yearolds) (Table XVII). 
Table XIII. Mechanism of injury by age group, $p<0.001$

\begin{tabular}{|c|c|c|c|c|c|c|}
\hline Ranking & $<1$ year & $1-2$ years & $3-4$ years & 5-9 years & $10-2$ years & Total \\
\hline 1 & $\begin{array}{l}\text { Fall/jump from } \\
\text { lesser height } \\
\text { (less than } 1 \mathrm{~m} \text { ) } \\
(160,23 \%)\end{array}$ & $\begin{array}{l}\text { Contact with } \\
\text { static object } \\
(857,24.4 \%)\end{array}$ & $\begin{array}{l}\text { Contact with } \\
\text { static object } \\
(542,24.6 \%)\end{array}$ & $\begin{array}{l}\text { Contact with } \\
\text { static object } \\
(585,18.8 \%)\end{array}$ & $\begin{array}{c}\text { Contact with } \\
\text { person } \\
(275,23 \%)\end{array}$ & 2419 \\
\hline 2 & $\begin{array}{l}\text { Contact with } \\
\text { static object } \\
(125,18 \%)\end{array}$ & $\begin{array}{l}\text { Cutting, tearing } \\
(539,15.4 \%)\end{array}$ & $\begin{array}{l}\text { Cutting, tearing } \\
(337,15.3 \%)\end{array}$ & $\begin{array}{l}\text { Contact with } \\
\text { moving object } \\
(399,12.8 \%)\end{array}$ & $\begin{array}{l}\text { Contact with } \\
\text { moving object } \\
(235,19.6 \%)\end{array}$ & 1635 \\
\hline 3 & $\begin{array}{c}\text { Other falls } \\
(115,16.5 \%)\end{array}$ & $\begin{array}{c}\text { Fall by stumbling, } \\
\text { tripping on same } \\
\text { level } \\
(521,14.8 \%)\end{array}$ & $\begin{array}{c}\text { Fall by stumbling, } \\
\text { tripping on same } \\
\text { level } \\
(225,10.2 \%)\end{array}$ & $\begin{array}{l}\text { Contact with } \\
\text { person } \\
(388,12.4 \%)\end{array}$ & $\begin{array}{l}\text { Contact with } \\
\text { static object } \\
(129,10.8 \%)\end{array}$ & 1378 \\
\hline 4 & $\begin{array}{c}\text { Fall by stumbling, } \\
\text { tripping on same } \\
\text { level } \\
(64,9.2 \%)\end{array}$ & $\begin{array}{l}\text { Fall/jump from } \\
\text { lesser height } \\
\text { (less than } 1 \mathrm{~m}) \\
(402,11.4 \%)\end{array}$ & $\begin{array}{c}\text { Other fall } \\
(222,10.1 \%)\end{array}$ & $\begin{array}{c}\text { Cutting, tearing } \\
(343,11 \%)\end{array}$ & $\begin{array}{c}\text { Other fall } \\
(105,8.8 \%)\end{array}$ & 1136 \\
\hline 5 & $\begin{array}{l}\text { Cutting, tearing } \\
\quad(51,7.3 \%)\end{array}$ & $\begin{array}{l}\text { Other falls } \\
(337,9.6 \%)\end{array}$ & $\begin{array}{l}\text { Fall/jump from } \\
\text { lesser height } \\
\text { (less than } 1 \mathrm{~m}) \\
(213,9.7 \%)\end{array}$ & $\begin{array}{c}\text { Other fall } \\
(306,9.8 \%)\end{array}$ & $\begin{array}{l}\text { Cutting, tearing } \\
(89,7.4 \%)\end{array}$ & 996 \\
\hline 6 & $\begin{array}{c}\text { Contact with } \\
\text { moving object } \\
(31,4.5 \%)\end{array}$ & $\begin{array}{c}\text { Fall on or from } \\
\text { stairs } \\
(138,3.9 \%)\end{array}$ & $\begin{array}{c}\text { Contact with } \\
\text { moving object } \\
(153,7 \%)\end{array}$ & $\begin{array}{l}\text { Miscellaneous } \\
\text { and un-specified } \\
\text { mechanism of } \\
\text { injury } \\
(220,7.1 \%)\end{array}$ & $\begin{array}{l}\text { Miscellaneous } \\
\text { and un-specified } \\
\text { mechanism of } \\
\text { injury } \\
(85,7.1 \%)\end{array}$ & 627 \\
\hline 7 & $\begin{array}{c}\text { Contact with } \\
\text { person } \\
(30,4.3 \%)\end{array}$ & $\begin{array}{l}\text { Contact with } \\
\text { moving object } \\
(138,3.9 \%)\end{array}$ & $\begin{array}{c}\text { Contact with } \\
\text { person } \\
(112,5.1 \%)\end{array}$ & $\begin{array}{c}\text { Fall by stumbling, } \\
\text { tripping on same } \\
\text { level } \\
(190,6.1 \%)\end{array}$ & $\begin{array}{c}\text { Fall by stumbling, } \\
\text { tripping on same } \\
\text { level } \\
(56,4.7 \%)\end{array}$ & 526 \\
\hline 8 & $\begin{array}{c}\text { Fall on or from } \\
\text { stairs } \\
(29,4.2 \%)\end{array}$ & $\begin{array}{c}\text { Burns } \\
(95,2.7 \%)\end{array}$ & $\begin{array}{l}\text { Bite, sting by } \\
\text { animal, human, } \\
\text { insect } \\
(91,4.1 \%)\end{array}$ & $\begin{array}{l}\text { Fall/jump from } \\
\text { lesser height } \\
\text { (less than } 1 \mathrm{~m} \text { ) } \\
(169,5.4 \%)\end{array}$ & $\begin{array}{l}\text { Bite, sting by } \\
\text { animal, human, } \\
\text { insect } \\
(50,4.2 \%)\end{array}$ & 434 \\
\hline 9 & $\begin{array}{c}\text { Burns } \\
(28,4 \%)\end{array}$ & $\begin{array}{l}\text { Contact with } \\
\text { person } \\
(90,2.6 \%)\end{array}$ & $\begin{array}{l}\text { Miscellaneous } \\
\text { and un-specified } \\
\text { mechanism of } \\
\text { injury } \\
(61,2.8 \%)\end{array}$ & $\begin{array}{l}\text { Bite, sting by } \\
\text { animal, human, } \\
\text { insect } \\
(140,4.5 \%)\end{array}$ & $\begin{array}{c}\text { Other, } \\
\text { unspecified } \\
\text { contact } \\
(37,3.1 \%)\end{array}$ & 356 \\
\hline 10 & $\begin{array}{c}\text { Fall/jump from } \\
\text { greater height } \\
\text { (more than } 1 \mathrm{~m}) \\
(18,2.6 \%)\end{array}$ & $\begin{array}{l}\text { Bite, sting, by } \\
\text { animal, human, } \\
\text { insect } \\
(90,2.6 \%)\end{array}$ & $\begin{array}{c}\text { Fall on or from } \\
\text { stairs } \\
(54,2.4 \%)\end{array}$ & $\begin{array}{c}\text { Other, } \\
\text { unspecified } \\
\text { contact } \\
(75,2.4 \%) \\
\end{array}$ & $\begin{array}{l}\text { Fall/jump from } \\
\text { lesser height } \\
\text { (less than } 1 \mathrm{~m} \text { ) } \\
(36,3 \%)\end{array}$ & 273 \\
\hline 11 & $\begin{array}{l}\text { Miscellaneous } \\
\text { and un-specified } \\
\text { mechanism of } \\
\text { injury } \\
(17,2.4 \%)\end{array}$ & $\begin{array}{l}\text { Miscellaneous } \\
\text { and un-specified } \\
\text { mechanism of } \\
\text { injury } \\
(86,2.4 \%)\end{array}$ & $\begin{array}{c}\text { Other, } \\
\text { unspecified } \\
\text { contact } \\
(47,2.1 \%)\end{array}$ & $\begin{array}{c}\text { Fall on or from } \\
\text { stairs } \\
(65,2.1 \%)\end{array}$ & $\begin{array}{l}\text { All Pinch, crush, } \\
\text { puncture, pierce } \\
(26,2.2 \%)\end{array}$ & 241 \\
\hline 12 & $\begin{array}{c}\text { Other, } \\
\text { unspecified } \\
\text { contact } \\
(11,1.6 \%) \\
\end{array}$ & $\begin{array}{c}\text { Fall/jump from } \\
\text { greater height } \\
\text { (more than } 1 \mathrm{~m}) \\
(77,2.2 \%)\end{array}$ & $\begin{array}{l}\text { Contact with } \\
\text { animal } \\
(46,2.1 \%)\end{array}$ & $\begin{array}{l}\text { Contact with } \\
\text { animal } \\
(64,2.1 \%)\end{array}$ & $\begin{array}{c}\text { Contact with } \\
\text { animal } \\
(23,1.9 \%)\end{array}$ & 221 \\
\hline 13 & $\begin{array}{l}\text { Bite, sting, by } \\
\text { animal, human, } \\
\text { insect } \\
(9,1.3 \%)\end{array}$ & $\begin{array}{c}\text { Other, } \\
\text { unspecified } \\
\text { contact } \\
(61,1.7 \%)\end{array}$ & $\begin{array}{l}\text { All pinch, crush, } \\
\text { puncture, pierce } \\
\quad(40,1.8 \%)\end{array}$ & $\begin{array}{l}\text { All pinch, crush, } \\
\text { puncture, pierce } \\
(62,2 \%)\end{array}$ & $\begin{array}{c}\text { Burns } \\
(22,1.8 \%)\end{array}$ & 194 \\
\hline 14 & $\begin{array}{c}\text { Contact with } \\
\text { animal } \\
(3,0.4 \%)\end{array}$ & $\begin{array}{c}\text { Contact with } \\
\text { animal } \\
(44,1.3 \%)\end{array}$ & $\begin{array}{c}\text { Fall/jump from } \\
\text { greater height } \\
\text { (more than } 1 \mathrm{~m}) \\
(38,1.7 \%)\end{array}$ & $\begin{array}{c}\text { Fall/jump from } \\
\text { greater height } \\
\text { (more than } 1 \mathrm{~m} \text { ) } \\
(48,1.5 \%)\end{array}$ & $\begin{array}{l}\text { Fall/jump from } \\
\text { greater height } \\
\text { (more than } 1 \mathrm{~m}) \\
(12,1 \%)\end{array}$ & 145 \\
\hline
\end{tabular}


Table XIII. Cd.

\begin{tabular}{|lcccccc|}
\hline Ranking & $<\mathbf{1}$ year & $\mathbf{1 - 2}$ years & $\mathbf{3 - 4}$ years & $\mathbf{5 - 9}$ years & $\mathbf{1 0 - 2}$ years & Total \\
\hline 15 & $\begin{array}{c}\text { All Pinch, crush, } \\
\text { puncture, pierce } \\
(2,0.3 \%)\end{array}$ & $\begin{array}{c}\text { All Pinch, crush, } \\
\text { puncture, pierce } \\
(32,0.9 \%)\end{array}$ & $\begin{array}{c}\text { Burns } \\
(17,0.8 \%)\end{array}$ & $\begin{array}{c}\text { Burns } \\
(39,1.3 \%)\end{array}$ & $\begin{array}{c}\text { Contact, splash, } \\
\text { spill } \\
(10,0.8 \%)\end{array}$ & 100 \\
\hline 16 & $\begin{array}{c}\text { Contact, splash, } \\
\text { spill } \\
(2,0.3 \%)\end{array}$ & $\begin{array}{c}\text { Contact, splash, } \\
\text { spill } \\
(4,0.1 \%)\end{array}$ & $\begin{array}{c}\text { Contact, splash, } \\
\text { spill } \\
(3,0.1 \%)\end{array}$ & $\begin{array}{c}\text { Contact, splash, } \\
\text { spill } \\
(27,0.9 \%)\end{array}$ & $\begin{array}{c}\text { Fall on or from } \\
\text { stairs } \\
(6,0.5 \%)\end{array}$ & 42 \\
\hline Total & 695 & 3511 & 2201 & 3120 & 1196 & 10,723 \\
\hline
\end{tabular}

Table XIV. Type of place where the injuries occurred, $p<0.001$

\begin{tabular}{|c|c|c|c|c|c|c|}
\hline Ranking & $\begin{array}{c}<1 \text { year age } \\
\text { group } \\
n=695\end{array}$ & $\begin{array}{c}1-2 \text { years age } \\
\text { group } \\
n=3511\end{array}$ & $\begin{array}{l}\text { 3-4 years } \\
\text { age group } \\
n=2201\end{array}$ & $\begin{array}{c}\text { 5-9 years age } \\
\text { group } \\
n=3120\end{array}$ & $\begin{array}{c}10-12 \text { years age } \\
\text { group } \\
n=1196\end{array}$ & $\begin{array}{c}\text { Total } \\
10723\end{array}$ \\
\hline 1 & $\begin{array}{l}\text { Home (includes } \\
\text { farmhouse) } \\
(596,85.8 \%)\end{array}$ & $\begin{array}{l}\text { Home (includes } \\
\text { farmhouse) } \\
(2714,77.3 \%)\end{array}$ & $\begin{array}{l}\text { Home (includes } \\
\text { farmhouse) } \\
(1524,69.2 \%)\end{array}$ & $\begin{array}{l}\text { Home (includes } \\
\text { farmhouse) } \\
(1709,54.8 \%)\end{array}$ & $\begin{array}{l}\text { Home (includes } \\
\text { farmhouse) } \\
(443,37 \%)\end{array}$ & 6986 \\
\hline 2 & $\begin{array}{l}\text { Unspecified } \\
\text { place } \\
(45,6.5 \%)\end{array}$ & $\begin{array}{l}\text { Unspecified } \\
\text { place } \\
(237,6.8 \%)\end{array}$ & $\begin{array}{c}\text { Childcare } \\
\text { preschool centre } \\
(187,8.5 \%)\end{array}$ & $\begin{array}{c}\text { Primary, } \\
\text { secondary school } \\
(480,15.5 \%)\end{array}$ & $\begin{array}{c}\text { Primary, } \\
\text { secondary school } \\
(240,20.1 \%)\end{array}$ & 1189 \\
\hline 3 & $\begin{array}{c}\text { Trade or service } \\
\text { area, incl. } \\
\text { shopping centre, } \\
\text { restaurant } \\
(18,2.6 \%)\end{array}$ & $\begin{array}{l}\text { Childcare, } \\
\text { preschool centre } \\
(202,5.8 \%)\end{array}$ & $\begin{array}{l}\text { Unspecified } \\
\text { place } \\
(168,7.6 \%)\end{array}$ & $\begin{array}{l}\text { Unspecified } \\
\text { place } \\
(263,8.4 \%)\end{array}$ & $\begin{array}{l}\text { Oval, fields, pitch } \\
(165,13.8 \%)\end{array}$ & 816 \\
\hline 4 & $\begin{array}{c}\text { Public park } \\
(8,1.2 \%)\end{array}$ & $\begin{array}{c}\text { Public park } \\
(94,2.7 \%)\end{array}$ & $\begin{array}{l}\text { Public park } \\
(97,4.4 \%)\end{array}$ & $\begin{array}{l}\text { Public park } \\
(125,4 \%)\end{array}$ & $\begin{array}{c}\text { Unspecified place } \\
(121,10.1 \%)\end{array}$ & 445 \\
\hline 5 & $\begin{array}{c}\text { Childcare, } \\
\text { preschool centre } \\
(6,0.9 \%)\end{array}$ & $\begin{array}{l}\text { Trade or service } \\
\text { area, incl. } \\
\text { shopping centre, } \\
\text { restaurant } \\
(85,2.4 \%)\end{array}$ & $\begin{array}{l}\text { Trade or service } \\
\text { area, incl. } \\
\text { shopping centre, } \\
\text { restaurant } \\
(52,2.4 \%)\end{array}$ & $\begin{array}{c}\text { Street or } \\
\text { highway (Public } \\
\text { road) } \\
(108,3.5 \%)\end{array}$ & $\begin{array}{l}\text { Street or highway } \\
\text { (Public road) } \\
(52,4.3 \%)\end{array}$ & 303 \\
\hline 6 & $\begin{array}{c}\text { Street or } \\
\text { highway (Public } \\
\text { road) } \\
(6,0.9 \%)\end{array}$ & $\begin{array}{c}\text { Miscellaneous } \\
\text { specified place } \\
(67,1.9 \%)\end{array}$ & $\begin{array}{l}\text { Recreation } \\
\text { area (informal), } \\
\text { specified, incl. } \\
\text { amusement } \\
\text { park, aquatic } \\
\text { recreation centre } \\
(43,2 \%)\end{array}$ & $\begin{array}{l}\text { Oval, fields, pitch } \\
(99,3.2 \%)\end{array}$ & $\begin{array}{l}\text { Public park } \\
(48,4 \%)\end{array}$ & 263 \\
\hline 7 & $\begin{array}{l}\text { Miscellaneous } \\
\text { specified place } \\
\quad(6,0.9 \%)\end{array}$ & $\begin{array}{l}\text { Recreation } \\
\text { area (informal), } \\
\text { specified, incl. } \\
\text { amusement } \\
\text { park, aquatic } \\
\text { recreation centre } \\
(43,1.2 \%)\end{array}$ & $\begin{array}{c}\text { Street or } \\
\text { highway (Public } \\
\text { road) } \\
(43,2 \%)\end{array}$ & $\begin{array}{l}\text { Recreation } \\
\text { area (informal), } \\
\text { specified, incl. } \\
\text { amusement } \\
\text { park, aquatic } \\
\text { recreation centre } \\
(98,3.1 \%)\end{array}$ & $\begin{array}{c}\text { Recreation } \\
\text { area (informal), } \\
\text { specified, incl. } \\
\text { amusement } \\
\text { park, aquatic } \\
\text { recreation centre } \\
(43,3.6 \%)\end{array}$ & 233 \\
\hline 8 & $\begin{array}{c}\text { Bush, remote } \\
\text { or undeveloped } \\
\text { place } \\
(4,0.6 \%)\end{array}$ & $\begin{array}{c}\text { Street or } \\
\text { highway (Public } \\
\text { road) } \\
(27,0.85)\end{array}$ & $\begin{array}{c}\text { Miscellaneous } \\
\text { specified place } \\
(42,1.9 \%)\end{array}$ & $\begin{array}{c}\text { Miscellaneous } \\
\text { specified place } \\
(84,2.7 \%)\end{array}$ & $\begin{array}{c}\text { Other and } \\
\text { unspecified } \\
\text { sports area } \\
(32,2.7 \%)\end{array}$ & 189 \\
\hline 9 & $\begin{array}{l}\text { Recreation } \\
\text { area (informal), } \\
\text { specified, incl. } \\
\text { amusement } \\
\text { park, aquatic } \\
\text { recreation centre } \\
(2,0.3 \%)\end{array}$ & $\begin{array}{l}\text { Hospital, medical } \\
\text { clinic, or other } \\
\text { health service } \\
(15,0.4 \%)\end{array}$ & $\begin{array}{c}\text { Primary, } \\
\text { secondary school } \\
(16,0.7 \%)\end{array}$ & $\begin{array}{l}\text { Trade or service } \\
\text { area, incl. } \\
\text { shopping centre, } \\
\text { restaurant } \\
(40,1.3 \%)\end{array}$ & $\begin{array}{c}\text { Miscellaneous } \\
\text { specified place } \\
(30,2.5 \%)\end{array}$ & 103 \\
\hline 10 & $\begin{array}{c}\text { Primary, } \\
\text { secondary school } \\
(1,0.1 \%)\end{array}$ & $\begin{array}{c}\text { Farm (excl. } \\
\text { farmhouse) } \\
(12,0.3 \%)\end{array}$ & $\begin{array}{l}\text { Oval, fields, pitch } \\
\quad(10,0.5 \%)\end{array}$ & $\begin{array}{c}\text { Other and } \\
\text { unspecified } \\
\text { sports area } \\
(36,1.2 \%)\end{array}$ & $\begin{array}{c}\text { Bush, remote } \\
\text { or undeveloped } \\
\text { place } \\
(8,0.7 \%)\end{array}$ & 67 \\
\hline
\end{tabular}


Table XIV. $\mathrm{Cd}$.

\begin{tabular}{|c|c|c|c|c|c|c|}
\hline Ranking & $\begin{array}{c}<1 \text { year age } \\
\text { group } \\
n=695\end{array}$ & $\begin{array}{c}1-2 \text { years age } \\
\text { group } \\
n=3511\end{array}$ & $\begin{array}{c}3-4 \text { years } \\
\text { age group } \\
n=2201\end{array}$ & $\begin{array}{c}5-9 \text { years age } \\
\text { group } \\
n=3120\end{array}$ & $\begin{array}{c}10-12 \text { years age } \\
\text { group } \\
n=1196\end{array}$ & $\begin{array}{c}\text { Total } \\
10723\end{array}$ \\
\hline 11 & $\begin{array}{l}\text { Hospital, Medical } \\
\text { clinic or other } \\
\text { health service } \\
(1,0.1 \%)\end{array}$ & $\begin{array}{c}\text { Bush, remote } \\
\text { or undeveloped } \\
\text { place } \\
(5,0.1 \%)\end{array}$ & $\begin{array}{c}\text { Bush, remote } \\
\text { or undeveloped } \\
\text { place } \\
(6,0.3 \%)\end{array}$ & $\begin{array}{l}\text { Childcare, } \\
\text { preschool } \\
\text { centre } \\
(31,1 \%)\end{array}$ & $\begin{array}{c}\text { Farm (excl. } \\
\text { farmhouse) } \\
(6,0.5 \%)\end{array}$ & 49 \\
\hline 12 & $\begin{array}{c}\text { Oval, fields, pitch } \\
\quad(1,0.1 \%)\end{array}$ & $\begin{array}{c}\text { Primary, } \\
\text { secondary school } \\
(4,0.1 \%)\end{array}$ & $\begin{array}{l}\text { Hospital, Medical } \\
\text { clinic or other } \\
\text { health service } \\
(6,0.3 \%)\end{array}$ & $\begin{array}{l}\text { Farm (excl. } \\
\text { farmhouse) } \\
(24,0.8 \%)\end{array}$ & $\begin{array}{l}\text { Trade or service } \\
\text { area, incl. } \\
\text { shopping centre, } \\
\text { restaurant } \\
(5,0.4 \%)\end{array}$ & 40 \\
\hline 13 & $\begin{array}{c}\text { Other and } \\
\text { unspecified } \\
\text { sports area } \\
(1,0.1 \%)\end{array}$ & $\begin{array}{c}\text { Other and } \\
\text { unspecified } \\
\text { sports area } \\
(4,0.1 \%)\end{array}$ & $\begin{array}{l}\text { Other and } \\
\text { unspecified } \\
\text { sports area } \\
(5,0.2 \%)\end{array}$ & $\begin{array}{c}\text { Bush, remote } \\
\text { or undeveloped } \\
\text { place } \\
(19,0.6 \%)\end{array}$ & $\begin{array}{l}\text { Childcare, } \\
\text { preschool centre } \\
(2,0.2 \%)\end{array}$ & 31 \\
\hline 14 & $\begin{array}{c}\text { Farm (excl. } \\
\text { farmhouse) } \\
(0,0 \%)\end{array}$ & $\begin{array}{l}\text { Oval, fields, pitch } \\
\quad(2,0.1 \%)\end{array}$ & $\begin{array}{c}\text { Farm (excl. } \\
\text { farmhouse) } \\
(2,0.1 \%)\end{array}$ & $\begin{array}{l}\text { Hospital, Medical } \\
\text { clinic or other } \\
\text { health service } \\
(4,0.1 \%)\end{array}$ & $\begin{array}{l}\text { Hospital, medical } \\
\text { clinic, or other } \\
\text { health service } \\
(1,0.1 \%)\end{array}$ & 9 \\
\hline
\end{tabular}

Table XV. Part of place where the injuries occurred, $p<0.001$

\begin{tabular}{|c|c|c|c|c|c|c|}
\hline Ranking & $\begin{array}{c}\text { Infants (0 years) } \\
n=695\end{array}$ & $\begin{array}{c}1-2 \text { years age } \\
\text { group } \\
n=3511\end{array}$ & $\begin{array}{c}3-4 \text { years } \\
\text { age group } \\
n=2201\end{array}$ & $\begin{array}{c}\text { 5-9 years age } \\
\text { group } \\
n=3120\end{array}$ & $\begin{array}{c}10-12 \text { years age } \\
\text { group } \\
n=1196\end{array}$ & $\begin{array}{c}\text { Total } \\
10723\end{array}$ \\
\hline 1 & $\begin{array}{c}\text { Unspecified part } \\
\text { of place } \\
(247,35.5 \%)\end{array}$ & $\begin{array}{c}\text { Unspecified part } \\
\text { of place } \\
(1357,38.6 \%)\end{array}$ & $\begin{array}{c}\text { Unspecified part } \\
\text { of place } \\
(884,40.2 \%)\end{array}$ & $\begin{array}{c}\text { Unspecified part } \\
\text { of place } \\
(1221,39.1 \%)\end{array}$ & $\begin{array}{c}\text { Unspecified part } \\
\text { of place } \\
(390,32.6 \%)\end{array}$ & 4099 \\
\hline 2 & $\begin{array}{c}\text { Bedroom } \\
(133,19.1 \%)\end{array}$ & $\begin{array}{l}\text { Living, family, } \\
\text { rumpus room } \\
(365,10.4 \%)\end{array}$ & $\begin{array}{l}\text { Bedroom } \\
(175,8 \%)\end{array}$ & $\begin{array}{l}\text { Garden, park, } \\
\text { national park/ } \\
\text { backyard } \\
(294,9.4 \%)\end{array}$ & $\begin{array}{c}\text { Oval/field } \\
(161,13.5 \%)\end{array}$ & 1128 \\
\hline 3 & $\begin{array}{l}\text { Living, family, } \\
\text { rumpus room } \\
(83,11.9 \%)\end{array}$ & $\begin{array}{c}\text { Other, interior } \\
(358,10.2 \%)\end{array}$ & $\begin{array}{l}\text { Garden, park, } \\
\text { national park/ } \\
\text { backyard } \\
(173,7.9 \%)\end{array}$ & $\begin{array}{c}\text { Other, exterior } \\
(226,7.2 \%)\end{array}$ & $\begin{array}{c}\text { Other, exterior } \\
(159,13.3 \%\end{array}$ & 999 \\
\hline 4 & $\begin{array}{c}\text { Other, interior } \\
(78,11.2 \%)\end{array}$ & $\begin{array}{c}\text { Bedroom } \\
(308,8.8 \%)\end{array}$ & $\begin{array}{c}\text { Other, interior } \\
(165,7.5 \%)\end{array}$ & $\begin{array}{c}\text { Playground with } \\
\text { or without play } \\
\text { equipment } \\
(207,6.6 \%)\end{array}$ & $\begin{array}{c}\text { Garden, park, } \\
\text { national park/ } \\
\text { backyard } \\
(98,8.2 \%)\end{array}$ & 856 \\
\hline 5 & $\begin{array}{l}\text { Kitchen } \\
(45,6.5 \%)\end{array}$ & $\begin{array}{c}\text { Garden, park, } \\
\text { national park/ } \\
\text { backyard } \\
(211,6 \%)\end{array}$ & $\begin{array}{l}\text { Living, family, } \\
\text { rumpus room } \\
(162,7.4 \%)\end{array}$ & $\begin{array}{l}\text { Bedroom } \\
(150,4.8 \%)\end{array}$ & $\begin{array}{c}\text { Playground with } \\
\text { or without play } \\
\text { equipment } \\
(51,4.3 \%)\end{array}$ & 619 \\
\hline 6 & $\begin{array}{l}\text { Bathroom } \\
(30,4.3 \%)\end{array}$ & $\begin{array}{l}\text { Bathroom } \\
(191,5.4 \%)\end{array}$ & $\begin{array}{c}\text { Playground with } \\
\text { or without play } \\
\text { equipment } \\
(148,6.7 \%)\end{array}$ & $\begin{array}{l}\text { Other, interior } \\
(133,4.3 \%)\end{array}$ & $\begin{array}{l}\text { Other, interior } \\
(50,4.2 \%)\end{array}$ & 552 \\
\hline 7 & $\begin{array}{c}\text { Stairs } \\
(29,4.2 \%)\end{array}$ & $\begin{array}{c}\text { Stairs } \\
(162,4.6 \%)\end{array}$ & $\begin{array}{c}\text { Other, exterior } \\
(89,4 \%)\end{array}$ & $\begin{array}{c}\text { Oval/field } \\
(113,3.6 \%)\end{array}$ & $\begin{array}{l}\text { Swimming pool } \\
(36,3 \%)\end{array}$ & 429 \\
\hline 8 & $\begin{array}{c}\text { Garden, park, } \\
\text { national park/ } \\
\text { backyard } \\
(18,2.6 \%)\end{array}$ & $\begin{array}{c}\text { Kitchen } \\
(149,4.2 \%)\end{array}$ & $\begin{array}{l}\text { Bathroom } \\
(85,3.9 \%)\end{array}$ & $\begin{array}{l}\text { Living, family, } \\
\text { rumpus room } \\
(112,3.6 \%)\end{array}$ & $\begin{array}{l}\text { Roadway, sealed } \\
\text { or unsealed } \\
\quad(36,3 \%)\end{array}$ & 400 \\
\hline 9 & $\begin{array}{l}\text { Car park/ } \\
\text { Driveway } \\
(9,1.3 \%)\end{array}$ & $\begin{array}{l}\text { Playground with } \\
\text { or without play } \\
\text { equipment } \\
(127,3.6 \%)\end{array}$ & $\begin{array}{c}\text { Stairs } \\
(66,3 \%)\end{array}$ & $\begin{array}{c}\text { Swimming pool } \\
(94,3 \%)\end{array}$ & $\begin{array}{c}\text { Court (tennis, } \\
\text { squash, etc.) } \\
(35,2.9 \%)\end{array}$ & 331 \\
\hline
\end{tabular}


Table XV. Cd.

\begin{tabular}{|c|c|c|c|c|c|c|}
\hline Ranking & $\begin{array}{c}\text { Infants (0 years) } \\
n=695\end{array}$ & $\begin{array}{c}1-2 \text { years age } \\
\text { group } \\
n=3511\end{array}$ & $\begin{array}{l}\text { 3-4 years } \\
\text { age group } \\
n=2201\end{array}$ & $\begin{array}{c}\text { 5-9 years age } \\
\text { group } \\
n=3120\end{array}$ & $\begin{array}{c}10-12 \text { years age } \\
\text { group } \\
n=1196\end{array}$ & $\begin{array}{c}\text { Total } \\
10723\end{array}$ \\
\hline 10 & $\begin{array}{c}\text { Other, exterior } \\
(7,1 \%)\end{array}$ & $\begin{array}{c}\text { Other, exterior } \\
(95,2.7 \%)\end{array}$ & $\begin{array}{c}\text { Kitchen } \\
(52,2.4 \%)\end{array}$ & $\begin{array}{l}\text { Roadway, sealed } \\
\text { or unsealed } \\
(73,2.3 \%)\end{array}$ & $\begin{array}{l}\text { Living, family, } \\
\text { rumpus room } \\
(25,2.1 \%)\end{array}$ & 252 \\
\hline 11 & $\begin{array}{l}\text { Roadway, sealed } \\
\text { or unsealed } \\
(4,0.6 \%)\end{array}$ & $\begin{array}{l}\text { Car park/ } \\
\text { Driveway } \\
(33,0.9 \%)\end{array}$ & $\begin{array}{l}\text { Car park/ } \\
\text { Driveway } \\
(44,2 \%)\end{array}$ & $\begin{array}{c}\text { Stairs } \\
(70,2.2 \%)\end{array}$ & $\begin{array}{l}\text { Classroom } \\
(21,1.8 \%)\end{array}$ & 172 \\
\hline 12 & $\begin{array}{c}\text { Footpath/path/ } \\
\text { foot track } \\
(4,0.6 \%)\end{array}$ & $\begin{array}{c}\text { Veranda or } \\
\text { balcony, ramp } \\
(31,0.9 \%)\end{array}$ & $\begin{array}{c}\text { Swimming pool } \\
(31,1.4 \%)\end{array}$ & $\begin{array}{l}\text { Car park/ } \\
\text { Driveway } \\
(69,2.2 \%)\end{array}$ & $\begin{array}{c}\text { Footpath/path/ } \\
\text { foot track } \\
(19,1.6 \%)\end{array}$ & 154 \\
\hline 13 & $\begin{array}{c}\text { Veranda or } \\
\text { balcony, ramp } \\
(3,0.4 \%)\end{array}$ & $\begin{array}{c}\text { Roadway, sealed } \\
\text { or unsealed } \\
920,0.6 \%)\end{array}$ & $\begin{array}{c}\text { Footpath/path/ } \\
\text { foot track } \\
(30,1.4 \%)\end{array}$ & $\begin{array}{c}\text { Classroom } \\
(63,2 \%)\end{array}$ & $\begin{array}{l}\text { Bedroom } \\
(17,1.4 \%)\end{array}$ & 133 \\
\hline 14 & $\begin{array}{l}\text { Laundry } \\
(1,0.1 \%)\end{array}$ & $\begin{array}{c}\text { Swimming pool } \\
(19,0.5 \%)\end{array}$ & $\begin{array}{l}\text { Roadway, sealed } \\
\text { or unsealed } \\
(25,1.1 \%)\end{array}$ & $\begin{array}{c}\text { Footpath/path/ } \\
\text { foot track } \\
(47,1.5 \%)\end{array}$ & $\begin{array}{c}\text { Skate park/ice } \\
\text { rink } \\
(16,0.5 \%)\end{array}$ & 108 \\
\hline 15 & $\begin{array}{c}\text { Gymnasium } \\
(1,0.1 \%)\end{array}$ & $\begin{array}{c}\text { Footpath/path/ } \\
\text { foot track } \\
(17,0.5 \%)\end{array}$ & $\begin{array}{c}\text { Veranda or } \\
\text { balcony, ramp } \\
(19,0.9 \%)\end{array}$ & $\begin{array}{l}\text { Kitchen } \\
(46,1.5 \%)\end{array}$ & $\begin{array}{l}\text { Kitchen } \\
(12,1 \%)\end{array}$ & 95 \\
\hline 16 & $\begin{array}{l}\text { Playground with } \\
\text { or without play } \\
\text { equipment } \\
(1,0.1 \%)\end{array}$ & $\begin{array}{c}\text { Miscellaneous } \\
(15,0.4 \%)\end{array}$ & $\begin{array}{c}\text { Toilet } \\
(9,0.4 \%)\end{array}$ & $\begin{array}{l}\text { Bathroom } \\
(39,1.3 \%)\end{array}$ & $\begin{array}{l}\text { Car park/ } \\
\text { Driveway } \\
(9,0.8 \%)\end{array}$ & 73 \\
\hline 17 & $\begin{array}{l}\text { Oval/field } \\
(1,0.1 \%)\end{array}$ & $\begin{array}{l}\text { Hall foyer } \\
(13,0.4 \%)\end{array}$ & $\begin{array}{c}\text { Classroom } \\
(7,0.3 \%)\end{array}$ & $\begin{array}{l}\text { Court (tennis, } \\
\text { squash, etc.) } \\
(21,0.7 \%)\end{array}$ & $\begin{array}{l}\text { Bathroom } \\
(9,0.8 \%)\end{array}$ & 51 \\
\hline 18 & $\begin{array}{c}\text { Beach } \\
(1,0.1 \%)\end{array}$ & $\begin{array}{l}\text { Classroom } \\
(12,0.3 \%)\end{array}$ & $\begin{array}{l}\text { Hall foyer } \\
(6,0.3 \%)\end{array}$ & $\begin{array}{c}\text { Paddock/field/ } \\
\text { camping ground } \\
(19,6 \%)\end{array}$ & $\begin{array}{c}\text { Stairs } \\
(8,0.7 \%)\end{array}$ & 46 \\
\hline 19 & $\begin{array}{l}\text { Classroom } \\
(0,0 \%)\end{array}$ & $\begin{array}{c}\text { Garage, } \\
\text { workshop, shed } \\
(8,0.2 \%)\end{array}$ & $\begin{array}{c}\text { Garage, } \\
\text { workshop, shed } \\
(6,0.3 \%)\end{array}$ & $\begin{array}{c}\text { Skate park/ } \\
\text { ice rink } \\
(16,0.5 \%)\end{array}$ & $\begin{array}{l}\text { Paddock/field/ } \\
\text { camping ground } \\
(8,0.7 \%)\end{array}$ & 38 \\
\hline 20 & $\begin{array}{c}\text { Miscellaneous } \\
(0,0 \%)\end{array}$ & $\begin{array}{l}\text { Paddock/field/ } \\
\text { camping ground } \\
(7,0.2 \%)\end{array}$ & $\begin{array}{l}\text { Paddock/field/ } \\
\text { camping ground } \\
(6,0.3 \%)\end{array}$ & $\begin{array}{c}\text { Miscellaneous } \\
(15,0.5 \%)\end{array}$ & $\begin{array}{l}\text { Beach } \\
(6,0.5 \%)\end{array}$ & 34 \\
\hline 21 & $\begin{array}{l}\text { Hall foyer } \\
(0,0 \%)\end{array}$ & $\begin{array}{l}\text { Area beneath } \\
\text { a building or } \\
\text { structure } \\
(5,0.1 \%)\end{array}$ & $\begin{array}{l}\text { Skate park/ } \\
\text { ice rink } \\
(5,0.2 \%)\end{array}$ & $\begin{array}{l}\text { Area beneath } \\
\text { a building or } \\
\text { structure } \\
(13,0.4 \%)\end{array}$ & $\begin{array}{l}\text { Hall foyer } \\
(5,0.4 \%)\end{array}$ & 28 \\
\hline 22 & $\begin{array}{l}\text { Toilet } \\
(0,0 \%)\end{array}$ & $\begin{array}{c}\text { Toilet } \\
(3,0.1 \%)\end{array}$ & $\begin{array}{c}\text { Miscellaneous } \\
(5,0.2 \%)\end{array}$ & $\begin{array}{c}\text { River/creek/lake/ } \\
\text { reservoir } \\
(13,0.4 \%)\end{array}$ & $\begin{array}{c}\text { River/creek/lake/ } \\
\text { reservoir } \\
(4,0.3 \%)\end{array}$ & 25 \\
\hline 23 & $\begin{array}{c}\text { Garage, } \\
\text { workshop, shed } \\
(0,0 \%)\end{array}$ & $\begin{array}{c}\text { Beach } \\
(3,0.1 \%)\end{array}$ & $\begin{array}{l}\text { Oval/field } \\
(3,0.1 \%)\end{array}$ & $\begin{array}{c}\text { Garage, } \\
\text { workshop, shed } \\
(13,0.4 \%)\end{array}$ & $\begin{array}{c}\text { Veranda or } \\
\text { balcony, ramp } \\
(4,0.3 \%)\end{array}$ & 23 \\
\hline 24 & $\begin{array}{l}\text { Court (tennis, } \\
\text { squash, etc.) } \\
(0,0 \%)\end{array}$ & $\begin{array}{l}\text { Laundry } \\
(2,0.1 \%)\end{array}$ & $\begin{array}{l}\text { Gymnasium } \\
(2,0.1 \%)\end{array}$ & $\begin{array}{c}\text { Beach } \\
(10,0.3 \%)\end{array}$ & $\begin{array}{c}\text { Area beneath } \\
\text { a building or } \\
\text { structure }(4,0.3 \%)\end{array}$ & 18 \\
\hline 25 & $\begin{array}{l}\text { Area beneath } \\
\text { a building or } \\
\text { structure }(0,0 \%)\end{array}$ & $\begin{array}{l}\text { Gymnasium } \\
(0,0 \%)\end{array}$ & $\begin{array}{l}\text { Laundry } \\
(1,0 \%)\end{array}$ & $\begin{array}{c}\text { Veranda or } \\
\text { balcony, ramp } \\
(10,0.3 \%)\end{array}$ & $\begin{array}{c}\text { Gymnasium } \\
(4,0.3 \%)\end{array}$ & 15 \\
\hline 26 & $\begin{array}{l}\text { Paddock/field/ } \\
\text { camping ground } \\
(0,0 \%)\end{array}$ & $\begin{array}{l}\text { Court (tennis, } \\
\text { squash, etc.) } \\
(0,0 \%)\end{array}$ & $\begin{array}{l}\text { Court (tennis, } \\
\text { squash, etc.) } \\
(1,0 \%)\end{array}$ & $\begin{array}{l}\text { Hall foyer } \\
(9,0.3 \%)\end{array}$ & $\begin{array}{c}\text { Sea/surf/bay/ } \\
\text { ocean } \\
(3,0.3 \%)\end{array}$ & 13 \\
\hline 27 & $\begin{array}{c}\text { Skate park/ice } \\
\text { rink } \\
(0,0 \%)\end{array}$ & $\begin{array}{c}\text { Skate park/ice } \\
\text { rink } \\
(0,0 \%)\end{array}$ & $\begin{array}{l}\text { River/creek/lake/ } \\
\text { reservoir } \\
(1,0 \%)\end{array}$ & $\begin{array}{c}\text { Gymnasium } \\
(9,0.3 \%)\end{array}$ & $\begin{array}{c}\text { Miscellaneous } \\
(3,0.3 \%)\end{array}$ & 13 \\
\hline
\end{tabular}


Table XV. Cd.

\begin{tabular}{|c|c|c|c|c|c|c|}
\hline Ranking & $\begin{array}{c}\text { Infants (0 years) } \\
n=695\end{array}$ & $\begin{array}{c}1-2 \text { years age } \\
\text { group } \\
n=3511\end{array}$ & $\begin{array}{c}3-4 \text { years } \\
\text { age group } \\
n=2201\end{array}$ & $\begin{array}{c}\text { 5-9 years age } \\
\text { group } \\
n=3120\end{array}$ & $\begin{array}{c}10-12 \text { years age } \\
\text { group } \\
n=1196\end{array}$ & $\begin{array}{c}\text { Total } \\
10723\end{array}$ \\
\hline 28 & $\begin{array}{c}\text { River/creek/lake/ } \\
\text { reservoir } \\
(0,0 \%)\end{array}$ & $\begin{array}{c}\text { Oval/field } \\
(0,0 \%)\end{array}$ & $\begin{array}{l}\text { Beach } \\
(1,0 \%)\end{array}$ & $\begin{array}{c}\text { Toilet } \\
(7,0.2 \%)\end{array}$ & $\begin{array}{c}\text { Garage, } \\
\text { workshop, shed } \\
(2,0.2 \%)\end{array}$ & 10 \\
\hline 29 & $\begin{array}{c}\text { Sea/surf/bay/ } \\
\text { ocean } \\
(0,0 \%)\end{array}$ & $\begin{array}{c}\text { River/creek/lake/ } \\
\text { reservoir } \\
(0,0 \%)\end{array}$ & $\begin{array}{l}\text { Area beneath } \\
\text { a building or } \\
\text { structure } \\
(0,0 \%)\end{array}$ & $\begin{array}{c}\text { Sea/surf/bay/ } \\
\text { ocean } \\
(7,0.2 \%)\end{array}$ & $\begin{array}{c}\text { Toilet } \\
(1,0.1 \%)\end{array}$ & 8 \\
\hline 30 & $\begin{array}{l}\text { Swimming pool } \\
(0,0 \%)\end{array}$ & $\begin{array}{c}\text { Sea/surf/bay/ } \\
\text { ocean } \\
(0,0 \%)\end{array}$ & $\begin{array}{c}\text { Sea/surf/bay/ } \\
\text { ocean } \\
(0,0 \%)\end{array}$ & $\begin{array}{c}\text { Laundry } \\
(1,0 \%)\end{array}$ & $\begin{array}{c}\text { Laundry } \\
(0,0 \%)\end{array}$ & 1 \\
\hline
\end{tabular}

Table XVI. Activities when injuries occurred, $p<0.001$

\begin{tabular}{|c|c|c|c|c|c|c|}
\hline Ranking & $\begin{array}{c}<1 \text { year age } \\
\text { group } \\
n=695\end{array}$ & $\begin{array}{c}1-2 \text { years age } \\
\text { group } \\
n=3511\end{array}$ & $\begin{array}{c}3-4 \text { years } \\
\text { age group } \\
n=2201\end{array}$ & $\begin{array}{c}5-9 \text { years age } \\
\text { group } \\
n=3120\end{array}$ & $\begin{array}{c}10-12 \text { years age } \\
\text { group } \\
n=1196\end{array}$ & $\begin{array}{c}\text { Total } \\
10723\end{array}$ \\
\hline 1 & $\begin{array}{l}\text { Being nursed or } \\
\text { care for } \\
(330,47.5 \%)\end{array}$ & $\begin{array}{c}\text { Playing } \\
(1423,40.5 \%)\end{array}$ & $\begin{array}{c}\text { Playing } \\
(1006,54.7 \%)\end{array}$ & $\begin{array}{c}\text { Playing } \\
(1020,32.7 \%)\end{array}$ & $\begin{array}{c}\text { Playing } \\
244,20.4 \%)\end{array}$ & 4023 \\
\hline 2 & $\begin{array}{c}\text { Resting. } \\
\text { Sleeping, eating, } \\
\text { other } \\
(130,18.7 \%)\end{array}$ & $\begin{array}{c}\text { Resting. } \\
\text { Sleeping, eating, } \\
\text { other } \\
9670,19.1 \%)\end{array}$ & $\begin{array}{c}\text { Resting. } \\
\text { Sleeping, eating, } \\
\text { other } \\
(375,17 \%)\end{array}$ & $\begin{array}{l}\text { Unspecified } \\
\text { activity } \\
(453,14.5 \%)\end{array}$ & $\begin{array}{l}\text { Unspecified } \\
\quad \text { activity } \\
(161,13.5 \%)\end{array}$ & 1789 \\
\hline 3 & $\begin{array}{c}\text { Playing } \\
(116,16.7 \%)\end{array}$ & $\begin{array}{l}\text { Being nursed or } \\
\text { care for } \\
(501,14.3 \%)\end{array}$ & $\begin{array}{l}\text { Unspecified } \\
\text { activity } \\
(296,13.4 \%)\end{array}$ & $\begin{array}{c}\text { Other specified } \\
\text { activity } \\
(355,11.4 \%)\end{array}$ & $\begin{array}{l}\text { Engaged in } \\
\text { formal education } \\
(150,12.5 \%)\end{array}$ & 1418 \\
\hline 4 & $\begin{array}{l}\text { Unspecified } \\
\text { activity } \\
(72,10.4 \%)\end{array}$ & $\begin{array}{l}\text { Unspecified } \\
\text { activity } \\
(470,13.4 \%)\end{array}$ & $\begin{array}{l}\text { Other specified } \\
\text { activity } \\
(199,9 \%)\end{array}$ & $\begin{array}{c}\text { Engaged in } \\
\text { formal education } \\
(343,11 \%)\end{array}$ & $\begin{array}{c}\text { Football, rugby, } \\
\text { soccer } \\
(139,11.6 \%)\end{array}$ & 1223 \\
\hline 5 & $\begin{array}{c}\text { Other specified } \\
\text { activity } \\
(45,6.5 \%)\end{array}$ & $\begin{array}{c}\text { Other specified } \\
\text { activity } \\
(347,9.9 \%)\end{array}$ & $\begin{array}{l}\text { Being nursed or } \\
\text { care for } \\
(117,5.3 \%)\end{array}$ & $\begin{array}{l}\text { Resting. sleeping, } \\
\text { eating, other } \\
(321,10.3 \%)\end{array}$ & $\begin{array}{c}\text { Other specified } \\
\text { activity } \\
(137,11.5 \%)\end{array}$ & 967 \\
\hline 6 & $\begin{array}{l}\text { Bicycling } \\
(1,0.1 \%)\end{array}$ & $\begin{array}{c}\text { Trampolining } \\
(29,0.8 \%)\end{array}$ & $\begin{array}{l}\text { Bicycling } \\
(67,3 \%)\end{array}$ & $\begin{array}{c}\text { Bicycling } \\
(128,4.1 \%)\end{array}$ & $\begin{array}{l}\text { Resting. sleeping, } \\
\text { eating, other } \\
(87,7.3 \%)\end{array}$ & 312 \\
\hline 7 & $\begin{array}{c}\text { Trampolining } \\
(1,0.1 \%)\end{array}$ & $\begin{array}{l}\text { Bicycling } \\
(22,0.6 \%)\end{array}$ & $\begin{array}{c}\text { Roller blading, } \\
\text { skateboarding } \\
(55,2.5 \%)\end{array}$ & $\begin{array}{c}\text { Roller blading, } \\
\text { skateboarding } \\
(124,4 \%)\end{array}$ & $\begin{array}{c}\text { Roller blading, } \\
\text { skateboarding } \\
(77,6.4 \%)\end{array}$ & 279 \\
\hline 8 & $\begin{array}{c}\text { Football, rugby, } \\
\text { soccer } \\
(0,0 \%)\end{array}$ & $\begin{array}{l}\text { Roller blading, } \\
\text { skateboarding } \\
(16,0.5 \%)\end{array}$ & $\begin{array}{l}\text { Trampolining } \\
(28,1.3 \%)\end{array}$ & $\begin{array}{l}\text { Swimming } \\
(89,2.9 \%)\end{array}$ & $\begin{array}{c}\text { Ball sport with } \\
\text { racquet, bat } \\
\text { (baseball, cricket, } \\
\text { hockey } \\
(53,4.4 \%)\end{array}$ & 186 \\
\hline 9 & $\begin{array}{l}\text { Jogging, walking } \\
(0,0 \%)\end{array}$ & $\begin{array}{l}\text { Swimming } \\
(14,0.4 \%)\end{array}$ & $\begin{array}{l}\text { Swimming } \\
(26,1.2 \%)\end{array}$ & $\begin{array}{c}\text { Football, rugby, } \\
\text { soccer } \\
(76,2.4 \%) \\
\end{array}$ & $\begin{array}{l}\text { Bicycling } \\
(44,3.7 \%)\end{array}$ & 160 \\
\hline 10 & $\begin{array}{c}\text { Gymnastics and } \\
\text { martial arts } \\
(0,0 \%)\end{array}$ & $\begin{array}{c}\text { Engaged in } \\
\text { formal education } \\
(6,0.2 \%)\end{array}$ & $\begin{array}{l}\text { Gymnastics and } \\
\text { martial arts } \\
(10,0.5 \%)\end{array}$ & $\begin{array}{c}\text { Trampolining } \\
(63,2 \%)\end{array}$ & $\begin{array}{l}\text { Swimming } \\
(33,2.8 \%)\end{array}$ & 112 \\
\hline 11 & $\begin{array}{l}\text { Ball sport with } \\
\text { racquet, bat } \\
\text { (baseball, cricket, } \\
\text { hockey) } \\
(0,0 \%)\end{array}$ & $\begin{array}{l}\text { Gymnastics and } \\
\text { Martial arts } \\
(5,0.1 \%)\end{array}$ & $\begin{array}{c}\text { Engaged in } \\
\text { formal education } \\
(8,0.4 \%)\end{array}$ & $\begin{array}{l}\text { Ball sport with } \\
\text { racquet, bat } \\
\text { (baseball, cricket, } \\
\text { hockey) } \\
(40,1.3 \%)\end{array}$ & $\begin{array}{l}\text { Basketball, } \\
\text { netball } \\
(22,1.8 \%)\end{array}$ & 75 \\
\hline
\end{tabular}


Table XVI. Cd.

\begin{tabular}{|c|c|c|c|c|c|c|}
\hline Ranking & $\begin{array}{c}<1 \text { year age } \\
\text { group } \\
n=695\end{array}$ & $\begin{array}{c}1-2 \text { years age } \\
\text { group } \\
n=3511\end{array}$ & $\begin{array}{l}3-4 \text { years } \\
\text { age group } \\
n=2201\end{array}$ & $\begin{array}{c}\text { 5-9 years age } \\
\text { group } \\
n=3120\end{array}$ & $\begin{array}{c}\text { 10-12 years age } \\
\text { group } \\
n=1196\end{array}$ & $\begin{array}{r}\text { Total } \\
10723\end{array}$ \\
\hline 12 & $\begin{array}{c}\text { Motorised } \\
\text { sports (go-kart, } \\
\text { minibike, motor } \\
\text { cross) } \\
(0,0 \%)\end{array}$ & $\begin{array}{c}\text { Jogging, Walking } \\
\qquad(4,0.1 \%)\end{array}$ & $\begin{array}{l}\text { Ball sport with } \\
\text { racquet, bat } \\
\text { (baseball, cricket, } \\
\text { hockey) } \\
(6,0.3 \%)\end{array}$ & $\begin{array}{l}\text { Being nursed or } \\
\text { care for } \\
(32,1 \%)\end{array}$ & $\begin{array}{c}\text { Trampolining } \\
(20,1.7 \%)\end{array}$ & 62 \\
\hline 13 & $\begin{array}{c}\text { Basketball, } \\
\text { netball } \\
(0,0 \%)\end{array}$ & $\begin{array}{c}\text { Motorised } \\
\text { sports (go-kart, } \\
\text { minibike, motor } \\
\text { cross) } \\
(3,0.1 \%)\end{array}$ & $\begin{array}{c}\text { Motorised } \\
\text { sports (go-kart, } \\
\text { minibike, motor } \\
\text { cross) } \\
(3,0.1 \%)\end{array}$ & $\begin{array}{c}\text { Motorised } \\
\text { sports (go-kart, } \\
\text { minibike, motor } \\
\text { cross) } \\
(21,0.7 \%)\end{array}$ & $\begin{array}{l}\text { Being nursed or } \\
\text { care for } \\
(8,0.7 \%)\end{array}$ & 35 \\
\hline 14 & $\begin{array}{c}\text { Roller blading, } \\
\text { skateboarding } \\
(0,0 \%)\end{array}$ & $\begin{array}{c}\text { Fishing and } \\
\text { boating } \\
(1,0 \%)\end{array}$ & $\begin{array}{c}\text { Basketball, } \\
\text { netball } \\
(2,0.1 \%)\end{array}$ & $\begin{array}{c}\text { Gymnastics and } \\
\text { martial arts } \\
(18,0.6 \%)\end{array}$ & $\begin{array}{c}\text { Gymnastics and } \\
\text { martial arts } \\
(7,0.6 \%)\end{array}$ & 28 \\
\hline 15 & $\begin{array}{l}\text { Horse riding } \\
(0,0 \%)\end{array}$ & $\begin{array}{l}\text { Ball sport with } \\
\text { racquet, bat } \\
\text { (baseball, cricket, } \\
\text { hockey) } \\
(0,0 \%)\end{array}$ & $\begin{array}{l}\text { Football, rugby, } \\
\text { soccer } \\
(1,0 \%)\end{array}$ & $\begin{array}{l}\text { Fishing and } \\
\text { boating } \\
(16,0.5 \%)\end{array}$ & $\begin{array}{l}\text { Horse riding } \\
(6,0.5 \%)\end{array}$ & 23 \\
\hline 16 & $\begin{array}{c}\text { Fishing and } \\
\text { boating } \\
(0,0 \%)\end{array}$ & $\begin{array}{c}\text { Basketball, } \\
\text { netball } \\
(0,0 \%)\end{array}$ & $\begin{array}{l}\text { Horse riding } \\
\quad(1,0 \%)\end{array}$ & $\begin{array}{c}\text { Basketball, } \\
\text { netball } \\
(7,0.2 \%)\end{array}$ & $\begin{array}{c}\text { Fishing and } \\
\text { boating } \\
(3,0.3 \%)\end{array}$ & 11 \\
\hline 17 & $\begin{array}{l}\text { Swimming } \\
(0,0 \%)\end{array}$ & $\begin{array}{l}\text { Football, rugby, } \\
\text { soccer } \\
(0,0 \%)\end{array}$ & $\begin{array}{c}\text { Fishing and } \\
\text { boating } \\
(1,0 \%)\end{array}$ & $\begin{array}{l}\text { Horse riding } \\
(7,0.2 \%)\end{array}$ & $\begin{array}{c}\text { Motorised } \\
\text { sports (go-kart, } \\
\text { minibike, motor } \\
\text { cross) } \\
(3,0.3 \%)\end{array}$ & 11 \\
\hline 18 & $\begin{array}{l}\text { Water skiing } \\
\qquad(0,0 \%)\end{array}$ & $\begin{array}{l}\text { Horse riding } \\
\quad(0,0 \%)\end{array}$ & $\begin{array}{c}\text { Jogging, walking } \\
(0,0 \%)\end{array}$ & $\begin{array}{l}\text { Jogging, walking } \\
(4,0.1 \%)\end{array}$ & $\begin{array}{l}\text { Jogging, walking } \\
(1,0.1 \%)\end{array}$ & 5 \\
\hline 19 & $\begin{array}{c}\text { Engaged in } \\
\text { formal education } \\
(0,0 \%)\end{array}$ & $\begin{array}{c}\text { Water skiing } \\
(0,0 \%)\end{array}$ & $\begin{array}{c}\text { Water skiing } \\
(0,0 \%)\end{array}$ & $\begin{array}{c}\text { Water skiing } \\
(3,0.1 \%)\end{array}$ & $\begin{array}{c}\text { Water skiing } \\
\quad(1,0.1 \%)\end{array}$ & 4 \\
\hline
\end{tabular}

Table XVII. Nature of injury by age group

\begin{tabular}{|c|c|c|c|c|c|c|}
\hline Nature of injury & $\begin{array}{l}\text { Less than } 12 \\
\text { months }\end{array}$ & $1-2$ years & 3-4 years & 5-9 years & 10-12 years & Total \\
\hline $\begin{array}{l}\text { Open wound } \\
\text { (excludes eye) }\end{array}$ & $(165,23 \%)$ & $(1719,49.0 \%)$ & $(1138,51.7 \%)$ & $(1209,38.85)$ & $(296,24.7 \%)$ & $(4527,42.25)$ \\
\hline $\begin{array}{l}\text { Superficial (incl. bruise; } \\
\text { excl. eye) }\end{array}$ & $(414,59.6 \%)$ & $(1172,33.4 \%)$ & $(593,26.9 \%)$ & $(768,24.6 \%)$ & $(356,29.85)$ & $(3303,30.8 \%)$ \\
\hline $\begin{array}{l}\text { Eye injury (excl. foreign } \\
\text { body in external eye } \\
{[14.1] \text {; includes burn) }}\end{array}$ & $(59,8.5 \%)$ & $(378,10.8 \%)$ & $(310,14.15)$ & $(715,22.9 \%)$ & $(328,27.4 \%)$ & $(1790,16.7 \%)$ \\
\hline $\begin{array}{l}\text { Miscellaneous or } \\
\text { unspecified }\end{array}$ & $(11,1.6 \%)$ & $(32,0.9 \%)$ & $(44,2.0 \%)$ & $(167,5.4 \%)$ & $(59,4.9 \%)$ & $(313,2.9 \%)$ \\
\hline $\begin{array}{l}\text { Fracture and dislocation } \\
\text { (excludes tooth) }\end{array}$ & $(2,0.3 \%)$ & $(26,0.7 \%)$ & $(36,1.6 \%)$ & $(100,3.2 \%)$ & $(73,6.1 \%)$ & $(237,2.2 \%)$ \\
\hline $\begin{array}{l}\text { Dental injury (incl. } \\
\text { fractured tooth) }\end{array}$ & $(5,0.7 \%)$ & $(72,2.1 \%)$ & $(46,2.1 \%)$ & $(81,2.6 \%)$ & $(23,1.9 \%)$ & $(227,2.1 \%)$ \\
\hline $\begin{array}{l}\text { Burn or corrosion } \\
\text { (excl. eye) }\end{array}$ & $(27,3.9 \%)$ & $(89,2.5 \%)$ & $(13,0.6 \%)$ & $(28,0.9 \%)$ & $(19,1.6 \%)$ & $(176,1.6 \%)$ \\
\hline $\begin{array}{l}\text { Intracranial injury } \\
\text { (includes concussion) }\end{array}$ & $(10,1.4 \%)$ & $(14,0.4 \%)$ & $(12,0.5 \%)$ & $(27,0.9 \%)$ & $(31,2.6 \%)$ & $(94,0.9 \%)$ \\
\hline $\begin{array}{l}\text { Effect of venom; } \\
\text { any insect bite }\end{array}$ & $(2,0.3 \%)$ & $(9,0.3 \%)$ & $(9,0.4 \%)$ & $(25,0.8 \%)$ & $(11,0.9 \%)$ & $(56,0.5 \%)$ \\
\hline Total & $(695,100 \%)$ & $(3511,100 \%)$ & $(2201,100 \%)$ & $(3120,100 \%)$ & $(1196,100 \%)$ & $(10723,100 \%)$ \\
\hline
\end{tabular}


Table XVIII. $P$-values of variables

\begin{tabular}{|c|c|c|c|c|c|}
\hline Variables & Triage & Admission status & $\begin{array}{l}\text { Mechanism of } \\
\text { injury }\end{array}$ & $\begin{array}{l}\text { Body region } \\
\text { injured }\end{array}$ & Nature of injury \\
\hline $\begin{array}{l}\text { Mechanism of } \\
\text { injury }\end{array}$ & $\begin{array}{l}p<0.001 \\
\text { Test valid }\end{array}$ & $\begin{array}{c}p<0.001 \\
\chi^{2} \text { assumptions } \\
\text { not fulfilled }\end{array}$ & $x$ & $\begin{array}{c}p<0.001 \\
\chi^{2} \text { assumptions } \\
\text { not fulfilled }\end{array}$ & $\begin{array}{c}p<0.001 \\
\chi^{2} \text { assumptions } \\
\text { not fulfilled }\end{array}$ \\
\hline $\begin{array}{l}\text { Nature of } \\
\text { injury }\end{array}$ & $\begin{array}{l}p<0.001 \\
\text { Test valid }\end{array}$ & $\begin{array}{c}p<0.001 \\
\chi^{2} \text { assumptions } \\
\text { not fulfilled }\end{array}$ & $\begin{array}{c}p<0.001 \\
\chi^{2} \text { assumptions } \\
\text { not fulfilled }\end{array}$ & $\begin{array}{c}p<0.001 \\
\chi^{2} \text { assumptions } \\
\text { not fulfilled }\end{array}$ & $x$ \\
\hline $\begin{array}{l}\text { Body of } \\
\text { region }\end{array}$ & $\begin{array}{l}p<0.001 \\
\text { Test valid }\end{array}$ & $\begin{array}{l}p<0.001 \\
\text { Test valid }\end{array}$ & $\begin{array}{c}p<0.001 \\
\chi^{2} \text { assumptions } \\
\text { not fulfilled }\end{array}$ & $x$ & $\begin{array}{c}p<0.001 \\
\chi^{2} \text { assumptions } \\
\text { not fulfilled }\end{array}$ \\
\hline Activity & $\begin{array}{c}p<0.001 \\
\chi^{2} \text { assumptions } \\
\text { not fulfilled }\end{array}$ & $\begin{array}{c}p<0.001 \\
\chi^{2} \text { assumptions } \\
\text { not fulfilled }\end{array}$ & $\begin{array}{c}p<0.001 \\
\chi^{2} \text { assumptions } \\
\text { not fulfilled }\end{array}$ & $\begin{array}{c}p<0.001 \\
\chi^{2} \text { assumptions } \\
\text { not fulfilled }\end{array}$ & $\begin{array}{c}p<0.001 \\
\chi^{2} \text { assumptions } \\
\text { not fulfilled }\end{array}$ \\
\hline Type of place & $\begin{array}{l}p<0.001 \\
\text { Test valid }\end{array}$ & $\begin{array}{c}p<0.001 \\
\chi^{2} \text { assumptions } \\
\text { not fulfilled }\end{array}$ & $\begin{array}{c}p<0.001 \\
\chi^{2} \text { assumptions } \\
\text { not fulfilled }\end{array}$ & $\begin{array}{c}p<0.001 \\
\chi^{2} \text { assumptions } \\
\text { not fulfilled }\end{array}$ & $\begin{array}{c}p<0.001 \\
\chi^{2} \text { assumptions } \\
\text { not fulfilled }\end{array}$ \\
\hline Part of place & $\begin{array}{c}p<0.001 \\
\chi^{2} \text { assumptions } \\
\text { not fulfilled }\end{array}$ & $\begin{array}{c}p<0.001 \\
\chi^{2} \text { assumptions } \\
\text { not fulfilled }\end{array}$ & $\begin{array}{c}p<0.001 \\
\chi^{2} \text { assumptions } \\
\text { not fulfilled }\end{array}$ & $\begin{array}{c}p<0.001 \\
\chi^{2} \text { assumptions } \\
\text { not fulfilled }\end{array}$ & $\begin{array}{c}p<0.001 \\
\chi^{2} \text { assumptions } \\
\text { not fulfilled }\end{array}$ \\
\hline Time of day & $\begin{array}{l}p<0.049 \\
\text { Test valid }\end{array}$ & $\begin{array}{l}p<0.001 \\
\text { Test valid }\end{array}$ & $\begin{array}{l}p<0.001 \\
\text { Test valid }\end{array}$ & $\begin{array}{l}p<0.001 \\
\text { Test valid }\end{array}$ & $\begin{array}{l}p<0.001 \\
\text { Test valid }\end{array}$ \\
\hline Day of week & $p=0.784$ & $p=0.655$ & $p=0.044$ & $p=0.560$ & $\begin{array}{l}p<0.001 \\
\text { Test valid }\end{array}$ \\
\hline Season & $p=0.021$ & $p=0.891$ & $\begin{array}{l}p<0.001 \\
\text { Test valid }\end{array}$ & $p=0.333$ & $p=0.311$ \\
\hline $\begin{array}{l}\text { Indigenous } \\
\text { status }\end{array}$ & $p=0.308$ & $\begin{array}{l}p<0.001 \\
\text { Test valid }\end{array}$ & $\begin{array}{l}p=0.030 \\
\text { Test valid }\end{array}$ & $\begin{array}{l}p=0.002 \\
\text { Test valid }\end{array}$ & $p=0.062$ \\
\hline Gender & $p=0.110$ & $p=0.539$ & $\begin{array}{l}p<0.001 \\
\text { Test valid }\end{array}$ & $p=0.073$ & $\begin{array}{l}p<0.001 \\
\text { Test valid }\end{array}$ \\
\hline
\end{tabular}

\section{Other associations between injury} and event characteristics

As shown in Table XVIII, the associations between injury and event characteristics were different; some relations were significant, but others were not.

\section{Injury and event characteristics by indigenous status}

Associations between age, gender, and Indigenous status

There was not a significant association between indigenous status and age group ( $p=$ 0.111 ), and the association between indigenous status and gender approached significance $(p=0.050)$. Table XIX shows the relationship between indigenous status and other variables in the study.

Mechanism of injury by indigenous status

Mechanism of injury was significantly associated with indigenous status $\left(\chi^{2}=26.89, \mathrm{~d} f=15\right.$, $p=0.030)$. As shown in Table $\mathrm{XX}$, 'contact with a static object' followed by 'cutting and tearing' were the leading mechanisms in both indigenous and non-indigenous cases. The third most common mechanism was 'stumbling, tripping on the same level' for Indigenous children and 'other fall' for non-Indigenous children.

Body region of injury by indigenous status

There was an association between body region of injury and indigenous status $\left(\chi^{2}=14.72, \mathrm{~d} f=3\right.$,

Table XIX. The associations of indigenous status with other variables

\begin{tabular}{|lc|}
\hline Triage & $p=0.308$ \\
\hline Mechanism of injury & $p=0.030$ \\
\hline Nature of injury & $p=0.062$ \\
\hline Body of region & $p=0.002$ \\
\hline Admission status & $p<0.001$ \\
\hline Activity & Unable to conduct $\chi^{2}$ test \\
\hline Part of place & Unable to conduct $\chi^{2}$ test \\
\hline Type of place & $p<0.001$ \\
\hline Time of day & $p<0.001$ \\
\hline Day of week & $p=0.873$ \\
\hline Season & $p=0.602$ \\
\hline Gender & $p=0.050$ \\
\hline Age group & $p=0.111$ \\
\hline
\end{tabular}


Table XX. Mechanism of injury by indigenous status

\begin{tabular}{|c|c|c|c|}
\hline \multirow{2}{*}{$\begin{array}{l}\text { Mechanism of_injury } \\
p=0.030\end{array}$} & \multicolumn{2}{|c|}{ Indigenous status } & \multirow[t]{2}{*}{ Total } \\
\hline & $\begin{array}{l}\text { Aboriginal } \\
\text { and/or TSI }\end{array}$ & Not indigenous & \\
\hline Contact with static object & $152,19.6 \%$ & $2063,21 \%$ & $2215,20.9 \%$ \\
\hline Cutting and tearing & $103,13.3 \%$ & $1247,12.75$ & $1350,12.7 \%$ \\
\hline Other fall & $70,9 \%$ & $999,10.2 \%$ & $1069,10.1 \%$ \\
\hline Fall by stumbling, tripping on same level & $87,11.2 \%$ & $957,9.7 \%$ & $1044,9.8 \%$ \\
\hline Fall/jump from lesser height (less than $1 \mathrm{~m}$ ) & $70,9 \%$ & $900,9.2 \%$ & $970,9.1 \%$ \\
\hline Contact with moving object & $49,6.3 \%$ & $897,9.1 \%$ & $946,8.9 \%$ \\
\hline Contact with person & $74,9.5 \%$ & $814,8.3 \%$ & $888,8.4 \%$ \\
\hline Bite or sting by animal, human, insect & $42,5.4 \%$ & $333,3.4 \%$ & $375,3.5 \%$ \\
\hline Miscellaneous and unspecified mechanism of injury & $25,3.2 \%$ & $438,4.5 \%$ & $463,4.4 \%$ \\
\hline Fall on or from stairs & $24,3.1 \%$ & $266,2.7 \%$ & $290,2.7 \%$ \\
\hline Other, unspecified contact & $18,2.3 \%$ & $211,2.1 \%$ & $229,2.2 \%$ \\
\hline Burns & $13,1.7 \%$ & $181,1.8 \%$ & $194,1.8 \%$ \\
\hline Fall/jump from greater height (more than $1 \mathrm{~m}$ ) & $12,1.5 \%$ & $179,1.8 \%$ & $191,1.8 \%$ \\
\hline Contact with animal & $19,2.4 \%$ & $159,1.6 \%$ & $178,1.7 \%$ \\
\hline All pinch, crush, puncture, pierce & $15,1.9 \%$ & $146,1.5 \%$ & $161,1.5 \%$ \\
\hline Contact, splash, spill & $4,0.5 \%$ & $42,0.4 \%$ & $64,0.4 \%$ \\
\hline Total & $777,100 \%$ & $9832,100 \%$ & $10,609,100 \%$ \\
\hline
\end{tabular}

Table XXI. Body region injury by indigenous status

\begin{tabular}{|lccc|}
\hline \multirow{2}{*}{$\begin{array}{l}\text { Body regions } \\
\boldsymbol{p}=\mathbf{0 . 0 0 2}\end{array}$} & \multicolumn{2}{c}{ Indigenous status } & \multirow{2}{*}{ Total } \\
\cline { 2 - 3 } & Aboriginal and/or TSI & Not indigenous & \\
\hline Face (excludes eye) & $316,40.7 \%$ & $4021,40.9 \%$ & $4337,40.9 \%$ \\
\hline Head (excludes face) & $331,42.6 \%$ & $3713,37.8 \%$ & $4044,38.1 \%$ \\
\hline Body location not required & $115,14.8 \%$ & $1951,19.8 \%$ & $2066,19.5 \%$ \\
\hline $\begin{array}{l}\text { Multiple injuries (involving more } \\
\text { than one bodily location) }\end{array}$ & $15,14.8 \%$ & $147,1.5 \%$ & $162,1.5 \%$ \\
\hline Total & $777,100 \%$ & $9832,100 \%$ & $10,609,100 \%$ \\
\hline
\end{tabular}

Table XXII. Mode of separation by indigenous status

\begin{tabular}{|lccc|}
\hline $\begin{array}{l}\text { Outcome of ED visit } \\
\text { (Mode of separation) } \\
p<0.001\end{array}$ & \multicolumn{2}{c}{ Indigenous status } & Total \\
\cline { 2 - 3 } & Aboriginal and/or TSI & Not indigenous & \\
\hline $\begin{array}{l}\text { ED service event completed }- \\
\text { discharged }\end{array}$ & $667,85.8 \%$ & $8114,82.8 \%$ & $8811,83.1 \%$ \\
\hline $\begin{array}{l}\text { Admitted to same or other } \\
\text { hospital }\end{array}$ & $90,11.6 \%$ & $1556,15.8 \%$ & $1646,15.5 \%$ \\
\hline $\begin{array}{l}\text { Did not wait/left after treatment } \\
\text { commenced }\end{array}$ & $20,2.6 \%$ & $132,1.3 \%$ & $152,1.4 \%$ \\
\hline Total & $777,100 \%$ & $9832,100 \%$ & $10,609,100 \%$ \\
\hline
\end{tabular}

$p=0.002$ ). Table $X X \mid$ shows that the proportion of children with facial injuries was very similar for both indigenous and non-indigenous children (40\%). However, indigenous children had higher proportions of head injuries (42.6\%) and multiple injuries than non-indigenous children.
Mode of separation by indigenous status

There was an association between mode of separation and indigenous status $\left(\chi^{2}=16.76\right.$, $\mathrm{d} f=2, p<0.001)$. As shown in Table XXII, indigenous patients were more likely to have the ED service event completed and be discharged (85.8\%), 
Table XXIII. Type of place by indigenous status

\begin{tabular}{|lccc|}
\hline \multirow{2}{*}{$\begin{array}{l}\text { Type of place } \\
\boldsymbol{y}<0.001\end{array}$} & \multicolumn{2}{c}{ Indigenous status } & Total \\
\cline { 2 - 3 } & Aboriginal and/or TSI & Not indigenous & \\
\hline Home (includes farmhouse) & $537,69.1 \%$ & $6367,64.8 \%$ & $6904,65.1 \%$ \\
\hline Unspecified place & $93,12 \%$ & $731,7.4 \%$ & $824,7.8 \%$ \\
\hline Primary, secondary school & $30,3.9 \%$ & $707,7.2 \%$ & $737,6.9 \%$ \\
\hline Childcare, preschool centre & $14,1.8 \%$ & $412,4.2 \%$ & $426,4 \%$ \\
\hline Public park & $27,3.5 \%$ & $340,3.5 \%$ & $367,3.5 \%$ \\
\hline Oval, fields, pitch & $18,2.3 \%$ & $256,2.6 \%$ & $274,2.6 \%$ \\
\hline Street or highway (public road) & $16,2.1 \%$ & $219,2.2 \%$ & $235,2.2 \%$ \\
\hline Miscellaneous specified place & $11,1.4 \%$ & $217,2.2 \%$ & $228,2.1 \%$ \\
\hline $\begin{array}{l}\text { Recreation area (informal), specified, } \\
\text { incl. amusement park, aquatic } \\
\text { recreation centre }\end{array}$ & $12,1.5 \%$ & $215,2.2 \%$ & $227,2.1 \%$ \\
\hline $\begin{array}{l}\text { Trade or service area, incl. shopping } \\
\text { centre, restaurant }\end{array}$ & $10,1.3 \%$ & & \\
\hline Other and unspecified sports area & $1,0.1 \%$ & $187,1.9 \%$ & $197,1.95$ \\
\hline Farm (excl. farmhouse) & $1,0.1 \%$ & $77,0.8 \%$ & $78,0.7 \%$ \\
\hline Bush, remote or undeveloped place & $3,0.4 \%$ & $42,0.4 \%$ & $43,0.4 \%$ \\
\hline $\begin{array}{l}\text { Hospital, medical clinic, or other health } \\
\text { service }\end{array}$ & $4,0.5 \%$ & $39,0.4 \%$ & $42,0.4 \%$ \\
\hline Total & $777,100 \%$ & $23,0.2 \%$ & $27,0.3 \%$ \\
\hline
\end{tabular}

or to leave before completion of the event (2.6\%) Non-indigenous patients were more likely to be admitted to hospital (15.8\%).

Activity by indigenous status

There was a relationship between indigenous status and type of activity the injured child was engaged in when the injury occurred $\left(\chi^{2}=66.97\right.$, $\mathrm{d} f=18, p<0.001$, but $\chi^{2}$ assumptions were not fulfilled, and it was not possible to perform an exact test). Additionally, it was found that the highest frequency of injuries occurred when the children were playing, for both indigenous and non-indigenous children. Interestingly, there was a higher incidence of injury among children engaged in the activity of rollerblading/skateboarding in aboriginal and/or TSI than in children engaged in formal education (as student); whereas, the opposite was true with non-indigenous children.

Type of place by indigenous status

In this study, there was an association between indigenous status and type of place $\left(\chi^{2}=56.17\right.$, $\mathrm{d} f=13, p<0.001)$. As shown in Table XXIII, the primary areas where injury occurred were the home, followed by 'unspecified place', and then primary/secondary school, for both indigenous and non-indigenous children, but the proportion in each place varied by indigenous status.
Time of day by indigenous status

There was relationship between indigenous status and time of day of injury $\left(\chi^{2}=63.03, \mathrm{~d} f=5\right.$, $p<0.001)$. The greatest proportion of injuries occurred in the 3-hour time period of 3-6 p.m. for both indigenous and non-indigenous cases. More indigenous cases presented overnight from 9 p.m. -6 a.m., while less presented in the morning 6-9 a.m. and 9 a.m. - midday (Table XXIV).

\section{Gender by indigenous status}

The association between indigenous status and gender approached significance $\left(\chi^{2}=3.85\right.$, $\mathrm{d} f=1, p<0.050)$. As shown in the Table XXV below, males were more overrepresented among indigenous children (males 65.8\%) than among non-indigenous children (males 62.2\%).

Injury and event characteristic by time

Table XXVI demonstrates associations between time of day and other variables in the dataset.

\section{Triage by time of injury}

There was an association between triage (severity of the injury) and time of injury $\left(\chi^{2}=25.06\right.$, $\mathrm{d} f=15, p=0.049)$. As shown in the Table XXVII, semi urgent (60 min) was the most common triage for each coded temporal period in the present study. 
Table XXIV. Injury time by indigenous status

\begin{tabular}{|lccc|}
\hline \multirow{2}{*}{$\begin{array}{l}\text { Injury time } \\
\boldsymbol{p}<0.001\end{array}$} & \multicolumn{2}{c|}{ Indigenous status } & \multirow{2}{*}{ Total } \\
\cline { 2 - 3 } & Aboriginal and/or TSI & Not indigenous & \\
\hline 3-6 p.m. & $218,28.1 \%$ & $2764,28.1 \%$ & $2982,28.1 \%$ \\
\hline 6-9 p.m. & $165,21.2 \%$ & $2091,21.3 \%$ & $2256,21.3 \%$ \\
\hline Midday - 3 p.m. & $148,19 \%$ & $1739,17.7 \%$ & $1887,17.8 \%$ \\
\hline 9 a.m. - midday & $93,12 \%$ & $1689,17.2 \%$ & $1782,16.3 \%$ \\
\hline 9 p.m. -6 a.m. & $120,15.4 \%$ & $822,8.45$ & $942,8.9 \%$ \\
\hline 6-9 a.m. & $33,4.2 \%$ & $727,7.4 \%$ & $760,7.2 \%$ \\
\hline Total & $777,100 \%$ & $9832,100 \%$ & $10,609,100 \%$ \\
\hline
\end{tabular}

Table XXV. Gender by indigenous status

\begin{tabular}{|lccc|}
\hline \multirow{2}{*}{$\begin{array}{l}\text { Gender } \\
\boldsymbol{p}<0.001\end{array}$} & \multicolumn{2}{c}{ Indigenous status } & \multirow{2}{*}{ Total } \\
\cline { 2 - 3 } & Aboriginal and/or TSI & Not indigenous & \\
\hline Male & $511,65.8 \%$ & $6118,62.2 \%$ & $6629,62.5 \%$ \\
\hline Female & $266,34.2 \%$ & $3714,37.8 \%$ & $3980,37.5 \%$ \\
\hline Total & $777,100 \%$ & $9832,100 \%$ & $10,609,100 \%$ \\
\hline
\end{tabular}

Mechanism of injury by time

There was strong evidence of an association between the mechanism of injury and time of injury $\left(\chi^{2}=306.58, d f=75, p<0.001\right)$. The most frequent mechanism of injury was 'contact with a static object' at each coded temporal time of injury in this study, but this category was overrepresented at 6-9 a.m. and 6-9 p.m. Interestingly, more cases of injuries as result of 'fall/jump from lesser height (less than $1 \mathrm{~m}$ )' than cases occurred from 'fall by stumbling, tripping on same level' occurred at 6-9 a.m., but the opposite was true in the rest of temporal hours, as shown in the Table XXVIII.

Nature of injury by injury time

There was an association between nature of injury and time of injury $\left(\chi^{2}=306.58, \mathrm{~d} f=75\right.$, $p<0.001)$. Open wound was the predominant injury at all coded times of injury in this study, but it was most represented in the 6-9 p.m. time period. Interestingly, there were more burn or corrosion
Table XXVI. The associations of injury time (timeblock of day) with other variables

\begin{tabular}{|lc|}
\hline Triage & $p=0.049$ \\
\hline Mechanism of injury & $p<0.001$ \\
\hline Nature of injury & $p<0.001$ \\
\hline Body of region & $p<0.001$ \\
\hline Admission status & $p<0.001$ \\
\hline Activity & $\chi^{2}$ test not valid \\
\hline Part of place & $\chi^{2}$ test not valid \\
\hline Type of place & $p<0.001$ \\
\hline Gender & $p=0.007$ \\
\hline Age group & $p<0.001$ \\
\hline Day of week & $p<0.001$ \\
\hline Season & $p<0.001$ \\
\hline Month & $p<0.001$ \\
\hline
\end{tabular}

(excl. eye) cases occurred at 6-9 a.m. than miscellaneous or unspecified, fracture and dislocation (excludes tooth), and dental injury (incl. fractured

Table XXVII. Triage by time of injury

\begin{tabular}{|c|c|c|c|c|c|c|c|}
\hline \multirow{2}{*}{$\begin{array}{l}\text { Triage } \\
p=0.049\end{array}$} & \multicolumn{6}{|c|}{ Time of injury } & \multirow[t]{2}{*}{ Total } \\
\hline & 6-9 a.m. & $\begin{array}{l}9 \text { a.m. - } \\
\text { midday }\end{array}$ & $\begin{array}{l}\text { Midday - } \\
3 \text { p.m. }\end{array}$ & 3-6 p.m. & 8-9 p.m. & $\begin{array}{l}9 \text { p.m. - } \\
6 \text { a.m. }\end{array}$ & \\
\hline $\begin{array}{l}\text { Semi-urgent } \\
\text { (60 min) }\end{array}$ & $554,72.4 \%$ & $1199,66.8 \%$ & $1272,66.9 \%$ & $2032,67.4 \%$ & $1601,70.2 \%$ & $657,68.2 \%$ & $7315,68.2 \%$ \\
\hline $\begin{array}{l}\text { Urgent } \\
\text { (30 min) }\end{array}$ & $143,18.7 \%$ & $400,22.3 \%$ & $423,22.2 \%$ & $663,22 \%$ & $452,19.8 \%$ & $203,21.1 \%$ & $2284,21.3 \%$ \\
\hline Emergency & $44,5.85$ & $111,6.25$ & $111,5.8 \%$ & $172,5.7 \%$ & $103,4.5 \%$ & $52,5.4 \%$ & $593,5.5 \%$ \\
\hline $\begin{array}{l}\text { Non-urgent } \\
\text { (120 min) }\end{array}$ & $24,3.15$ & $84,4.7 \%$ & $96,5 \%$ & $149,4.9 \%$ & $126,5.5 \%$ & $51,5.3 \%$ & $530,4.9 \%$ \\
\hline Total & $765,100 \%$ & $1794,100 \%$ & $1902,100 \%$ & 3016,100 & $2282,100 \%$ & $963,100 \%$ & $10,722,100 \%$ \\
\hline
\end{tabular}


Table XXVIII. Mechanism of injury by time

\begin{tabular}{|c|c|c|c|c|c|c|c|}
\hline \multirow{2}{*}{$\begin{array}{l}\text { Mechanism of } \\
\text { injury } \\
p<0.001\end{array}$} & \multicolumn{6}{|c|}{ Time of injury } & \multirow[t]{2}{*}{ Total } \\
\hline & 6-9 a.m. & $\begin{array}{l}9 \text { a.m. - } \\
\text { midday }\end{array}$ & $\begin{array}{l}\text { Midday - } \\
3 \text { p.m. }\end{array}$ & 3-6 p.m. & 6-9 p.m. & $\begin{array}{l}9 \text { p.m. - } \\
6 \text { a.m. }\end{array}$ & \\
\hline $\begin{array}{l}\text { Contact with static } \\
\text { object }\end{array}$ & $198,25.9 \%$ & $341,19 \%$ & $366,19.2 \%$ & $567,18.8 \%$ & $580,25.4 \%$ & $186,19.3 \%$ & $2238,20.9 \%$ \\
\hline Cutting and tearing & $89,11.6 \%$ & $219,12.2 \%$ & $217,11.4 \%$ & $397,13.2 \%$ & $338,14.8 \%$ & $99,10.3 \%$ & $1359,12.7 \%$ \\
\hline Other fall & $73,9.5 \%$ & $181,10.1 \%$ & $190,10.0 \%$ & $362,12.0 \%$ & $189,8.3 \%$ & $90,9.3 \%$ & $108510.1 \%$ \\
\hline $\begin{array}{l}\text { Fall by stumbling, } \\
\text { tripping on same } \\
\text { level }\end{array}$ & $68,8.9 \%$ & $187,10.45$ & $163,8.6 \%$ & $297,9.8 \%$ & $258,11.3 \%$ & $83,8.6 \%$ & $1056,9.8 \%$ \\
\hline $\begin{array}{l}\text { Fall/jump from } \\
\text { lesser height (less } \\
\text { than } 1 \mathrm{~m} \text { ) }\end{array}$ & $79,10.3 \%$ & $156,8.7 \%$ & $154,8.1 \%$ & $278,9.2 \%$ & $199,8.7 \%$ & $114,11.8 \%$ & $980,9.1 \%$ \\
\hline $\begin{array}{l}\text { Contact with } \\
\text { moving object }\end{array}$ & $54,7.1 \%$ & $185,10.3 \%$ & $193,10.1 \%$ & $289,9.6 \%$ & $170,7.4 \%$ & $65,6.7 \%$ & $956,8.9 \%$ \\
\hline $\begin{array}{l}\text { Contact with } \\
\text { person }\end{array}$ & $41,5.4 \%$ & $174,9.7 \%$ & $219,11.5 \%$ & $225,7.5 \%$ & $140,6.1 \%$ & $96,10 \%$ & $895,8.3 \%$ \\
\hline $\begin{array}{l}\text { Miscellaneous } \\
\text { and unspecified } \\
\text { mechanism of } \\
\text { injury }\end{array}$ & $42,5.5 \%$ & $70,3.9 \%$ & $104,5.5 \%$ & $99,3.3 \%$ & $87,3.8 \%$ & $67,7 \%$ & $469,4.4$ \\
\hline $\begin{array}{l}\text { Bite, sting by } \\
\text { animal, human, } \\
\text { insect }\end{array}$ & $30,3.9 \%$ & $40,2.2 \%$ & $67,3.5 \%$ & $128,4.2 \%$ & $78,3.4 \%$ & $37,3.8 \%$ & $380,3.5 \%$ \\
\hline $\begin{array}{l}\text { Fall on or from } \\
\text { stairs }\end{array}$ & $21,2.7 \%$ & $57,3.2$ & $45,2.4 \%$ & $91,3 \%$ & $60,2.6 \%$ & $18,1.9 \%$ & $292,2.7 \%$ \\
\hline $\begin{array}{l}\text { Other, unspecified } \\
\text { contact }\end{array}$ & $9,1.2 \%$ & $39,2.2 \%$ & $52,2.75$ & $73,2.4 \%$ & $34,1.5 \%$ & $24,2.5 \%$ & $231,2.2 \%$ \\
\hline Burns & $26,3.4 \%$ & $36,2 \%$ & $27,1.4 \%$ & $32,1.1 \%$ & $56,2.5 \%$ & $24,2.5 \%$ & $201,1.9 \%$ \\
\hline $\begin{array}{l}\text { Fall/jump from } \\
\text { greater height } \\
\text { (more than } 1 \mathrm{~m} \text { ) }\end{array}$ & $12,1.6 \%$ & $43,2.4 \%$ & $32,1.7 \%$ & $65,2.2 \%$ & $27,1.2 \%$ & $14,1.5 \%$ & $193,1.8 \%$ \\
\hline $\begin{array}{l}\text { Contact with } \\
\text { animal }\end{array}$ & $11,1.4 \%$ & $28,1.6 \%$ & $27,1.4 \%$ & $59,2 \%$ & $27,1.2 \%$ & $28,2.9 \%$ & $180,1.7 \%$ \\
\hline $\begin{array}{l}\text { All pinch, crush, } \\
\text { puncture, pierce }\end{array}$ & $11,1.4 \%$ & $30,1.7 \%$ & $38,2 \%$ & $42,1.4 \%$ & $27,1.2 \%$ & $14,1.5 \%$ & $162,1.5 \%$ \\
\hline $\begin{array}{l}\text { Contact, splash, } \\
\text { spill }\end{array}$ & $1,0.1 \%$ & $8,0.4 \%$ & $8,0.4 \%$ & $12,0.4 \%$ & $13,0.6 \%$ & $4,0.4 \%$ & $46,0.4 \%$ \\
\hline Total & $765,100 \%$ & $1794,100 \%$ & $1902,100 \%$ & $3016,100 \%$ & $2283,100 \%$ & $963,100 \%$ & $\begin{array}{c}10,723 \\
100 \%\end{array}$ \\
\hline
\end{tabular}

tooth), compared to the other temporal periods, as shown in the Table XXIX.

Body region injury by injury time

There was a significant relationship between body region or injury and time of injury $\left(\chi^{2}=\right.$ 43.48, $\mathrm{d} f=15, p<0.001)$. The face was coded as being the predominant body region of injury for each coded time of injury, except for 6-9 a.m., when more head injuries compared to face injuries occurred, as shown in Table XXX.

Mode of separation by injury time

There was an association between mode of separation and time of injury $\left(\chi^{2}=60.04, \mathrm{~d} f=10, p<\right.$
0.001). As shown in the Table XXXI, the vast majority of cases were ED service event completed-discharged. However, discharge was less likely in the 6-9 a.m. timeslot, with admission more likely.

Type of place by injury time

The association between type of place where children were injured and time of injury was significant $\left(\chi^{2}=1399.90, \mathrm{~d} f=65, p<0.001\right)$. Additionally, it was found that the vast of majority of injuries occurred in the home, although the proportion occurring at home decreased during school hours (9 a.m. -12 noon, and 12 noon -3 p.m.), while the proportion of injuries occurring at primary or secondary school increased during these times, as shown in Table XXXII. 
Table XXIX. Nature of injury by time

\begin{tabular}{|c|c|c|c|c|c|c|c|}
\hline \multirow{2}{*}{$\begin{array}{l}\text { Nature of injury } \\
p<0.001\end{array}$} & \multicolumn{6}{|c|}{ Time of injury } & \multirow[t]{2}{*}{ Total } \\
\hline & 6-9 a.m. & $\begin{array}{l}9 \text { a.m. - } \\
\text { midday }\end{array}$ & $\begin{array}{l}\text { Midday - } \\
3 \text { p.m. }\end{array}$ & 3-6 p.m. & 6-9 p.m. & $\begin{array}{l}9 \text { p.m. - } \\
6 \text { a.m. }\end{array}$ & \\
\hline $\begin{array}{l}\text { Open wound } \\
\text { (excludes eye) }\end{array}$ & $313,40.9 \%$ & $727,40.5 \%$ & $725,38.1 \%$ & $1333,44.2 \%$ & $1063,46.6 \%$ & $366,38 \%$ & $4527,42.2 \%$ \\
\hline $\begin{array}{l}\text { Superficial (incl. } \\
\text { bruise; excl. eye) }\end{array}$ & $227,29.7 \%$ & $575,32.1 \%$ & $609,32 \%$ & $926,30.7 \%$ & $656,28.7 \%$ & $310,32.2 \%$ & $3303,30.8 \%$ \\
\hline $\begin{array}{l}\text { Eye injury (excl. } \\
\text { foreign body in } \\
\text { external eye [14.1]; } \\
\text { includes burn) }\end{array}$ & $136,17.8 \%$ & $306,17.1 \%$ & $363,19.1 \%$ & $455,15.1 \%$ & $352,15.4 \%$ & $178,18.5 \%$ & $1790,16.7 \%$ \\
\hline $\begin{array}{l}\text { Miscellaneous or } \\
\text { unspecified }\end{array}$ & $17,2.2 \%$ & $47,2.6 \%$ & $63,3.3 \%$ & $88,2.9 \%$ & $63,2.8 \%$ & $35,3.6 \%$ & $313,2.9 \%$ \\
\hline $\begin{array}{l}\text { Fracture and } \\
\text { dislocation (excludes } \\
\text { tooth) }\end{array}$ & $14,1.8 \%$ & $47,2.6 \%$ & $55,2.9 \%$ & $67,2.2 \%$ & $37,1.6 \%$ & $17,1.8 \%$ & $237,2.2 \%$ \\
\hline $\begin{array}{l}\text { Dental injury (incl. } \\
\text { fractured tooth) }\end{array}$ & $14,1.8 \%$ & $34,1.9 \%$ & $35,1.8 \%$ & $81,2.7 \%$ & $46,2 \%$ & $17,1.8 \%$ & $227,2.1 \%$ \\
\hline $\begin{array}{l}\text { Burn or corrosion } \\
\text { (excl. eye) }\end{array}$ & $25,3.3 \%$ & $30,1.7 \%$ & $23,1.2 \%$ & $29,1 \%$ & $48,2.1 \%$ & $21,2.2 \%$ & $176,1.6 \%$ \\
\hline $\begin{array}{l}\text { Intracranial } \\
\text { injury (includes } \\
\text { concussion) }\end{array}$ & $13,1.7 \%$ & $23,1.3 \%$ & $19,1 \%$ & $19,0.6 \%$ & $11,0.5 \%$ & $9,0.9 \%$ & $94,0.9 \%$ \\
\hline $\begin{array}{l}\text { Effect of venom; any } \\
\text { insect bite }\end{array}$ & $6,0.8 \%$ & $5,0.3 \%$ & $10,0.5 \%$ & $18,0.6 \%$ & $7,0.3 \%$ & $10,1 \%$ & $56,0.5 \%$ \\
\hline Total & $765,100 \%$ & $1794,100 \%$ & $1902,100 \%$ & $3016,100 \%$ & $\begin{array}{c}2283,963 \\
100 \%\end{array}$ & $963,100 \%$ & $\begin{array}{c}10,723 \\
100 \%\end{array}$ \\
\hline
\end{tabular}

Table XXX. Body region injury by time

\begin{tabular}{|c|c|c|c|c|c|c|c|}
\hline \multirow{2}{*}{$\begin{array}{l}\text { Body region } \\
p<0.001\end{array}$} & \multicolumn{6}{|c|}{ Time of injury } & \multirow[t]{2}{*}{ Total } \\
\hline & 6-9 a.m. & $\begin{array}{l}9 \text { a.m. - } \\
\text { midday }\end{array}$ & $\begin{array}{l}\text { Midday - } \\
3 \text { p.m. }\end{array}$ & 3-6 p.m. & 8-9 p.m. & $\begin{array}{l}9 \text { p.m. - } \\
6 \text { a.m. }\end{array}$ & \\
\hline Face (excludes eye) & $277,36.2 \%$ & $728,40.6 \%$ & $776,40.8 \%$ & $1254,41.6 \%$ & $954,41.8 \%$ & $402,41.7 \%$ & $4391,40.9 \%$ \\
\hline Head (excludes face) & $320,41.8 \%$ & $689,38.4 \%$ & $688,36.2 \%$ & $1169,38.8 \%$ & $890,39 \%$ & $326,33.9 \%$ & $4082,38.1 \%$ \\
\hline $\begin{array}{l}\text { Body location NOT } \\
\text { REQUIRED }\end{array}$ & $153,20 \%$ & $349,19.5 \%$ & $418,22 \%$ & $535,17.75$ & $413,18.1 \%$ & $214,22.2 \%$ & $2082,19.4 \%$ \\
\hline $\begin{array}{l}\text { Multiple injuries } \\
\text { (involving more than } \\
\text { one bodily location) }\end{array}$ & $15,2 \%$ & $28,1.6 \%$ & $20,1.1 \%$ & $58,1.9 \%$ & $26,1.1 \%$ & $21,2.2 \%$ & $168,1.6 \%$ \\
\hline Total & $765,100.0 \%$ & $\begin{array}{l}1794 \\
100.0 \%\end{array}$ & $\begin{array}{l}1902 \\
100.0 \%\end{array}$ & $\begin{array}{l}3016 \\
100.0 \%\end{array}$ & $\begin{array}{c}2283 \\
100.0 \%\end{array}$ & $963,100.0 \%$ & $\begin{array}{l}10,723 \\
100.0 \%\end{array}$ \\
\hline
\end{tabular}

Table XXXI. Mode of separation by injury time

\begin{tabular}{|c|c|c|c|c|c|c|c|}
\hline \multirow{2}{*}{$\begin{array}{l}\text { Mode of separation } \\
p<0.001\end{array}$} & \multicolumn{6}{|c|}{ Time of injury } & \multirow[t]{2}{*}{ Total } \\
\hline & 6-9 a.m. & $\begin{array}{l}9 \text { a.m. - } \\
\text { midday }\end{array}$ & $\begin{array}{l}\text { Midday - } \\
3 \text { p.m. }\end{array}$ & 3-6 p.m. & 8-9 p.m. & $\begin{array}{l}9 \text { p.m. - } \\
6 \text { a.m. }\end{array}$ & \\
\hline $\begin{array}{l}\text { ED service event } \\
\text { completed - } \\
\text { discharged }\end{array}$ & $605,79.1 \%$ & $1470,81.9 \%$ & $1571,82.6 \%$ & $2508,83.2 \%$ & $1936,84.8 \%$ & $814,84.5 \%$ & $8904,83 \%$ \\
\hline $\begin{array}{l}\text { Admitted to same or } \\
\text { other hospital }\end{array}$ & $152,19.9 \%$ & $302,16.8 \%$ & $317,16.7 \%$ & $472,15.6 \%$ & $291,12.7 \%$ & $127,13.2 \%$ & $1661,15.5 \%$ \\
\hline $\begin{array}{l}\text { Did not wait/left } \\
\text { after treatment } \\
\text { commenced }\end{array}$ & $8,1.0 \%$ & $22,1.2 \%$ & $14,0.7 \%$ & $36,1.2 \%$ & $56,2.5 \%$ & $22,2.3 \%$ & $158,1.5 \%$ \\
\hline Total & $765,100 \%$ & $1794,100 \%$ & $1902,100 \%$ & $3016,100 \%$ & $\begin{array}{c}2283,963 \\
100 \%\end{array}$ & $963,100 \%$ & $\begin{array}{c}10,723 \\
100 \%\end{array}$ \\
\hline
\end{tabular}


Gender by injury time

The relationship between gender and time of injury was significant $\left(\chi^{2}=16.022, \mathrm{~d} f=5, p=0.007\right)$. Males were proportionally more likely to present to $E D$ at 6-9 a.m., whereas females were proportionally more likely to present in the evening (6-9 p.m.), or overnight (9 p.m. -6 a.m.), as shown in the Table XXXIII.

Age group by injury time

There was a significant association between age group and time of injury $\left(\chi^{2}=229.157, \mathrm{~d} f=20, p<\right.$ 0.001. Infants were overrepresented at 6-9 a.m., infants and 1-2-year-olds were overrepresented at 6-9 a.m., and 5-9-year-olds were overrepresented at midday -3 p.m. and 3-6 p.m., as shown in the Table XXXIV.

Day of week of injury by time

According to the day of the week with the time, the association of the day of the week and the time of injury was significant $\left(\chi^{2}=95.027\right.$, $\mathrm{d} f=30, p<0.001)$. Additionally, it was found that the majority of injuries occurred on a Sunday and for all the coded temporal periods. However, the distribution of injury according to temporal hours of days were fairly heterogeneous within the days, with a peak found at 3-6 m on Sundays. However, more cases of injuries occurred on Fridays for all the coded temporal periods, as shown in the Table XXXV.

\section{Season of injury by injury time}

The association between season and time of injury was significant $\left(\chi^{2}=83.359, \mathrm{df}=15, p<\right.$ 0.001). Furthermore, the injury incidence peaked in autumn (March-May) at all the coded temporal hours. However, the distribution of injuries was fairly heterogeneous in months of seasons with a peak found at 3-6 p.m. in months of autumn, as

Table XXXII. Type of place by time of injury

\begin{tabular}{|c|c|c|c|c|c|c|c|}
\hline \multirow{2}{*}{$\begin{array}{l}\text { Type of place } \\
p<0.001\end{array}$} & \multicolumn{6}{|c|}{ Time of injury } & \multirow[t]{2}{*}{ Total } \\
\hline & 6-9 a.m. & $\begin{array}{l}9 \text { a.m. - } \\
\text { midday }\end{array}$ & $\begin{array}{l}\text { Midday - } \\
3 \text { p.m. }\end{array}$ & 3-6 p.m. & 8-9 p.m. & $\begin{array}{l}9 \text { p.m. - } \\
6 \text { a.m. }\end{array}$ & \\
\hline $\begin{array}{l}\text { Home (includes } \\
\text { farmhouse) }\end{array}$ & $594,77.6 \%$ & $919,51.2 \%$ & $927,48.7 \%$ & $1968,65.3 \%$ & $1881,82.4 \%$ & $697,72.4 \%$ & $6986,65.1 \%$ \\
\hline Unspecified place & $34,4.4 \%$ & $109,6.1 \%$ & $146,7.7 \%$ & $246,8.2 \%$ & $185,8.1 \%$ & $114,11.8 \%$ & $834,7.8 \%$ \\
\hline $\begin{array}{l}\text { Primary, secondary } \\
\text { school }\end{array}$ & $36,4.7 \%$ & $231,12.9 \%$ & $319,16.8 \%$ & $110,3.6 \%$ & $14,0.6 \%$ & $31,3.2 \%$ & $741,6.9 \%$ \\
\hline $\begin{array}{l}\text { Childcare, preschool } \\
\text { centre }\end{array}$ & $36,4.7 \%$ & $162,9 \%$ & $96,5 \%$ & $106,3.5 \%$ & $5,0.2 \%$ & $23,2.4 \%$ & $428,4 \%$ \\
\hline Public park & $11,1.4 \%$ & $71,4 \%$ & $91,4.8 \%$ & $152,5 \%$ & $29,1.3 \%$ & $18,1.9 \%$ & $372,3.5 \%$ \\
\hline Oval, fields, pitch & $8,1 \%$ & $82,4.6 \%$ & $61,3.2 \%$ & $75,2.5 \%$ & $35,1.5 \%$ & $16,1.7 \%$ & $277,2.6 \%$ \\
\hline $\begin{array}{l}\text { Street or highway } \\
\text { (public road) }\end{array}$ & $21,2.7 \%$ & $34,1.9 \%$ & $38,2 \%$ & $97,3.2 \%$ & $33,1.4 \%$ & $123,1.3 \%$ & $236,2.2 \%$ \\
\hline $\begin{array}{l}\text { Miscellaneous } \\
\text { specified place }\end{array}$ & $13,1.7 \%$ & $58,3.2 \%$ & $58,3 \%$ & $70,2.3 \%$ & $13,6 \%$ & $17,1.8 \%$ & $229,2.1 \%$ \\
\hline $\begin{array}{l}\text { Recreation area } \\
\text { (informal), specified, } \\
\text { incl. amusement } \\
\text { park, aquatic } \\
\text { recreation centre }\end{array}$ & $6,0.8 \%$ & $40,2.2 \%$ & $63,3.3 \%$ & $75,2.5 \%$ & $29,1.3 \%$ & $16,1.7 \%$ & $229,2.1 \%$ \\
\hline $\begin{array}{l}\text { Trade or service } \\
\text { area, incl. shopping } \\
\text { centre, restaurant }\end{array}$ & $3,0.4 \%$ & $48,1.9 \%$ & $63,3.3 \%$ & $44,1.5 \%$ & $39,1.7 \%$ & $3,0.3 \%$ & $200,1.9 \%$ \\
\hline $\begin{array}{l}\text { Other and } \\
\text { unspecified sports } \\
\text { area }\end{array}$ & $1,0.1 \%$ & $11,0.6 \%$ & $16,0.8 \%$ & $31,1 \%$ & $12,0.5 \%$ & $7,0.7 \%$ & $78,0.7 \%$ \\
\hline $\begin{array}{l}\text { Farm(excl. Farm } \\
\text { house) }\end{array}$ & $1,0.1 \%$ & $12,0.7 \%$ & $5,0.3 \%$ & $20,0.7 \%$ & $4,0.2 \%$ & $2,0.2 \%$ & $44,0.4 \%$ \\
\hline $\begin{array}{l}\text { Bush, remote or } \\
\text { undeveloped place }\end{array}$ & $1,0.1 \%$ & $10,0.6 \%$ & $10,0.5 \%$ & $16,0.5 \%$ & $2,0.1 \%$ & $3,0.3 \%$ & 42. $0.4 \%$ \\
\hline $\begin{array}{l}\text { Hospital, Medical } \\
\text { clinic or other health } \\
\text { service }\end{array}$ & $0,0 \%$ & $7,0.4 \%$ & $9,0.5 \%$ & $6,0.2 \%$ & $2,0.1 \%$ & $3,0.3 \%$ & $27,0.3 \%$ \\
\hline Total & $765,100 \%$ & $1794,100 \%$ & $1902,100 \%$ & $3016,100 \%$ & $\begin{array}{c}2283,963 \\
100 \%\end{array}$ & $963,100 \%$ & $\begin{array}{c}10,723 \\
100 \%\end{array}$ \\
\hline
\end{tabular}


Table XXXIII. Gender injury by time

\begin{tabular}{|lccccccc|}
\hline $\begin{array}{l}\text { Gender } \\
\boldsymbol{p}=\mathbf{0 . 0 0 7}\end{array}$ & $\mathbf{6 - 9}$ a.m. & $\begin{array}{c}\mathbf{9} \text { a.m. - } \\
\text { midday }\end{array}$ & $\begin{array}{c}\text { Midday - } \\
\text { 3 p.m. }\end{array}$ & 3-6 p.m. & 6-9 p.m. & \multicolumn{2}{c|}{$\begin{array}{c}\text { 9 p.m. - } \\
\text { 6 a.m. }\end{array}$} \\
\hline Male & $507,66.3 \%$ & $1165,64.9 \%$ & $1167,61.4 \%$ & $1896,62.9 \%$ & $1393,61 \%$ & $574,59.6 \%$ & $6702,62.5 \%$ \\
\hline Female & $258,33.7 \%$ & $629,35.1 \%$ & $735,38.6 \%$ & $1120,37.1 \%$ & $890,39 \%$ & $389,40.4 \%$ & $4021,37.5 \%$ \\
\hline Total & $765,100 \%$ & $1794,100 \%$ & $1902,100 \%$ & $3016,100 \%$ & 2283,963, & $963,100 \%$ & 10,723, \\
& & & & & $100 \%$ & & $100 \%$ \\
\hline
\end{tabular}

Table XXXIV. Age groups by time of injury

\begin{tabular}{|c|c|c|c|c|c|c|c|}
\hline \multirow{2}{*}{$\begin{array}{l}\text { Age group } \\
p<0.001\end{array}$} & \multicolumn{6}{|c|}{ Time of injury } & \multirow[t]{2}{*}{ Total } \\
\hline & 6-9 a.m. & $\begin{array}{l}9 \text { a.m. - } \\
\text { midday }\end{array}$ & $\begin{array}{l}\text { Midday - } \\
3 \text { p.m. }\end{array}$ & 3-6 p.m. & 6-9 p.m. & $\begin{array}{l}9 \text { p.m.- } \\
6 \text { a.m. }\end{array}$ & \\
\hline $\begin{array}{l}\text { Infant } \\
\text { (0 years) }\end{array}$ & $90,11.8 \%$ & $103,5.7 \%$ & $112,5.9 \%$ & $165,5.5 \%$ & $144,6.3 \%$ & $81,8.4 \%$ & $695,6.5 \%$ \\
\hline $1-2$ years & $317,41.4 \%$ & $664,37 \%$ & $512,26.9 \%$ & $897,29.7 \%$ & $826,36.2 \%$ & $295,30.6 \%$ & $3511,32.7 \%$ \\
\hline $3-4$ years & $142,18.6 \%$ & $342,19.1 \%$ & $366,19.2 \%$ & $640,21.2 \%$ & $513,22.5 \%$ & $198,20.6 \%$ & $2201,20.5 \%$ \\
\hline $5-9$ years & $163,21.3 \%$ & $458,25.5 \%$ & $632,33.2 \%$ & $986,32.7 \%$ & $610,26.7 \%$ & $271,28.1 \%$ & $3120,29.1 \%$ \\
\hline $\begin{array}{l}10-12 \\
\text { years }\end{array}$ & $53,6.9 \%$ & $227,12.7 \%$ & $280,14.7 \%$ & $328,10.9 \%$ & $190,8.3 \%$ & $118,12.3 \%$ & $1196,11.2 \%$ \\
\hline Total & $765,100.0 \%$ & $1794,100.0 \%$ & $1902,100.0 \%$ & $3016,100.0 \%$ & $2283,100.0 \%$ & $963,100.0 \%$ & $\begin{array}{l}10,723 \\
100.0 \%\end{array}$ \\
\hline
\end{tabular}

Table XXXV. Day of week injury by time

\begin{tabular}{|c|c|c|c|c|c|c|c|}
\hline \multirow{2}{*}{$\begin{array}{l}\text { Day of week } \\
p<0.001\end{array}$} & \multicolumn{6}{|c|}{ Time of injury } & \multirow[t]{2}{*}{ Total } \\
\hline & 6-9 a.m. & $\begin{array}{c}9 \text { a.m. - mid- } \\
\text { day }\end{array}$ & $\begin{array}{l}\text { Midday - } \\
3 \text { p.m. }\end{array}$ & 3-6 p.m. & 8-9 p.m. & 9 p.m.-6 a.m. & \\
\hline Sun & $113,14.8 \%$ & $384,21.4 \%$ & $382,20.1 \%$ & $555,18.4 \%$ & $384,15.2 \%$ & $143,14.8 \%$ & $1925,18 \%$ \\
\hline Mon & $105,13.7 \%$ & $215,12 \%$ & $221,11.6 \%$ & $\begin{array}{c}409,317 \\
13.9 \%\end{array}$ & $317,13.9 \%$ & $131,13.6 \%$ & $1398,13 \%$ \\
\hline Tue & $97,12.7 \%$ & $204,11.4 \%$ & $209,11 \%$ & $365,12.1 \%$ & $309,13.3 \%$ & $105,10.9 \%$ & $1289,12 \%$ \\
\hline Wed & $116,15.2 \%$ & $246,13.7 \%$ & $238,12.5 \%$ & $401,13.3 \%$ & $326,14.3 \%$ & $101,10.5 \%$ & $1428,13.3 \%$ \\
\hline Thu & $104,13.6 \%$ & $205,11.4 \%$ & $255,13.4 \%$ & $387,12.8 \%$ & $300,13.1 \%$ & $139,14.4 \%$ & $1390,13 \%$ \\
\hline Fri & $122,15.9 \%$ & $225,12.5 \%$ & $263,13.8 \%$ & $414,13.7 \%$ & $355,15.5 \%$ & $156,16.2 \%$ & $1535,14.3 \%$ \\
\hline Sat & $108,14.1 \%$ & $315,17.6 \%$ & $334,17.6 \%$ & $485,16.1 \%$ & $328,14.4 \%$ & $188,19.5 \%$ & $1758,16.4 \%$ \\
\hline Total & $765,100.0 \%$ & $1794,100.0 \%$ & $1902,100.0 \%$ & $3016,100.0 \%$ & $2283,100.0 \%$ & $963,100.0 \%$ & $\begin{array}{l}10,723 \\
100.0 \%\end{array}$ \\
\hline
\end{tabular}

shown in the Table XXXVI. However, this analysis was not conducted with 2016 removed.

Month of injury by time

According to month of injury, it was found that there was a strong evidence of an association between month and time of injury $\left(\chi^{2}=133.89, \mathrm{~d} f=\right.$ $55, p<0.00)$. Additionally, the majority of injuries occurred in January in the total sample. However, the distribution of injuries was fairly heterogeneous according to the time of injury over the months, with peaks in May at 3-6 p.m. and March 3-6 p.m., as shown in Table XXXVII. However, this analysis was not conducted with 2016 removed.
Age-specific analyses

As previously described, the majority of head and facial injuries occurred in children aged 1-2 years $(n=3511,32 \%)$, followed by children aged 5-9 years $(n=3120,29.1 \%)$, then $3-4$ years $(n=$ $2201,20.5 \%)$, and $10-12$ years ( $n=1196,11.2 \%)$. The fewest injuries occurred in infants less than 1 year old $(n=695,6.5 \%)$. Because so many of the injury and event characteristics varied with age, as described in section 3.2, the epidemiology of head and facial injuries will now be described in further detail by age group. 
Table XXXVI. Season by time of injury

\begin{tabular}{|c|c|c|c|c|c|c|c|}
\hline \multirow{2}{*}{$\begin{array}{l}\text { Season } \\
P<0.001\end{array}$} & \multicolumn{6}{|c|}{ Time of injury } & \multirow[t]{2}{*}{ Total } \\
\hline & 6-9 a.m. & $\begin{array}{l}9 \text { a.m. - } \\
\text { midday }\end{array}$ & $\begin{array}{l}\text { Midday - } \\
3 \text { p.m. }\end{array}$ & 3-6 p.m. & 8-9 p.m. & 9 p.m.-6 a.m. & \\
\hline $\begin{array}{l}\text { Autumn (March } \\
\text { - May) }\end{array}$ & $225,29.5 \%$ & $542,30.2 \%$ & $538,28.3 \%$ & $846,28.1 \%$ & $598,26.2 \%$ & $231,24 \%$ & $2980,27.8 \%$ \\
\hline $\begin{array}{l}\text { Summer } \\
\text { (Dec-Feb) }\end{array}$ & $175,22.9 \%$ & $377,21 \%$ & $466,24.5 \%$ & $764,25.3 \%$ & $683,29.9 \%$ & $291,30.2 \%$ & $2756,25.7 \%$ \\
\hline $\begin{array}{l}\text { Spring } \\
\text { (Sept-Nov) }\end{array}$ & $207,27.1 \%$ & $398,22.2 \%$ & $394,20.7 \%$ & $674,22.3 \%$ & $483,21.2 \%$ & $231,24 \%$ & $2387,22.3 \%$ \\
\hline $\begin{array}{l}\text { Winter } \\
\text { (June - Aug) }\end{array}$ & $158,20.7 \%$ & $477,26.6 \%$ & $504,26.5 \%$ & $732,24.3 \%$ & $519,22.7 \%$ & $210,21.8 \%$ & $2600,24.2 \%$ \\
\hline Total & $765,100.0 \%$ & $1794,100.0 \%$ & $1902,100.0 \%$ & $3016,100.0 \%$ & $2283,100.0 \%$ & $963,100.0 \%$ & $\begin{array}{l}10,723 \\
100.0 \%\end{array}$ \\
\hline
\end{tabular}

Table XXXVII. Injury month by time

\begin{tabular}{|c|c|c|c|c|c|c|c|}
\hline \multirow{2}{*}{$\begin{array}{l}\text { Month } \\
p<0.001\end{array}$} & \multicolumn{6}{|c|}{ Time of injury } & \multirow[t]{2}{*}{ Total } \\
\hline & 6-9 a.m. & $\begin{array}{l}9 \text { a.m. - } \\
\text { midday }\end{array}$ & $\begin{array}{l}\text { Midday - } \\
3 \text { p.m. }\end{array}$ & 3-6 p.m. & 8-9 p.m. & 9 p.m.-6 a.m. & \\
\hline January & $60,7.8 \%$ & $129,7.2 \%$ & $169,8.9 \%$ & $277,9.2 \%$ & $283,12.4 \%$ & $110,11.4 \%$ & $1028,9.6 \%$ \\
\hline February & $68,8.9 \%$ & $133,7.4 \%$ & $148,7.8 \%$ & $242,8.0 \%$ & $209,9.2 \%$ & $94,9.8 \%$ & $894,8.3 \%$ \\
\hline March & $70,9.2 \%$ & $177,9.9 \%$ & $168,8.8 \%$ & $282,9.4 \%$ & $213,9.3 \%$ & $89,9.2 \%$ & $999,9.3 \%$ \\
\hline April & $76,9.9 \%$ & $162,9.0 \%$ & $166,8.7 \%$ & $268,8.9 \%$ & $195,8.5 \%$ & $67,7.0 \%$ & $934,8.7 \%$ \\
\hline May & $79,10.3 \%$ & $203,11.3 \%$ & $204,10.7 \%$ & $296,9.8 \%$ & $190,8.3 \%$ & $75,7.8 \%$ & $1047,9.8 \%$ \\
\hline June & $70,9.2 \%$ & $164,9.1 \%$ & $206,10.8 \%$ & $254,8.4 \%$ & $196,8.6 \%$ & $69,7.2 \%$ & $959,8.9 \%$ \\
\hline July & $45,5.9 \%$ & $138,7.7 \%$ & $145,7.6 \%$ & $241,8.0 \%$ & $158,6.9 \%$ & $62,6.4 \%$ & $789,7.4 \%$ \\
\hline August & $43,5.6 \%$ & $175,9.8 \%$ & $153,8.0 \%$ & $237,7.9 \%$ & $165,7.2 \%$ & $79,8.2 \%$ & $852,7.9 \%$ \\
\hline September & $63,8.2 \%$ & $141,7.9 \%$ & $137,7.2 \%$ & $205,6.8 \%$ & $149,6.5 \%$ & $88,9.1 \%$ & $783,7.3 \%$ \\
\hline October & $74,9.7 \%$ & $125,7.0 \%$ & $127,6.7 \%$ & $256,8.5 \%$ & $177,7.8 \%$ & $67,7.0 \%$ & $826,7.7 \%$ \\
\hline November & $70,9.2 \%$ & $132,7.4 \%$ & $130,6.8 \%$ & $213,7.1 \%$ & $157,6.9 \%$ & $76,7.9 \%$ & $778,7.3 \%$ \\
\hline December & $47,6.1 \%$ & $115,6.4 \%$ & $149,7.8 \%$ & $245,8.1 \%$ & $191,8.4 \%$ & $87,9.0 \%$ & $834,7.8 \%$ \\
\hline Total & $765,100.0 \%$ & $\begin{array}{c}1794 \\
100.0 \%\end{array}$ & $\begin{array}{c}1902 \\
100.0 \%\end{array}$ & $\begin{array}{c}3016 \\
100.0 \%\end{array}$ & $\begin{array}{c}2283 \\
100.0 \%\end{array}$ & $963,100.0 \%$ & $\begin{array}{l}10,723 \\
100.0 \%\end{array}$ \\
\hline
\end{tabular}

Infants (0 years old)

There were a total of 695 children in this age group. Overall in this infant age group, most injuries occurred at home $(n=596,85.8 \%)$ (Table XIV), when infants were being nursed or cared for (330, 47.5\%) (Table XVI).

The first three most common mechanisms of injury within this age group were fall/jump from lesser height (less than $1 \mathrm{~m}$ ) (160 cases, 23.0\%); contact with a static object (125 cases, $18 \%)$; and other falls (115 cases, $16.5 \%$ ).

The most common ICD diagnosis for each of the three mechanisms of injury was 'superficial injury of head part not otherwise specified'. Other frequent diagnoses were 'superficial injury of other parts of head' and open would of head or of 'lip and oral cavity'.

For all of the three most common mechanisms of injury in this age group, the bedroom was the main part of the place where the infants were injured, and for the two fall-related mechanism categories, bed (excludes bunkbed and cot) was the most common injury factor. For the contact with a static object mechanism category, 'living, family, and rumpus room' was coded as being the most predominant place where injuries occurred, when the children were being nursed, resting, sleeping, eating, other, and playing, for the three most common mechanisms of injury. 'Table, desk, bench, etc.' was the main major injury factor.

The highest frequency of injuries occurred at 3-6 p.m. for the most common mechanism of injury, whereas for the second most common mechanism of injury, the greatest incidence of injury occurred at 3-6 p.m. For the third most common mechanism of injury, the highest incidence of injury occurred at 6-9 p.m. (Table XXXVIII). 
Table XXXVIII. Injury characteristics by mechanism of injury in the $0-1$ years age group $(n=695,6.5 \%)$

\begin{tabular}{|c|c|c|}
\hline $\begin{array}{l}\text { Mechanism of injury } 1 \text { : } \\
\text { fall/jump from lesser height } \\
(\text { less than } 1 \mathrm{~m}) \\
(n=160)\end{array}$ & $\begin{array}{l}\text { Mechanism of injury } 2 \text { : } \\
\text { contact with static object } \\
(n=125)\end{array}$ & $\begin{array}{l}\text { Mechanism of injury } 3: \\
\text { other falls } \\
(n=115)\end{array}$ \\
\hline $\begin{array}{l}\text { ICD: } \\
\text { - Superficial injury of head part not } \\
\text { otherwise specified }(100,62.5 \%) \\
\text { - Superficial injury of other parts of } \\
\text { head }(12,7.5 \%) \\
\text { - Open wound of lip and oral cavity } \\
(10,6.3 \%)\end{array}$ & $\begin{array}{l}\text { ICD: } \\
\text { - Superficial injury of head part not } \\
\text { otherwise specified }(37,29.6 \%) \\
\text { - Open wound of other parts of } \\
\text { head }(16,12.8 \%) \\
\text { - Superficial injury of other parts of } \\
\text { head }(12,9.6 \%)\end{array}$ & $\begin{array}{l}\text { ICD: } \\
\text { - Superficial injury of head part not } \\
\text { otherwise specified }(69,60 \%) \\
\text { - Superficial injury of other parts of } \\
\text { head }(8,7 \%) \\
\text { - Superficial injury of head, and } \\
\text { open wound of lip and oral cavity } \\
(7,6.1 \%) \text { for each }\end{array}$ \\
\hline $\begin{array}{c}\text { Gender: } \\
\text { Male: }(90,56.25 \%) \\
\text { Female: }(70,43.75 \%)\end{array}$ & $\begin{array}{c}\text { Gender: } \\
\text { Male: }(79,63.2 \%) \\
\text { Female: }(46,36.8 \%)\end{array}$ & $\begin{array}{c}\text { Gender: } \\
\text { Male: }(64,55.65 \%) \\
\text { Female: }(51,44.35 \%)\end{array}$ \\
\hline Type of place: home $(139,86.9 \%)$ & Type of place: home $(109,87.2 \%)$ & Type of place: home $(94,81.7 \%)$ \\
\hline $\begin{array}{l}\quad \text { Activity: } \\
\text { - Being nursed or care for }(78,48.8 \%) \\
\text { - Resting, sleeping, eating, other } \\
\quad(36,22.5 \%) \\
\text { - Playing }(27,16.9 \%)\end{array}$ & $\begin{array}{l}\quad \text { Activity: } \\
\text { - Being nursed or cared for }(55,44 \%) \text {, } \\
\text { - Plying }(28,22.4 \%) \\
\text { - Resting, sleeping, eating, other } \\
\quad(15,12 \%)\end{array}$ & $\begin{array}{l}\quad \text { Activity: } \\
\text { - Being nursed or cared }(73,63.5 \%) \\
\text { - Resting, sleeping, eating, other } \\
\text { (23, 20\%) } \\
\text { - Playing }(9,7.8 \%)\end{array}$ \\
\hline $\begin{array}{l}\text { Major injury factor: } \\
\text { - Bed (excludes bunk bed and cot) } \\
\text { (61, 38.1\%) } \\
\text { - Sofa, couch, lounge, divan, etc. } \\
\text { (21, } 13.1 \%) \\
\text { - Table, desk, bench, etc. }(11,6.9 \%)\end{array}$ & $\begin{array}{l}\text { Major injury factor: } \\
\text { - Table, desk, bench, etc. }(27,21.6 \%) \\
\text { - Bed (excludes bunk bed and cot) } \\
\text { (14, } 11.2 \%) \\
\text { - Table and cabinet, rack, room } \\
\text { divider, shelf }(11,8.8 \%) \text { for each }\end{array}$ & $\begin{array}{l}\quad \text { Major injury factor: } \\
\text { - Bed (excludes bunk bed and cot) } \\
\text { (27, } 23.5 \%) \\
\text { - Baby pram, pusher etc. }(13,11.3 \%) \\
\text { - Floor }(10,8.7 \%)\end{array}$ \\
\hline $\begin{array}{l}\quad \text { Part of place: } \\
\text { - Bed room (62, 38.8\%) } \\
\text { - Unspecified part of place }(45, \\
28.1 \%) \\
\text { - Other, interior }(14,8.8 \%)\end{array}$ & $\begin{array}{l}\text { Part of place: } \\
\text { - Unspecified part of place }(31,24.8 \%) \\
\text { - Other, interior }(30,24 \%) \\
\text { - Living, family, rumpus room and } \\
\text { bedroom with }(23,18.4 \%) \text { for each }\end{array}$ & $\begin{array}{r}\text { Part of place: } \\
\text { - Bedroom }(94,81.7 \%)\end{array}$ \\
\hline $\begin{array}{c}\text { Time: } \\
\text { 3-6 p.m. }(42,26.3 \%) \\
p=0.020\end{array}$ & $\begin{array}{c}\text { Time: } \\
\text { 3-6 p.m. }(32,25.6 \%) \\
p=0.007\end{array}$ & $\begin{array}{c}\text { Time: } \\
\text { 6-9 p.m. }(26,22.6 \%) \\
p=0.210\end{array}$ \\
\hline
\end{tabular}

In total, $55.54 \%$ of injuries in this age group were caused by falls $(n=386)$.

\section{1-2-years age group}

Overall in this 1-2-years age group, most injuries occurred at home ( $n=2714,38.8 \%)$ (Table XIV), when children were engaged in playing (1423, 37.4\%) (Table XVI).

The first three most common mechanisms of injury within this age group were contact with static object (857, 24.4\%), cutting and tearing (539, 15.4\%), and falling/stumbling/tripping on same level $(521,14.8 \%)$. There were a total of 3511 children in this age group within the dataset.

Open wound of other parts of head, open wound of the lip and oral cavity were the most common ICDs for the first and second mechanisms of injury. However, open wound of the lip and oral cavity was the most common mechanism of injury with the third most common mechanism with this age group.

For all of the three most common mechanisms of injury in this age group, unspecified part of place was the main part of place where the children were injured for the three most common mechanisms of injury. Table, desk. bench, etc. was the most common injury factor (Table XXXIX) for the most common mechanism of injury, whereas other unspecified factor was the most common injury factor for the second and third most common mechanisms of injury.

The highest frequency of injuries occurred at 6-9 p.m. for the most common mechanism of injury, whereas for the second most common mechanism of injury the greatest incidence of injury occurred at 3-6 p.m. For the third most common mechanism of injury, the highest incidence of injury occurred at 6-9 p.m. (Table XXXIX).

\section{3-4-years age group}

Overall in this 3-4-years age group, most injuries occurred at home ( $n=1524,21.8 \%$ ) (Table XIV), when children were playing (1006, 26.4\%) (Table XVI).

The first three most common mechanisms of injury within this age group were contact with 
Table XXXIX. Injury characteristics by mechanism of injury, with age group $1-2$ years $(n=3511,32.7 \%)$

\begin{tabular}{|c|c|c|}
\hline $\begin{array}{l}\text { Mechanism of injury } 1 \text { : } \\
\text { contact with a static object } \\
(n=857)\end{array}$ & $\begin{array}{l}\text { Mechanism of injury } 2 \text { : } \\
\text { cutting tearing } \\
(n=539)\end{array}$ & $\begin{array}{c}\text { Mechanism of injury 3: } \\
\text { falling/stumbling/tripping on same } \\
\text { level } \\
(n=521)\end{array}$ \\
\hline $\begin{array}{l}\text { ICD: } \\
\text { - Open wound of other parts of } \\
\text { head }(211,24.6 \%) \\
\text { - Open wound of lip and oral cavity } \\
(113,13.2 \%) \\
\text { - Superficial injury of head part no } \\
\text { otherwise specified }(108,12.6 \%)\end{array}$ & $\begin{array}{l}\text { ICD: } \\
\text { - Open wound of other parts of } \\
\text { head }(176,32.7 \%) \\
\text { - Open wound of lip and oral cavity } \\
\text { (167, 31\%) } \\
\text { - Open wound of eyelid and } \\
\text { periocular area }(62,31 \%)\end{array}$ & $\begin{array}{l}\text { ICD: } \\
\text { - Open wound of lip and oral cavity } \\
(97,18.6 \%) \\
\text { - Open wound of other parts of } \\
\text { head }(95,18.2 \%) \\
\text { - Superficial injury of head parts of } \\
\text { no otherwise specified }(94,18 \%)\end{array}$ \\
\hline $\begin{array}{c}\text { Gender: } \\
\text { Male: } 540,63.01 \% \\
\text { Female: } 317,36.99 \% \\
\end{array}$ & $\begin{array}{c}\text { Gender: } \\
\text { Male: } 337,62.52 \% \\
\text { Female: } 202,37.48 \% \\
\end{array}$ & $\begin{array}{c}\text { Gender: } \\
\text { Male: } 322,61.80 \% \\
\text { Female: } 199,38.20 \% \\
\end{array}$ \\
\hline Type of pla & Type of place & Type of plac \\
\hline $\begin{array}{l}\text { Activity: } \\
\text { - Playing (428, 49.9\%) } \\
\text { - Resting, sleeping, eating, and } \\
\text { other (138, 16.1\%) } \\
\text { - Unspecified activity }(92,10.7 \%)\end{array}$ & $\begin{array}{l}\text { Activity: } \\
\text { - Playing (214, 39.7\%) } \\
\text { - Resting, sleeping, eating, and } \\
\text { other (107, 19.9\%) } \\
\text { - Unspecified activity (102, 18.9\%) }\end{array}$ & $\begin{array}{l}\text { Activity: } \\
\text { - Playing (181, 34.7\%) } \\
\text { - Resting, sleeping, eating, and } \\
\text { other (140, } 26.9 \%) \\
\text { - Being nursed or care for }(105,20.2 \%)\end{array}$ \\
\hline $\begin{array}{l}\quad \text { Major injury factor: } \\
\text { - Table, desk. Bench. Etc. }(245 \text {, } \\
\text { 28.6\%) } \\
\text { - Other or unspecified factor ( } 30 \text {, } \\
3.5 \%) \\
\text { - Floor }(29,3.4 \%)\end{array}$ & $\begin{array}{l}\quad \text { Major injury factor: } \\
\text { - Other unspecified factor }(145, \\
\text { 26.9\%) } \\
\text { - Table, desk, bench, etc. }(61,11.3 \%) \\
\text { - Other toy }(28,5.2 \%)\end{array}$ & $\begin{array}{l}\quad \text { Major injury factor: } \\
\text { - Other or unspecified factor (120, } \\
\text { 23\%) } \\
\text { - Floor }(106,20.3 \%) \\
\text { - Bathtub, shower }(61,11.7 \%)\end{array}$ \\
\hline $\begin{array}{l}\text { Part of place: } \\
\text { - Unspecified part of place (239, } \\
27.9 \%) \\
\text { - Living, family, rumpus room (142, } \\
\text { 16.6\%) } \\
\text { - Bedroom }(126,14.7 \%)\end{array}$ & $\begin{array}{l}\text { Part of place: } \\
\text { - Unspecified part of place ( } 286, \\
53.1 \%) \\
\text { - Living, family, rumpus room (52, } \\
\text { 9.6\%) } \\
\text { - Bedroom }(40,7.4 \%)\end{array}$ & $\begin{array}{l}\quad \text { Part of place: } \\
\text { - Unspecified part of place }(203,39 \%) \\
\text { - Bathroom ( } 97,18.6 \%) \\
\text { - Living, family, rumpus room (64, } \\
\text { 8.8\%) }\end{array}$ \\
\hline $\begin{array}{c}\text { Time: } 6-9 \text { p.m. }(221,25.8 \%) \\
p<0.001\end{array}$ & $\begin{array}{c}\text { Time: } 3-6 \text { p.m. }(146,27.1 \%) \\
p=0.090\end{array}$ & $\begin{array}{c}\text { Time: } 6-9 \text { p.m. }(136,26.1 \%) \\
p<0.001\end{array}$ \\
\hline
\end{tabular}

static object (542, 25.6\%), cutting and tearing (337, 15.3\%), and falling/stumbling/tripping on the same level $(225,16.2 \%)$. There were a total of 2201 children in this age group within the dataset.

Open wound of other parts of head was the most frequent ICD for the three most common mechanisms of injury within this age group, followed by open wound of other parts of head, and superficial injury of head part not otherwise specified, for the most common mechanism of injury. However, open wound of the lip and oral cavity was the second ICD for both the second and third most common mechanisms of injury, followed by open wound of the eyelid and periocular area for the second mechanism of injury, and superficial injury of head part not otherwise specified for the third common mechanism of injury.

For all of the three most common mechanisms of injury, unspecified part of place was the main part of place where the children were injured. Table, desk, bench, etc. was the most common injury factor for the first and second most common mechanisms of injury, whereas other or unspeci- fied factor was the main major factor for the third mechanism of injury category.

The highest frequency of injuries occurred at 6-9 p.m. for the most common mechanism of injury, whereas for the second most common mechanism of injury, the greatest incidence of injury occurred at 3-6 p.m. For the third most common mechanism of injury, the highest incidence of injury occurred at 3-6 p.m. (Table XL).

\section{5-9-years age group}

Overall in this age group, most injuries occurred at home ( $n=1709,24.5 \%)$ (Table XIV), when children were engaging in playing (1020, 26.8\%) (Table XVI).

The first three most common mechanisms of injury within this age group were contact with static object $(585,18.8 \%)$, contact with moving object $(399,12.8 \%)$, and contact with person (388, 12.4\%). There were a total of 3120 children in this age group within the dataset.

Open wound of other parts of head was observed to be the most common ICD for the most common of injury mechanism category, followed 
Table XL. Injury characteristics by mechanism of injury for the 3-4-years age group $(n=2201,20.5 \%)$

\begin{tabular}{|c|c|c|}
\hline $\begin{array}{l}\text { Mechanism of injury } 1 \text { : } \\
\text { contact with static object } \\
\qquad(n=542)\end{array}$ & $\begin{array}{l}\text { Mechanism of injury } 2 \text { : } \\
\text { cutting and tearing } \\
\qquad(n=337)\end{array}$ & $\begin{array}{l}\text { Mechanism of injury } 3 \text { : } \\
\text { falling/stumbling/tripping on the } \\
\text { same level } \\
(n=225)\end{array}$ \\
\hline $\begin{array}{l}\text { ICD: } \\
\text { - Open wound of other parts of } \\
\text { head }(162,29.9 \%) \\
\text { - Open wound of other parts of } \\
\text { head }(71,13.1 \%) \\
\text { - Superficial injury of head part no } \\
\text { otherwise specified }(63,11.6 \%)\end{array}$ & $\begin{array}{l}\text { ICD: } \\
\text { - Open wound of other parts of } \\
\text { head }(133,39.5 \%) \\
\text { - Open wound of lip and oral cavity } \\
(84,24.9 \%) \\
\text { - Open wound of eyelid and } \\
\text { periocular area }(33,9.8 \%)\end{array}$ & $\begin{array}{l}\text { ICD: } \\
\text { - Open wound of other parts of } \\
\text { head }(51,22.7 \%) \\
\text { - Open wound of lip and oral cavity } \\
\text { (50, } 22.2 \%) \\
\text {-Superficial injury of head part no } \\
\text { otherwise specified }(21,9.3 \%)\end{array}$ \\
\hline $\begin{array}{c}\text { Gender: } \\
\text { Male: } 337,62.18 \% \\
\text { Female: } 205,37.82 \%\end{array}$ & $\begin{array}{c}\text { Gender: } \\
\text { Male: } 215,63.80 \% \\
\text { Female: } 122,36.20 \%\end{array}$ & $\begin{array}{c}\text { Gender: } \\
\text { Male: } 141,62.67 \% \\
\text { Female: } 84,37.33 \%\end{array}$ \\
\hline$(418,77.1 \%)$ & $\begin{array}{l}\text { Type of place: } \\
\text { - Unspecified place }(22,6.5 \%) \\
\text { - Public park }(14,4.2 \%) \\
\text { - Trade or service area, incl. } \\
\text { shopping centre, restaurant }(12, \\
3.6 \%)\end{array}$ & $\begin{array}{l}\text { Type of place: } \\
\text { - Recreation area (informal), } \\
\text { specified, incl. amusement park, } \\
\text { aquatic recreation canter }(8,3.6 \%) \\
\text { - Street or highway (public road) }(7, \\
\text { 3.1\%) } \\
\text { - Public park }(5,2.2 \%)\end{array}$ \\
\hline $\begin{array}{l}\text { Activity: } \\
\text { - Playing (305, 56.3\%) } \\
\text { - Resting, sleeping, eating, other } \\
\text { (76, 14\%) } \\
\text { - Unspecified activity }(53,9.8 \%)\end{array}$ & $\begin{array}{l}\text { Activity: } \\
\text { - Playing (139, 41.2\%) } \\
\text { - Resting, sleeping, eating, other } \\
\text { (64, 19\%) } \\
\text { - Unspecified activity }(53,15.7 \%)\end{array}$ & $\begin{array}{l}\quad \text { Activity: } \\
\text { - Playing (95, 42.2\%) } \\
\text { - Resting, sleeping, eating, other } \\
\text { (58, 25.8\%) } \\
\text { - Unspecified activity }(29,12.9 \%)\end{array}$ \\
\hline $\begin{array}{l}\quad \text { Major injury factor: } \\
\text { - Table, desk, bench, etc. }(129, \\
23.8 \%) \\
\text { - Wall }(41,7.6 \%) \\
\text { - Other or unspecified structure and } \\
\text { fixture }(23,4.2 \%)\end{array}$ & $\begin{array}{l}\text { Major injury factor: } \\
\text { - Table, desk, bench, etc. }(44,13.1 \%) \\
\text { - Other playground equipment (15, } \\
4.5 \%) \\
\text { - Sofa, couch, lounge, divan, etc. } \\
\quad(12,3.6 \%)\end{array}$ & $\begin{array}{l}\quad \text { Major injury factor: } \\
\text { - Other or unspecified factor }(48, \\
21.3 \%) \\
\text { - Floor }(43,19.1 \%) \\
\text { - Bathtub, shower }(21,9.3 \%)\end{array}$ \\
\hline $\begin{array}{l}\quad \text { Part of place: } \\
\text { - Unspecified part of place (154, } \\
\text { 28.4\%) } \\
\text { - Living, family, rumpus room (71, } \\
\text { 13.1\%) } \\
\text { - Other, interior }(68,12.5 \%)\end{array}$ & $\begin{array}{l}\text { Part of place: } \\
\text { - Unspecified part of place (162, } \\
\text { 48.1\%) } \\
\text { - Living, family, rumpus room (31, } \\
\text { 9.2\%) } \\
\text { - Bedroom }(27,8 \%)\end{array}$ & $\begin{array}{l}\text { Part of place: } \\
\text { - Unspecified part of place }(78, \\
\text { 34.7\%) } \\
\text { - Bathroom (40, 17.8\%) } \\
\text { - Garden, park, national park, } \\
\text { backyard (17, } 7.6 \%)\end{array}$ \\
\hline $\begin{array}{c}\text { Time: } 6-9 \text { p.m. }(166,30.6 \%) \\
p<0.001\end{array}$ & $\begin{array}{c}\text { Time: } 3-6 \text { p.m. }(105,31.2 \%) \\
p<0.001\end{array}$ & 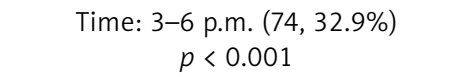 \\
\hline
\end{tabular}

by superficial injury of head part not otherwise specified and superficial injury of other part of the head. However, injury conjunctive corneal abrasion without $\mathrm{fb}$ was the most common ICD for the second and third most common mechanisms of injury within this age group, followed by open wound of other parts of head and contusion of the eyelid and periocular area for the second most common mechanism of injury, and contusion of the eyelid and periocular and open wound of other parts of the head area for the third most common mechanism of injury.

Unspecified part of place was the main part of place where children were injured for the first and third mechanisms injury category, whereas, garden, park, national park, and backyard were coded as being the main part of place for the second most common mechanism of injury category. Table, desk, bench, etc. was the most common injury factor for the most common mechanism of injury category, while ball was the most common injury factor for the second common mechanism of injury category, and person was the most common injury factor for the third most common mechanism of injury category.

The highest frequency of injuries occurred at 3-6 p.m. for the most common mechanism of injury, whereas for the second most common mechanism of injury, the greatest incidences of injury occurred at 3-6 p.m. For the third most common mechanism of injury, the highest incidences of injury occurred at 3-6 p.m. (Table XLI).

\section{0-12-years age group}

Overall in this age group, most injuries occurred at home $(n=443,6.3 \%)$ (Table XIV) when children were playing $(244,6.4 \%)$ (Table XVI). 
Table XLI. Injury characteristics by mechanism of injury in the 5-9-years age group ( $n=3120,29.1 \%)$

\begin{tabular}{|c|c|c|}
\hline $\begin{array}{l}\text { Mechanism of injury } 1: \\
\text { Contact with static object } \\
(n=585)\end{array}$ & $\begin{array}{l}\text { Mechanism of injury } 2 \text { : } \\
\text { Contact with moving object } \\
\qquad(n=399)\end{array}$ & $\begin{array}{l}\text { Mechanism of injury } 3 \text { : } \\
\text { Contact with person } \\
\quad(n=388)\end{array}$ \\
\hline $\begin{array}{l}\text { ICD: } \\
\text { - Open wound of other parts of } \\
\text { head }(136,23.2 \%) \\
\text { - Superficial injury of head part no } \\
\text { otherwise specified }(58,9.9 \%) \\
\text { - Superficial injury of other part of } \\
\text { head }(58,9.9 \%)\end{array}$ & $\begin{array}{l}\text { ICD: } \\
\text { - Inj. Conjunctiva corneal abrasion } \\
\text { wo FB }(71,17.8 \%) \\
\text { - Open wound of other parts of } \\
\text { head }(56,14 \%) \\
\text { - Contusion of eyelid and periocular } \\
\text { area }(44,11 \%)\end{array}$ & $\begin{array}{l}\text { ICD: } \\
\text { - Inj. conjunctive corneal abrasion } \\
\text { WO fb }(72,18.6 \%) \\
\text { - Contusion of eyelid and periocular } \\
\text { area }(39,10.1 \%) \\
\text { - Open wound of other parts of } \\
\text { head }(36,9.3 \%)\end{array}$ \\
\hline $\begin{array}{c}\text { Gender: } \\
\text { Male: } 385,19.6 \% \\
\text { Female: } 200,17.3 \%\end{array}$ & $\begin{array}{c}\text { Gender: } \\
\text { Male: } 244,12.4 \% \\
\text { Female: } 155,13.4 \%\end{array}$ & $\begin{array}{c}\text { Gender: } \\
\text { Male: } 266,68.56 \% \\
\text { Female: } 122,31.44 \%\end{array}$ \\
\hline $\begin{array}{l}\text { Type of place: } \\
\text { - Home }(347,59.3 \%) \\
\text { - Primary, secondary school (93, } \\
\text { 15.9\%) } \\
\text { - Unspecified place }(42,7.2 \%)\end{array}$ & $\begin{array}{l}\text { Type of place: } \\
\text { - Home }(193,48.4 \%) \\
\text { - Primary, secondary school (69, } \\
\text { 17.3\%) } \\
\text { - Unspecified place }(31,7.8 \%)\end{array}$ & $\begin{array}{l}\text { Type of place: } \\
\text { - Home (173, } 44.6 \%) \\
\text { - Primary, secondary school (100, } \\
\text { 25.8\%) } \\
\text { - Oval, fields, pitch (43. } 11.1 \%)\end{array}$ \\
\hline $\begin{array}{l}\text { Activity: } \\
\text { - Playing (211,36.1\%) } \\
\text { - Unspecified activity (76, 13\%) } \\
\text { - Engaged in formal education } \\
\text { activity }(71,12.1 \%)\end{array}$ & $\begin{array}{l}\text { Activity: } \\
\text { - Playing }(135,33.8 \%) \\
\text { - Other specified activity }(64,16 \%) \\
\text { - Unspecified activity }(52,13 \%)\end{array}$ & $\begin{array}{l}\text { Activity: } \\
\text { - Playing (128, 33\%) } \\
\text { - Engaged in formal education } \\
\text { activity }(77,19.8 \%) \\
\text { - Unspecified activity }(49,12.6 \%)\end{array}$ \\
\hline $\begin{array}{l}\text { Major injury factor: } \\
\text { - Table, desk, bench, etc. }(97,16.6 \%) \\
\text { - Other or unspecified structure or } \\
\text { fixture }(51,8.7 \%) \\
\text { - Wall }(26,4.4 \%)\end{array}$ & $\begin{array}{l}\text { Major injury factor: } \\
\text { - Ball }(55,13.8 \%) \\
\text { - Bat, racquet, hockey stick, etc. }(49, \\
\text { 12.3\%) } \\
\text { - Other toy }(41,10.3 \%)\end{array}$ & $\begin{array}{l}\text { Major injury factor: } \\
\text { Person }(339,87.4 \%)\end{array}$ \\
\hline $\begin{array}{l}\quad \text { Part of place: } \\
\text { - Unspecified part of place }(162, \\
27.7 \%) \\
\text { - Bedroom }(53,9.1 \%) \\
\text { - Other, interior }(45,7.7 \%)\end{array}$ & $\begin{array}{l}\text { Part of place: } \\
\text { - Other, exterior }(49,12.3 \%) \\
\text { - Garden, park, national park, } \\
\text { backyard }(41,10.3 \%) \\
\text { - Oval/field }(40,10 \%)\end{array}$ & $\begin{array}{l}\text { Part of place: } \\
\text { - Unspecified part of place (177, } \\
\text { 45.6\%) } \\
\text { - Oval/field (47, 12.1\%) } \\
\text { - Garden, park, national park, } \\
\text { backyard (32, 8.2\%) }\end{array}$ \\
\hline $\begin{array}{c}\text { Time: } 3-6 \text { p.m. }(160,27.4 \%) \\
p<0.001\end{array}$ & $\begin{array}{c}\text { Time: } 3-6 \text { p.m. }(139,34.8 \%) \\
p<0.001\end{array}$ & $\begin{array}{c}\text { Time: } 3-6 \text { p.m. }(105,27.1 \%) \\
p<0.001\end{array}$ \\
\hline
\end{tabular}

The first three most common mechanisms of injury within this age group were contact with a person $(275,23 \%)$, contact with a moving object $(235,19.6 \%)$, and contact with a static object $(129,10.8 \%)$. There were a total of 1196 children in this age group within the dataset.

Superficial injury of other parts of head was verified as being the most common ICD for the most common injury mechanism category, followed by superficial injury of head part not otherwise specified and fracture of nasal bones as the second and third most common ICDs. respectively. However, injury conjunctiva corneal abrasion without FB was the most common ICD for the second most common mechanism of injury, followed by contusion of the eyelid and periocular area and open wound of other parts of head, respectively, as the second and third most common injury mechanisms. Superficial injury of head part not otherwise specified and superficial injury of other parts of head were the second and third ICD for the third most common injury mechanism within this age group (Table XLII). Oval/field was the was the main part of place where the children were injured for the first and second most common mechanisms of injury, whereas unspecified part of place was the most common part of place for injuries that occurred in the third mechanism of injury category within this age group. Another person was the most common injury factor for the first mechanism of injury, whereas a ball was the most common injury factor for the second most common mechanism of injury, and other or unspecified structure or fixture was the most common factor for the third most common mechanism of injury in this age group.

The highest frequency of injuries occurred at midday -3 p.m. for the most common mechanism of injury, whereas for the second most common mechanism of injury, the greatest incidence of injury occurred at 9 a.m. - midday. For the third most common mechanism of injury, the highest incidences of injury occurred at 3-6 p.m. (Table XLII). 
Table XLII. Injury characteristics by mechanism of injury in the 10-12-years age group ( $n=1196,11.2 \%)$

\begin{tabular}{|c|c|c|}
\hline $\begin{array}{l}\text { Mechanism of injury } 1 \text { : } \\
\text { Contact with person } \\
(n=275)\end{array}$ & $\begin{array}{l}\text { Mechanism of injury } 2 \text { : } \\
\text { Contact with moving object } \\
\qquad(n=235)\end{array}$ & $\begin{array}{l}\text { Mechanism of injury 3: } \\
\text { Contact with static object } \\
(n=129)\end{array}$ \\
\hline $\begin{array}{l}\text { ICD: } \\
\text { - Superficial injury of other parts of } \\
\text { head }(37,13.5 \%) \\
\text { - Superficial injury of head part no } \\
\text { otherwise specified ( } 34,12.4 \%) \\
\text { - Fracture of nasal bones }(27,9.8 \%) \\
\text { and contusion of eyelid and } \\
\text { periocular area }\end{array}$ & $\begin{array}{l}\text { ICD: } \\
\text { - Injury conjunctiva corneal abrasion } \\
\text { without FB }(36,15.3) \\
\text { - Contusion of eyelid and periocular } \\
\text { area }(25,10.6 \%) \\
\text { - Open wound of other parts of } \\
\text { head }(23,9.8 \%)\end{array}$ & $\begin{array}{l}\text { ICD: } \\
\text { - Open wound of other parts of } \\
\text { head }(28,21.7 \%) \\
\text { - Superficial injury of head part no } \\
\text { otherwise specified }(14,10.9 \%) \\
\text { - Superficial injury of other parts of } \\
\text { head }(14,10.9 \%)\end{array}$ \\
\hline $\begin{array}{c}\text { Gender: } \\
\text { Male: } 213,77.45 \% \\
\text { Female: } 62,22.55 \% \\
\end{array}$ & $\begin{array}{c}\text { Gender: } \\
\text { Male: } 158,67.23 \% \\
\text { Female: } 77,32.77 \% \\
\end{array}$ & $\begin{array}{c}\text { Gender: } \\
\text { Male: } 90,69.77 \% \\
\text { Female: } 39,30.23 \% \\
\end{array}$ \\
\hline $\begin{array}{l}\text { Type of place: } \\
\text { - Oval, field, pitch }(87,31.6 \%) \\
\text { - Primary, secondary school }(72, \\
\text { 26.2\%) } \\
\text { - Home }(69.25 .1 \%)\end{array}$ & $\begin{array}{l}\quad \text { Type of place: } \\
\text { - Home }(72,30.6 \%) \\
\text { - Primary, secondary school (65, } \\
\text { 27.7\%) } \\
\text { - Oval, fields, pitch }(43,18.3 \%)\end{array}$ & $\begin{array}{l}\text { Type of place: } \\
\text { - Home }(60,46.5 \%), \\
\text { - Primary, secondary school (22, } \\
\text { 17.1\%) } \\
\text { - Unspecified place }(10,7.8 \%)\end{array}$ \\
\hline $\begin{array}{l}\text { Activity: } \\
\text { - Football, rugby, soccer (102, } \\
37.1 \%) \text { ) Engaged in formal education ( } 38, \\
\text { - } 13.8 \%) \\
\text { - Playing }(38,13.8 \%)\end{array}$ & $\begin{array}{l}\text { Activity: } \\
\text { - Playing (52, } 22.1 \%) \\
\text { - Ball sport with racquet, bat } \\
\text { (baseball, cricket, hocky) (47, 20\%) } \\
\text { - Engaged in formal education (33, } \\
\text { 14\%) }\end{array}$ & $\begin{array}{l}\text { Activity: } \\
\text { - Playing (37, 28.7\%) } \\
\text { - Other specified activity }(19,14.7 \%) \\
\text { - Resting, sleeping, eating, other } \\
\quad(17,13.2 \%)\end{array}$ \\
\hline $\begin{array}{c}\text { Major injury factor: person (252, } \\
\qquad 91.6 \%)\end{array}$ & $\begin{array}{l}\quad \text { Major injury factor: } \\
\text { - Ball (84, 35.7\%) } \\
\text { - Bat, racquet, hockey stick, etc. ( } 34, \\
\text { 14.5\%) } \\
\text { - Tree (includes branch stick, twig) } \\
\quad(15,6.4 \%)\end{array}$ & $\begin{array}{l}\quad \text { Major injury factor: } \\
\text { - Other or unspecified structure or } \\
\text { fixture }(19,14.7 \%) \\
\text { - Wall }(9,7 \%) \\
\text { - Table, desk, bench, etc. }(19,14.7 \%)\end{array}$ \\
\hline $\begin{array}{l}\text { Part of place: } \\
\text { - Oval/field (85, 30.9\%) } \\
\text { - Unspecified part of place }(74,26.9 \%) \\
\text { - Other, exterior }(34,12.4 \%)\end{array}$ & $\begin{array}{l}\quad \text { Part of place: } \\
\text { - Oval/field }(57,24.3 \%) \\
\text { - Unspecified part of place }(55,23.4 \%) \\
\text { - Other, exterior }(42,17.9 \%)\end{array}$ & $\begin{array}{l}\text { Part of place: } \\
\text { - Unspecified part of place }(36, \\
27.9 \%) \\
\text { - Garden, park, national park, } \\
\text { backyard }(15,11.6 \%) \\
\text { - Other, interior }(912,9.3 \%)\end{array}$ \\
\hline $\begin{array}{c}\text { Time: midday - } 3 \text { p.m. }(96,34.9 \%) \\
p<0.001\end{array}$ & $\begin{array}{c}\text { Time: } 9 \text { a.m. }- \text { midday }(64,27.2 \%) \\
p<0.001\end{array}$ & $\begin{array}{c}\text { Time: } 3-6 \text { p.m. }(36,27.9 \%) \\
p<0.001\end{array}$ \\
\hline
\end{tabular}

\section{Discussion}

The number of injury cases varied among age groups with head and facial injuries, and the mechanism, severity, nature, and type of injury sustained were also different. This might be due to the difference in the activities in which the children were engaged at the time of injury. In addition, the degree of osseous maturity of the developing paediatric structures determine the characteristics and nature of injuries. This results of the present study are consistent with previous reports $[11,19]$. We suggest that injury prevention strategies need to be age and mechanism focused.

Injury literature shows that boys are more likely to sustain injuries than girls. Similarly, our study demonstrated an overrepresentation of males with a male : female ratio of $1.7: 1$. This might be because boys are generally more boisterous, or it could be related to their greater tendency for risk taking and spending more time taking part in activities compared to girls. However, a recent report shows a trend toward a more equal male : female ratio [20]. This changing trend can be attributed to the same activities shared by both genders, although their abilities are different.

We have clearly demonstrated that the young age groups are most likely to sustain head and facial injuries. One- and two-year-olds accounted for one-third of all presentations in this study. This finding may be due to toddlers (1-2 years old) are still learning how to walk and coordinate that movement with their growing bodies without knowing the risks; hence, they get into a bit of trouble and sustain injuries, especially if there are no effective safety measurements or parents' 
attention. Similar studies have reported the same results $[21,22]$.

Furthermore, we have reported the 'typical' injury in each age group. As an example, in the 1-2-years age group, the most common cause of injury is contact with a static object and is likely to have occurred in the home, through contact with a table, bench etc., or a wall, in contrast to a previous report, which reported that falls were the most common injury in the first year of life [23]. This difference is because the aetiological factor varies significantly depending on the situation, as well as geographically and culturally.

Many studies report over-representation of indigenous children in Australian injury statistics $[24,25]$, but because we do not have precisely-defined source populations for our ED data, we were unable to explore this in our study. The justification for indigenous over-representation is due to socioeconomic disadvantage, lack of safety education, and living in remote areas with higher risk of injury $[26,27]$. There can also be issues with identification of indigenous status, most often under-reporting. However, in our study, we found that many injury characteristics varied with Indigenous status, so culturally specific injury prevention messages and approaches may be required.

Time can be an influence on head and facial injuries by variation in the time of day, weekday, and season. Distribution according to time allows us to observe a greater tendency of injury incidence at the end day (3-6 p.m.) when the parents are at their most tired, in particular with working mothers, who might give a low level of attention to their children at that time. This result was similar to previous reports [28, 29].

Furthermore, it has been observed in the present study that the distribution of frequency of paediatric head and facial injuries was proportionally higher on weekends than on weekdays, similarly to previous reports [10]. The reason for this finding is because on weekends there are more opportunities for activities and recreation, and possibly because primary care centres such as GPs are not open.

In addition, we observed in this study that the highest frequency of injuries occurred in autumn. This finding might be due to differences in seasons according to the latitude and longitude of the north part of Queensland, Australia. However, there are no well-defined four seasons in most parts of Australia. In contrast to other reports, the incidence of injury can be changed according to the latitude of the region or country where the study was conducted [10,30].

Injury review shows that paediatric head and facial injuries are constantly changing, and they differ from country to country, as well as in dif- ferent demographic regions in the same country, because of the impact of social, cultural, and economic factors as well as environmental conditions $[10,31]$.

Unlike in adults, where traffic accidents are predominant, falls were reported by previous studies as the leading cause of head and facial injuries in children [32]. In the present study, the main reason for injury was a contact with a static object, followed by cutting, tearing, and other falls. This shows that aetiological factors vary significantly depending on the different situations.

According to the severity of head and facial injuries, it has been observed that the majority of cases needed semi-urgent intervention (60 min), followed by urgent triage (30 $\mathrm{min}$ ). This finding depended on the mechanism of head facial injuries that was the most influential factor in the severity of injuries. This finding was in accordance with other studies $[11,20]$.

In the present study, home (indoor) was the most common place of occurrence of injuries to children, followed by garden park and national park (outdoor). In addition, it has been found that the vast majority of paediatric injuries occurred during leisure activity in young age, whereas sport activities-related injuries occurred in older age groups. Furthermore, playing injuries were predominant among all types of activities. This may reflect the difference in the trend of injuries between young and old age groups. Thus, understanding of these injuries and their environment is key for the clinician's role in injury prevention and education. This finding was similar to previous studies [5, 11, 29].

According to the body region variable, the vast majority of head and facial injuries among paediatric age groups were unintentional injuries. It was observed that the face (excludes eye) was more predominant compared to the head. This finding might be because the face is the most exposed unprotected area in the body, as well as being the most sensitive part to injury. However, literature review shows that the injury aetiology is an influential factor in determining the body region involved, as well as the severity of injury $[33,34]$.

\section{Recommendations}

Significant elements and aspects need to be highlighted to improve the epidemiological model of paediatric head and facial injuries in Queensland, Australia, as follows:

- Regarding head and facial injury prevention, knowing the epidemiology profile of children involved in head and face injuries allows us to monitor and control certain risk factors that would prevent or lessen the incidence of such 
injuries [10]. Additionally, insight into the epidemiology of head and facial injuries is useful not only in prevention strategies, but also in decision-making for patient care, development of optimal treatment regimens, and appropriate resource allocation [35]. For this reason, hospital records serve the purpose of collecting key information on incidences and consequences [29]. Furthermore, studies showed that prevention programs play many roles in helping to reduce the burden of injury [36, 37].

- Thus, preventive measures should be focused on reducing the incidence and/or minimising the severity of injury. The morbidity of face and head injuries in children can be reduced significantly under instruction and enforcement of safety measures.

- Teaching the public the importance of effective means to minimise the incidence of injury [38]

- Public health specialists should provide professional advice and make suggestions to parents, teachers, and caregivers [39].

- Children should be encouraged to develop healthy habits at an early age (e.g. wearing a helmet and a seat belt), and children in the growing phase should be monitored periodically [5].

- Medical staff could establish databases of paediatric injuries to corroborate high-frequency categories of injury and verify the effectiveness of developed prevention programs. Outcome assessment of such databases in multidisciplinary morbidity and mortality conferences could lead to improved patient care and better-educated health providers [40].

\section{Limitations of the study}

The QISU database is not truly representative of EDs and their source populations within Queensland. QISU data captures about $25 \%$ of all injury-related ED presentations. However, according to the published paper of Siskind and Scott [23], the results can be considered to be representative in general terms of childhood injuries and the circumstances surrounding them.

Additionally, the researcher had no control over data extraction from the QISU database, so they cannot guarantee the appropriateness, completeness, or specificity of search terms. Furthermore, the injury surveillance data that contributes to the QISU database is collected in busy EDs. Some data may be incomplete or coded inaccurately.

\section{Conclusions}

The results of this retrospective study provide important data for the design of future plans for the prevention of paediatric head and facial injuries. We have demonstrated that the mechanism of injury varies with age, so we suggest that in jury-prevention strategies should be age and mechanism focused. Elements need to be highlighted to improve the epidemiological model of paediatric head and facial injuries in Queensland, Australia. Overall, we hope this work contributes to a decrease in the incidence and severity of paediatric head and facial injuries in Queensland, Australia.

\section{Conflict of interest}

The author declares no conflict of interest.

\section{References}

1. Abosadegh M, Rahman S. Epidemiology and incidence of traumatic head injury associated with maxillofacial fractures: a global perspective. J Int Oral Health 2018; 10: 63-70.

2. Injury Epidemiology: Research and Control Strategies. ed 2. Robertson LS. Ann Emergency Med 1999; 34: 305-6.

3. Lynham A, Tuckett J, Warnke P. Maxillofacial trauma. Austral Fam Phys 2012; 41: 172-82.

4. Robertson LS. Injury Epidemiology: Research and Control Strategies. $3^{\text {rd }}$. Oxford University Press, New York 2007.

5. Qing-Bin ZMDDDS, Zhao-Qiang ZMD, Dan CMDP, Yan ZMD. Epidemiology of maxillofacial injury in children under 15 years of age in southern China. Oral Surg Oral Med Oral Pathol Oral Radiol 2013; 115: 436-41.

6. Singh V, Malkunje L, Mohammad S, Singh N, Dhasmana S, Das SK. The maxillofacial injuries: a study. Natl J Maxillofac Surg 2012; 3: 166-71.

7. Shahim FN, Cameron P, McNeil JJ. Maxillofacial trauma in major trauma patients. Austral Dental J 2006; 51: 225-30.

8. Krausz AA, Krausz MM, Picetti E. Maxillofacial and neck trauma: a damage control approach. World J Emerg Surg 2015; 10: 31.

9. Ajagbe HA, Daramola JO. Pattern of facial bone fractures seen at the University College Hospital, Ibadan, Nigeria. East African Med J 1980; 57: 267-73.

10. Chrcanovic BR. Factors influencing the incidence of maxillofacial fractures. Oral Maxillofac Surg 2012; 16: 3-17.

11. Kambalimath HV, Agarwal SM, Kambalimath DH, Singh M, Jain N, Michael P. Maxillofacial injuries in children: a 10 year retrospective study. J Maxillofac Oral Surg 2013; 12: 140-4.

12. Boyette JRMD. Facial fractures in children. Otolaryngol Clin N Am 2014; 47: 747-61.

13. Muñante-Cárdenas JL, Olate S, Asprino L, De Albergaria Barbosa JR, De Moraes M, Moreira RWF. Pattern and treatment of facial trauma in pediatric and adolescent patients. J Craniofac Surg 2011; 22: 1251-5.

14. Mijiti A, Ling W, Tuerdi M, et al. Epidemiological analysis of maxillofacial fractures treated at a university hospital, Xinjiang, China: a 5-year retrospective study. J Craniomaxillofac Surg 2013; 42: 227-33.

15. Lee K. Global trends in maxillofacial fractures. Cranial Maxillofac Trauma Reconstruction 2012; 5: 213-22. 
16. Björklund K, Liski A, Samposalo $H$, et al. "Together at school" - a school-based intervention program to promote socio-emotional skills and mental health in children: study protocol for a cluster randomized controlled trial. BMC Public Health 2014; 14: 1042.

17. Krug EG, Sharma GK, Lozano R. The global burden of injuries. Am J Public Health 2000; 90: 523-6.

18. Moll EK, Donoghue AJ, Alpern ER, Kleppel J, Durbin DR, Winston FK. Child bicyclist injuries: are we obtaining enough information in the emergency department chart? Injury Prevention 2002; 8: 165-9.

19. Cassar-Pullicino VN, Leone A. Imaging in paediatric spinal injury. Trauma 2017; 19 (1 Suppl): 50-74.

20. Gassner R, Tuli T, Hächl O, Rudisch A, Ulmer H. Cranio-maxillofacial trauma: a 10 year review of 9543 cases with 21067 injuries. J Craniomaxillofac Surg 2003; 31: 51-61.

21. Jagnoor J, Bassani DG, Keay L, et al. Unintentional injury deaths among children younger than 5 years of age in India: a nationally representative study. Injury Prevention 2011; 17: 151-5.

22. Oyetunji TA, Stevenson AA, Oyetunji AO, et al. Profiling the ethnic characteristics of domestic injuries in children younger than age 5 years. Am Surgeon 2012; 78: 426-31.

23. Siskind V, Scott D. Injuries leading to hospitalisation in the first year of life: analysis by trimester of age using coded data and textual description. Austral N Zeal J Public Health 2013; 37: 168-72.

24. Margeson A, Gray S, Alyssa M, Selena G. Interventions aimed at the prevention of childhood injuries in the indigenous populations in Canada, Australia and New Zealand in the last 20 years: a systematic review. Int J Environm Res Public Health 2017; 14: 589.

25. Möller H, Harvey L, Falster K, Ivers R, Clapham KF, Jorm L. Indigenous and non-Indigenous Australian children hospitalised for burn injuries: a population data linkage study. Med J Australia 2017; 206: 392-7.

26. Pediatrics; Data on Pediatrics Reported by Researchers at Simon Fraser University (Disparities in Paediatric Injury Mortality between Aboriginal and Non-Aboriginal Populations in British Columbia, 2001-2009). Pediatrics Week 2016

27. Möller H, Falster K, Ivers R, et al. Inequalities in hospitalized unintentional injury between aboriginal and non-aboriginal children in New South Wales, Australia. Am J Public Health 2016; 106: 899-905.

28. Bremberg S. Health promotion at Swedish Child Health Centres. Acta Paediatr 2000; 89 (Suppl 434): 53-6.

29. Ferraz-Torres M, Belzunegui-Otano T, Martínez-García O, Iriarte-Cerdán L, Salgado-Reguero E. Epidemiological characteristics and overall burden of accidental injuries in Navarra, Spain: epidemiology of injuries in children. J Trauma Nursing 2016; 23: 231-6.

30. Chrcanovic BR, Freire-Maia B, Souza LN, Araújo VO, Abreu MHNG. Facial fractures: a 1-year retrospective study in a hospital in Belo Horizonte. Brazil Oral Res 2004; 18: 322-8.

31. Chan KH, Gao D, Bronsert M, Chevallier KM, Perkins JN. Pediatric facial fractures: demographic determinants influencing clinical outcomes. Laryngoscope 2016; 126: 485-90.

32. Qudah MA, Al-Khateeb T, Bataineh AB, Rawashdeh MA. Mandibular fractures in Jordanians: a comparative study between young and adult patients. J Craniomaxillofac Surg 2005; 33: 103-6.
33. Abosadegh MM, Rahman SA, Saddki N. Association of traumatic head injuries and maxillofacial fractures: a retrospective study. Dental Traumatol 2017; 33: 369-74.

34. Rallis GDDSMDP, Stathopoulos PDDSMDP, Igoumenakis DDDSMD, Krasadakis CDDSMD, Mourouzis CDDSMDP, Mezitis MDDS. Treating maxillofacial trauma for over half a century: how can we interpret the changing patterns in etiology and management? Oral Surg Oral Med Oral Pathol Oral Radiol 2015; 119: 614-8.

35. Hogg NJV, Stewart TC, Armstrong JEA, Girotti MJ. Epidemiology of maxillofacial injuries at trauma hospitals in Ontario, Canada, between 1992 and 1997. J Trauma Injury Infect Critical Care 2000; 49: 425-32.

36. Rivara FP. Prevention of injuries to children and adolescents. Injury Prevention 2002; 8: iv5-8.

37. Rogmans WHJ. Joint action on monitoring injuries in Europe (JAMIE). Arch Public Health 2012; 70: 19.

38. Chandra Shekar B, Reddy C. A five-year retrospective statistical analysis of maxillofacial injuries in patients admitted and treated at two hospitals of Mysore city. Indian J Dental Res 2008; 19: 304-8.

39. Arbogast KB, Durbin DR, Kallan MJ, Menon RA, Lincoln AE, Winston FK. The role of restraint and seat position in pediatric facial fractures. J Trauma Injury Infect Crit Care 2002; 52: 693-8.

40. Powell EC, Tanz RR. Cycling injuries treated in emergency departments: need for bicycle helmets among preschoolers. Arch Pediatr Adolescent Med 2000; 154: 1096-100. 